\title{
The Effect of Finite Temperature on the Jamming Transition
}

\author{
Dissertation zur Erlangung des \\ mathematisch-naturwissenschaftlichen Doktorgrades \\ "Doctor rerum naturalium" der \\ Georg-August-Universität Göttingen
}

im Promotionsprogramm der International Max Planck Research School "Physics of Biological and Complex Systems" der Göttingen

Graduate School for Neurosciences, Biophysics, and Molecular Biosciences

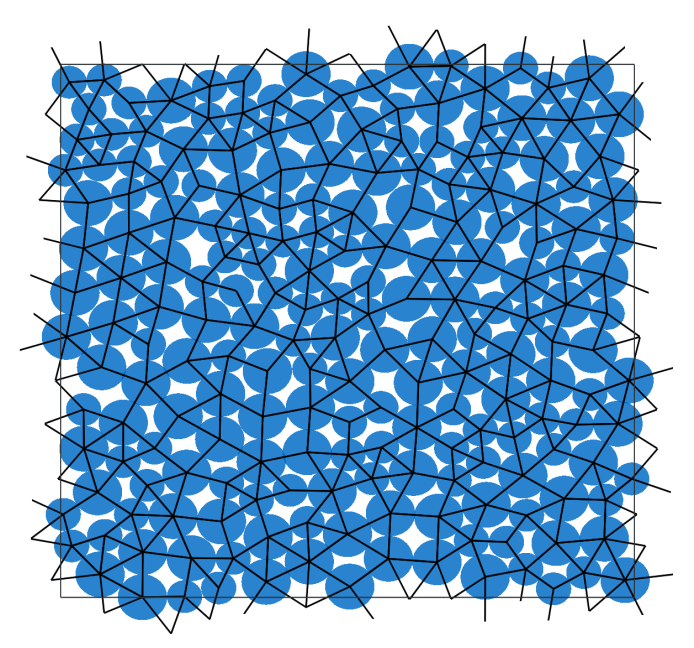

vorgelegt von

Clemens N. Buß

aus Heidelberg

Göttingen, 2016 



\section{Examination board}

Prof. Dr. Oskar Hallatschek, referee, main supervisor and member of the thesis committee.

Biological Physics and Evolutionary Dynamics Group

University of California, Berkeley

Prof. Dr. Florentin Wörgötter, referee and member of the thesis committee.

Computational Neuroscience

Georg-August-Universität Göttingen

Dr. Claus Heussinger, second supervisor and member of the thesis committee.

Institute for Theoretical Physics

Georg-August-Universität Göttingen

\section{Prof. Dr. Stephan Herminghaus}

Dynamics of Complex Fluids

Max Planck Institute for Dynamics and Self-Organization

\section{Prof. Dr. Marc Timme}

Network Dynamics

Max Planck Institute for Dynamics and Self-Organization

Prof. Dr. Marcus Müller

Computational Soft Condensed Matter

Georg-August-Universität Göttingen

Oral examination: 19. June 2015 
I confirm that I have written this thesis independently and with no other sources and aids than quoted.

Göttingen, 2016 


\section{Abstract}

We study the effect of finite temperatures on spring networks obtained from amorphous, jammed packings of repulsive spheres. By means of a Monte Carlo Metropolis scheme, we analyse this two-dimensional model of an amorphous solid with fixed connectivity. Those spring networks act as a general model system for the thermal behaviour of amorphous solid materials. A particularly interesting feature of the model system is that it contracts with increasing temperature and starts to collapse when crossing a certain threshold temperature.

Our observables include thermal expansion, bulk and shear modulus and the localisation length of particles in response to changes in temperature, pressure and distance from the isostatic point. We find scaling relations which strongly support theoretical considerations of the model system based on the analysis of its soft modes and their non-affine contributions.

Using these results we are able to construct the phase diagram for the system so that we can understand the parameters which lead to collapse.

In order to complement the research on spring networks, we investigate the processes in jammed packings. One would expect that these packings expand with increasing temperature. Counterintuitively, the packings contract similarly to the spring networks in a regime of low temperatures and far away from isostaticity. Based on this evidence we conjecture that spring networks might be seen as a higher level of abstraction with regard to the corresponding sphere packings. 



\section{Contents}

Abstract $\quad 5$

$\begin{array}{ll}\text { Contents } & 7\end{array}$

$\begin{array}{llr}1 & \text { Introduction } & 9\end{array}$

1.1 Jamming. . . . . . . . . . . . . . . . . . . 9

1.2 Glasses.............................. 21

1.3 Negative Thermal Expansion . . . . . . . . . . . . . . 24

1.4 Structure of this work . . . . . . . . . . . . . 28

2 Generating jammed sphere packings 31

2.1 Measuring coordination . . . . . . . . . . . . 35

2.2 Identification of the critical coordination number . . . . . . . . 36

2.3 Pruning protocol $\ldots \ldots \ldots 37$

$\begin{array}{lll}3 & \text { Model } & 39\end{array}$

4 Methods 43

4.1 A small remark on the role of simulations . . . . . . . . . . . . 43

4.2 Monte Carlo . . . . . . . . . . . . . . . . . . 44

4.3 Implementation . . . . . . . . . . . . . . . . . . . . . 47

4.4 Concluding remarks . . . . . . . . . . . . . . . . 68

5 Thermally Activated Networks of Springs $\quad 71$

5.1 Area development . . . . . . . . . . . . . . 72 
CONTENTS

5.2 Bulk modulus . . . . . . . . . . . . . . . . . . . . . 83

5.3 Shear modulus . . . . . . . . . . . . . . . . . 9 90

5.4 Localisation length . . . . . . . . . . . . . . . . . 92

6 Dependence on network structure $\quad 97$

$\begin{array}{lll}7 & \text { Phase diagram of jammed spring networks } & 101\end{array}$

8 Jammed Packings of Spheres 105

8.1 Pressure development in NVT . . . . . . . . . . . . 105

8.2 Breaking of contacts . . . . . . . . . . . . . 109

8.3 Brief note: the handling of rattlers . . . . . . . . . . . . 111

8.4 Bulk modulus . . . . . . . . . . . . . . . . . . . . . . . 112

8.5 Localisation length . . . . . . . . . . . . . . . . . 114

9 Discussion $\quad 115$

9.1 Spring networks show negative thermal expansion . . . . . . . 115

9.2 Spring networks exhibit a finite bulk modulus above the jamming point which softens with increasing temperature . . . . . . . 115

9.3 The shear modulus of spring networks stiffens with temperature . 116

9.4 The characteristics of spring networks are directly connected to their anomalous modes . . . . . . . . . . . . . . 116

9.5 Negative thermal expansion is a feature of most spring networks . 116

9.6 The structure of spring networks strongly affects their mechanical features . . . . . . . . . . . . . . . 117

9.7 Jammed spring networks expose a contraction-collapse phase transition with strong indication of a critical point . . . . . . . . . 118

9.8 Spring networks contract, jammed packings do not always expand 118

9.9 Spring networks help in understanding thermalized jammed packings 119 


\section{Introduction}

In the following, we will give a short introduction to jamming because the concept is constitutive to the work presented in this thesis. Having introduced jamming, we will give an overview on glasses and the glass transition as well as the phenomenon of negative thermal expansion.

\subsection{Jamming}

Granular materials or fragile matter such as sand exhibit a surprising and fascinating property: they jam. Jamming occurs with certain materials at the transition from a flowing state to a rigid one. Increasing compression or lowering the applied shear stress induces this transition by bringing the constituents of the material into contact. At a certain point the constituents do not have the possibility of rearranging and get stuck [1]. In this way they become solid. The rigid state of the material is characterized by its elastic properties such as a finite bulk and shear modulus [2]. The jamming transition is exciting because both the rigid and the flowing state are disordered in their microscopic structure. Further examples of materials and phenomena to which the theory applies are for instance foams, such as shaving foam, toothpaste, mayonnaise and all types of granular media like cereals and coffee beans among others as well as bacteria in a crack or crowds of panicking people trying to pass a bottleneck [3]. Figure 1.1 shows some of these examples.

Jamming is a path to rigidity different from a common thermodynamic or chemical first order phase transition [5]. Usually matter goes from liquid to solid involving the emission of latent heat [6]. This solid-fluid transition is driven by temperature, and one speaks of a change from one thermodynamic ground state to another. The liquid phase is governed by unordered motions while the solid phase is usually highly ordered and the dynamics are constrained because the constituents form chemical bonds. In the jamming transition, in contrast, the dynamics of the constituting particles get more and more constrained until they get stuck. They are trapped in a small region of phase space. This marks the flowing-rigid transition in jamming. How particles get stuck will be discussed in greater detail in Section 1.1.1. The jammed material can be made to flow again 


\section{Introduction}

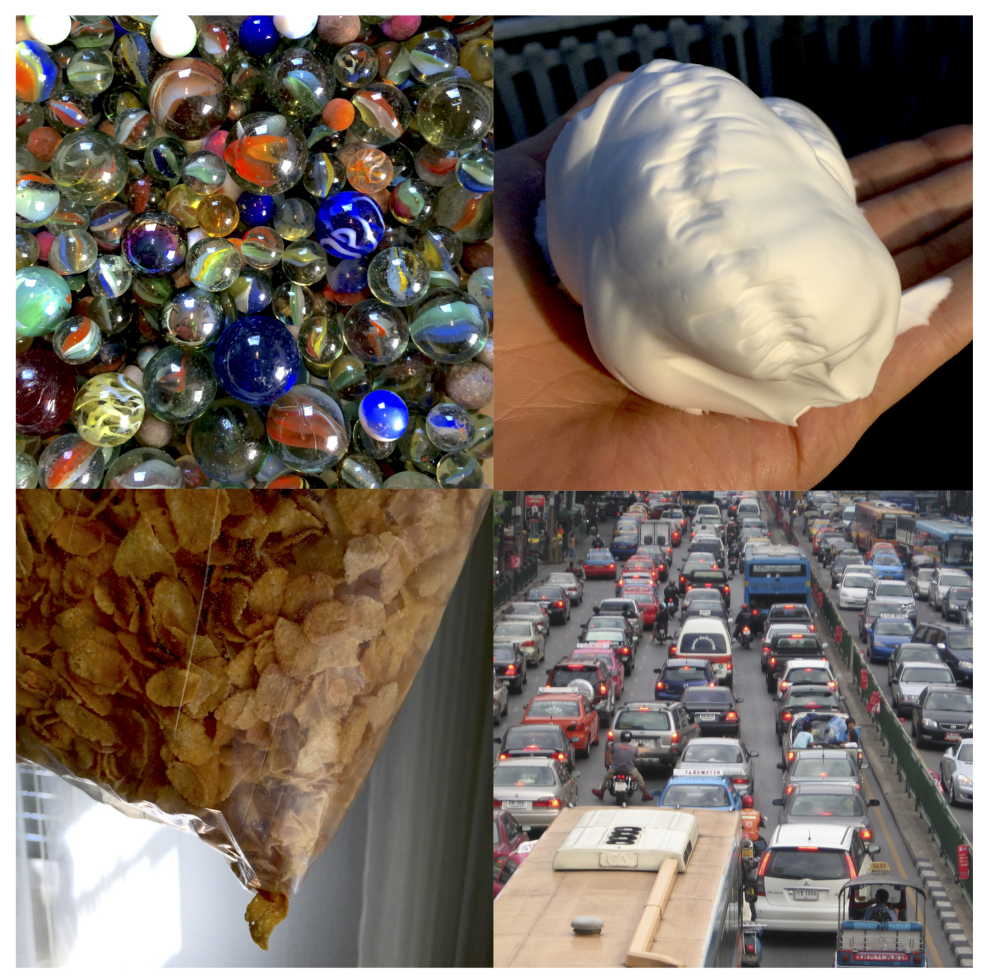

Figure 1.1: Examples of jamming in everyday life. Top left: marbles in a box representing a packing of hard spheres. By pure accident the marbles are of glass themselves. Top right: shaving foam maintaining a static structure. Bottom left: cereals stuck in a bag. Even though a single corn flake would fit through the gap nothing flows out of the bag. Shaking - a mesoscopic temperature - can make this jammed systems flow again. Bottom right: cars stuck in a traffic jam [4] in Bangkok (Photo from wikipedia.org, CC BY 2.0).

by increasing stress in the case of foams or by reducing the system's pressure in the case of colloidal suspensions [7,8].

The resulting rigid structure is disordered. It is impossible to tell from the order of the material if it is in the jammed or flowing regime. This particular difference comes up when we compare the microscopic picture close to the jamming transition in the flowing phase to the one in the freshly jammed system. This is in large contrast to the chemical solid-fluid transition where the solid phase is highly ordered and easily discernible. Moreover, the jamming transition does not involve emission of latent heat.

Let us first deal with compression that governs the jamming transition. Together with the effect of shear stress this is what has been mainly investigated in the 
field looking into to many different types of jammed systems $[9,10]$ It is the aim of this work to shed further light on the influence of temperature $[11,12]$.

\subsubsection{Frictionless Soft Spheres}

Historically speaking, jamming describes the athermal, zero-load phenomena of granular materials [5]. Sometimes phenomena induced by load or temperature are lumped into the term as well and we will consider these cases in Section 1.1.6. Yet, at this point we will stick with the historical and more strict definition.

Jamming is conceptually most easily understood and established by the model of soft frictionless spheres which interact repulsively on contact, i.e. through finiteranged forces [13]. This is the case in a two-dimensional box with a fixed area which is filled with round particles and where thermal fluctuations are turned off. Here softness is approximated by the possibility of spheres to overlap, which involves repulsion, but does not cause deformation. Imagine a two-dimensional volume with area $A$ that is filled with a number $N_{1}$ of circles of radius $r_{1}$ and a number $N_{2}$ of circles with radius $r_{2}$. We fill the volume bi-disperse-with particles of two different sizes - to avoid crystallization in the process. This allows us to define the packing fraction or gas fraction $\phi$ of the system as the ratio of the "filled" area over the total area:

$$
\phi=\frac{\pi N_{1} r_{1}^{2}+\pi N_{2} r_{2}^{2}}{A}
$$

$N$ is the total number of spheres $N=N_{1}+N_{2}$, with usually $N_{1}=N_{2}$. The interaction potential $V$ is like a one-sided spring between touching spheres with the exact definition:

$$
V_{i j}= \begin{cases}0, & \text { if } d_{i j} \geq R \\ \frac{1}{\epsilon}\left(R-d_{i j}\right)^{\epsilon}, & \text { if } d_{i j} \leq R .\end{cases}
$$

The distance $d_{i j}$ is measured between the centres of two spheres $i, j$ and $R$ is the sum of their radii. These spheres interact with a potential characterized by the exponent $\epsilon$. In this work we will mostly choose it to be harmonic, i.e. $\epsilon=2$. 


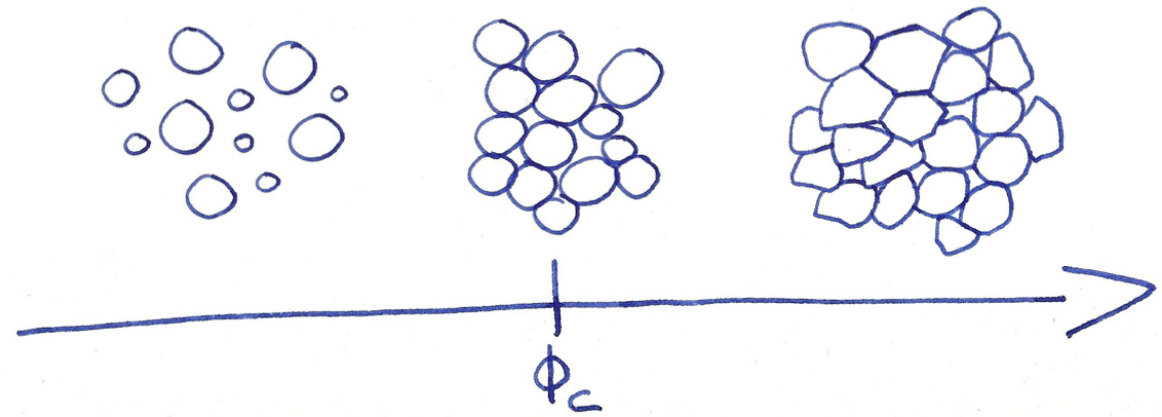

Figure 1.2: Sketch of the route to jamming with the model of soft spheres. Initially, the spheres are loose and distributed in the volume. By increasing their size they start to touch at a certain packing fraction $\phi_{c}$. For values of $\phi \geq \phi_{c}$ the particles are constrained. They are jammed. Further increasing the packing fraction $\phi$ leads to their deformation and increase in system pressure.

\subsubsection{The Jamming Transition}

The section will describe the path to jamming by means of soft spheres in a twodimensional volume. Starting from an initially loose and random configuration of spheres, this system gets jammed by increasing the packing fraction.

Initially, we choose the packing fraction low enough so that we can randomly distribute the spheres in the volume without touching each other. The pressure $p$ is defined via a virial expansion as follows:

$$
p=\frac{\sum_{i j}^{N} \overrightarrow{x_{i j}} \cdot \overrightarrow{f_{i j}}}{2 \cdot A} .
$$

The virial includes all interactions between overlapping particles, where $\overrightarrow{x_{i j}}$ is the position vector between particle $i$ and $j$ and $\overrightarrow{f_{i j}}$ the force between them. The factor 2 can be attributed to the dimensionality of the system. Contributions to the pressure by pushes against the system's wall are zero because the system is athermal. The force is computed from the potential by differentiation with respect to position:

$$
f_{i j}= \begin{cases}0, & \text { if } d_{i j} \geq R \\ \left(R-d_{i j}\right)^{\epsilon-1}, & \text { if } d_{i j} \leq R .\end{cases}
$$


At the beginning the pressure in the system is zero. In the following, we increase the packing fraction by inflating the radii of the spheres and keeping the volume constant. Whenever there is an overlap between particles they can push each other apart so that all forces are relieved. The system is still in the fluid phase. At some point the spheres touch and overlap without a chance to relax all of the forces [14]. This is when they get stuck in the jammed, rigid regime. The spheres form a force network and we can measure a finite pressure $p$. All spheres will contribute to the system pressure, except a few which are not integrated in the jammed state. These spheres which are in no contact with other spheres and thus do not contribute to the overall pressure, are called rattlers [1]. It is safe to say that they amount to less than $5 \%$ of the spheres in the system; this will be discussed in greater detail in section 2 .

The packing fraction for which the packing starts to exhibit a finite pressure is the critical packing fraction $\phi_{c}[15]$. The jamming transition happens at $\phi_{c}$. The exact numeric value of $\phi_{c}$ depends on the finite size of the simulation system [16]. Additionally, it varies between realizations - or in other words the history - of a system of given size. Yet, for a particular realization at hand it is a sharply defined value. Many phenomena in jamming are governed by the distance of the system's packing fraction from $\phi_{c}$.

In contrast to unjammed systems $\phi<\phi_{c}$, jammed systems $\phi>\phi_{c}$ exhibit measurable mechanical moduli. This discriminates the two states of the system mesoscopically. In other words, for $\phi<\phi_{c}$ we can shear and compress the flowing system freely, much like a liquid. For $\phi>\phi_{c}$, the system reacts elastically within certain limits and exhibits finite values for bulk and shear modulus, as we would expect from a solid body. At $\phi=\phi_{c}$ it is exactly at the jamming point $J$ [17]. This is the first moment when the system has a finite total number of contacts $Z$, which is the number of overlapping and thus interacting spheres in the volume. We define the average number of contacts per sphere, the coordination number:

$$
z=\frac{2 Z}{N}
$$

This definition is fully sufficient for a first understanding of the coordination number [13]. The full definition, however can be found in section 2.1.

Increasing the packing fraction further from $\phi_{c}$, we observe that the pressure rises due to an increasing overlap and that the number of contacts per particle increases as well [7]. This has also been shown in experiments [18]. In a foam the overlap can be pictured as deformation of the bubbles [19]. 


\section{Introduction}

\subsubsection{Isostatic Point}

The average coordination number $z$ is an important quantity for understanding the stability of jammed systems. It is also a fundamental parameter for the spring networks we are considering later. We want to know the coordination number $z_{c}$ of the described packing at the jamming point $J$.

To do so this it is important to understand the isostatic point $z_{i s o}$ [1], defined as the state at which a mechanical structure has exactly the minimal number of constraints needed to balance the forces in the system. Through counting arguments we can determine the isostatic point of a system by its static properties. A coordination number $z>z_{i s o}$ is a necessary condition for jamming. As we will see in the case of frictionless soft spheres, they are identical where the system jams, i.e.

$$
z_{c}=z_{i s o}
$$

First of all, we know that forces balance in the volume filled with frictionless soft spheres. If they were not balanced, there would be movement, possibly rearrangement, in the system until it balances. We have $Z=\frac{N z}{2}$ degrees of freedom for the contact forces to balance each of the $N$ spheres in $d$ dimensions. This can only work if:

$$
\frac{N z}{2} \geq d N \Rightarrow z_{c} \geq 2 d
$$

This is in essence the long-known Maxwell criterion for mechanical stability established by James Clerk Maxwell in the 19th century [20]. The minimum value for force balance is the isostatic point, which in our case has a coordination number of $z_{\text {iso }}=2 d$. Thus:

$$
z_{c} \geq z_{\text {iso }}=2 d \text {. }
$$

Secondly, we know that at the critical point our spheres are undeformed but do touch as they have to hold each other in position over the whole system. If they did not touch, the system would be below the critical packing fraction and below the isostatic point. In fact, the coordination number of the system would be zero in that case. If they were deformed we would be beyond the isostatic point in the static regime. Thus, at $z_{c}$ we have $N d$ positional degrees of freedom for the system of spheres and $\frac{N z}{2}$ constraints on every contact for just touching. This can only work if: 


$$
\frac{N z}{2} \leq d N \Rightarrow z_{c} \leq 2 d
$$

(1.7) and (1.9) can both only hold for:

$$
z_{c}=2 d=z_{i s o}
$$

This result can be generalized to higher dimensions, not only spheres, as long as the particles are round and interact without friction. It is agnostic towards the chosen type of repulsive interaction potential as well as towards other polydisperse configurations. The distance to the critical coordination number $\Delta z$ is a parameter better adapted to capture the network structure of a system and its mechanical stability,

$$
\Delta z=z_{c}-z
$$

Yet, due to effects stemming from the finite size of real systems - known as finite size effects [16] - this generally means isostatic values smaller than 4.0, which is the isostatic value for infinite systems as derived in (1.10). The isostatic value of a specific simulated system is referred to as $z_{0}$. In this case $\Delta z$ means:

$$
\Delta z=z_{0}-z .
$$

In most cases this will not lead to misunderstandings. The coordination number is connected with the packing fraction of the system in an interesting non-trivial way:

$$
\Delta z \propto \sqrt{\Delta \phi}
$$

This is the well-known square root scaling of $\mathrm{z}$ with $\Delta \phi=\phi-\phi_{c}$ which holds still for orders of magnitude away from the critical point. We accept it here as a numerical result [17]. How we obtain these results via simulations and how $z_{0}$ is determined will be shown and discussed in detail in 2.2. Introducing friction [21] or different shapes of particles like ellipses [22], spheroids, ellipsoids or spherocylinders, the counting arguments can only deliver a range of possible values for $z_{c}$ [1]. We will not cover these cases here but refer to further reading on.

\subsubsection{Non-Affinity}

A central issue is to understand the processes in a jammed system. As a first model assumption we consider our jammed system as being ordered and derive 


\section{Introduction}

results from that. Loosening the requirement on orderliness we still assume that global deformations scale down linearly to local deformations. If this assumption holds we call our system affine. The assumption of affinity, together with the assumption that we can get macroscopic observables for our system by coarsegraining it over microscopic contacts, defines the effective medium theory [1]. As an example take a packing of spheres in a hexagonal lattice, also known as liquid honeycomb [23]. This system is anticipated to be affine and it has a packing fraction $\phi=\frac{\pi}{2 \sqrt{3}}$ where the contacts and therefore rigidity is lost. However, for $\phi>\phi_{c}$ due to the orderliness the contact number stays constant at 6 independently of $\phi$. Other ordered packings will expose analogue behaviour and thus cannot account for the crucial square root scaling of the coordination number of (1.13).

The elastic response of jammed packings is very different from predictions of effective medium theory. Since effective medium theory neither incorporates isostaticity nor considers local packing specifics it predicts finite shear and bulk moduli below the isostatic point. Yet, packings expose a vanishing shear modulus and a discontinuous drop of the bulk (or compression) modulus [2]. So the loss of order in amorphous matter comes with consequences: the non-affine contributions in jammed packings have to be considered - as they are not negligible - in order to account for the observed phenomena. It is not enough to resort to models of ordered, affine systems. Consequently, because the systems are difficult to handle analytically in every respect, computer simulations are a powerful tool to investigate the effects of the non-affine contributions.

\subsubsection{Vibrational Spectrum}

Jammed systems and customary, crystalline solids show intriguing differences in their vibrational spectrum, too. The textbook example for determining the density of vibrational states is a body with evenly distributed vibrating atoms. The density of state is the relative number of vibrations for a given frequency. For such a crystalline body, following Debye's arguments, we can determine $D(\omega) \propto$ $\omega^{d-1}$ as the scaling for the density of vibrational modes [24] for a frequency $\omega$ and $d$ denoting the dimensionality of the system. These vibrational modes come as transversal or longitudinal plane waves and are often called acoustic modes or sound modes.

Jammed packings do not follow the described Debye-behaviour for the whole frequency spectrum. In fact, they exhibit an excess of low-frequency modes which is not seen in usual solids and cannot be accounted for with Debye's arguments. The closer the system is to the jamming transition, the more extended towards low frequencies the regime of these modes is. As a matter of fact, there is a plateau of low-frequency modes to a crossover frequency $w^{*}$, while below that frequency the 
Debye-behaviour of common solids is found. The crossover frequency $w^{*}$ shifts to lower frequencies, the closer we get to the critical packing fraction, or in other words the smaller we tune $\Delta \phi[25]$.

Silbert et al. [25] found that the characteristic frequency scales like the distance in coordination to the jamming point:

$$
w^{*} \sim \Delta z
$$

Thus, moving a packing towards $J$ it appears less and less like a usual solid body. It does not matter how $J$ is approached, e.g. by lowering pressure.

This peculiar property of the jammed materials is explained by vibrational modes which are known as soft modes or floppy modes. They form the excess of modes compared to normal solids. The terms soft and floppy themselves suggest the fact that these modes require very little to no energy. Below isostaticity zero-energy movements are possible without violating the condition that particles just touch (1.9) and balance their forces (1.7). In this sense a system at the jamming point $J$ is considered marginally stable. Thus, removing a few contacts results in opening up new, generally global, degrees of freedom for the spheres [26].

Consider cutting a square patch of size $l$ out of a packing which is slightly overcoordinated compared to isostaticity. The absolute number of missing contacts at the boundary will be of the order $l$ in two dimensions. If the total number of contacts $Z_{\text {patch }} \approx \Delta z \cdot l^{2}$ in the patch is below the isostatic value, we have created a patch with floppy modes. The number $N_{\text {soft }}$ of these modes can be quantified by substracting the cut contacts at perimeter of dimension $d$ from the number of contacts in the patch [8]:

$$
N_{\text {soft }} \sim l^{d-1}-\Delta z l^{d}
$$

We identify the critical cutting length $l^{*}$ below which the system behaves isostatically and above it like a normal solid. For this we simply check when $N_{\text {soft }}$ is zero and arrive at:

$$
l^{*} \propto \frac{1}{\Delta z}
$$

This scaling relation condenses important properties of jammed packings. Below $l^{*}$ the packing behaves isostatically. Above $l^{*}$, for packings far away from the jamming transition, modes are localized and plane waves. This is consistent with the predictions derived from Debye's arguments for a continuous elastic medium. Thus, the smaller we make $\Delta z$ - the closer we get to the jamming point - the more extended the soft modes become. Modes may extend over the whole system to 


\section{Introduction}

its borders and they can be excited thermally or by shear. In fact, they are much more sensitive to the applied stress than the conventional acoustic modes [8].

Hence, the responses to a force at some point in the packing lead to a highly non-local response which may spread out over the whole system [27]. Yet, note that even far away from the jamming point we can still build a sub-system with a length $l$ smaller than $l^{*} \propto 1 / \Delta z$, which is not rigid. Thus, at short length scales the system behaves isostatically.

In this sub-system so-called anomalous modes appear, which are essentially trial soft modes which disappear at the borders where the sub-system has been cut. They appear above a frequency $\omega^{*} \propto 1 / l^{*}$, consistent with the scalings for the cutting length $l^{*}(1.16)$ and the characteristic frequency $w^{*}(1.14)$. The closer we get to $\omega^{*}$ the more extended anomalous modes become, while the further we move to higher frequencies the more localized they become. At some frequency $\omega$ far above $\omega^{*}$ modes are highly localized. The frequency being further increased the density of states vanishes abruptly. Below, for $\omega<\omega^{*}$, the system behaves like a normal solid $[8,28,29]$.

The exact character of the anomalous modes seems slightly less clear, however. Theoretical considerations by Wyart predict that they have a character different from simple plane waves $[28,29]$. In fact, many wave vectors seem to participate in anomalous modes [30]. Furthermore, their appearance is described to be causal for the plateau in the density of states above $\omega^{*}$, while below it plane waves dominate the spectrum. At least for frequencies around $\omega^{*}$ the interplay between anomalous modes and plane waves is not yet clear. Silbert et al. [30] see for jammed packings that waves are not strongly plane wave already in the regime $\omega<\omega^{*}$. The prediction holds for contact networks created through compression (see section 2 ) in which after creation contact forces are relieved through exchange with relaxed springs [31]. This is an important model system for our considerations. Results on relaxed spring networks under temperature are presented in section 5 . 


\subsubsection{The Jamming Phase Diagram}

This section will present a concept that unifies jammed and glassy systems. The obvious unifying element for glasses and jammed materials is the disorder in the structure of their constituents.

Glasses show a liquid-solid phase transition which is induced by lowering the temperature. Yet, jammed systems, like a pile of sand or a package of coffee beans, are in general mesoscopic and athermal: temperature-induced fluctuations are negligible because their interaction potentials are large compared to thermal energies of order $k_{B} T$.

Foams, highly deformable bubbles in a liquid, are athermal too and additionally exhibit a finite elastic response to shear stress up to a maximum where the foam will flow again. The contact interactions of these systems are repulsive.

So far, our considerations have been restricted to athermal jammed systems considering soft sphere packings and their properties. Liu and Nagel in 1998 made an attempt to connect phenomena of athermal jammed systems with observations concerning liquids and glasses [5]. For doing this they loosened two constraints on what is to be considered a jammed system:

\section{No attractive interactions}

2. Individual particles are large so that there is no thermal motion.

A system with attractive forces shows similar behaviour to a system with repulsive forces in a box which exerts external pressure [32]. Jammed systems become rigid with increasing density. On the other hand, supercooled liquids form glasses when the temperature is further decreased below a critical value. In both cases a similar behaviour is observed: a system which is flowing in the beginning responds to a change of an external macroscopic parameter such as temperature or pressure. The system becomes more viscous and, at some point, suddenly hardens. It is then trapped in a small region of phase space and its structure is amorphous. This process is reversible because lowering pressure will lead to unjamming heating will lead to liquefaction.

There seems to be a common denominator for the described amorphous systems: an external control parameter governs the path to rigidity, which is a confined state in phase space. Thus, inverse density, which is the result of external uniform pressure, and temperature are two of the axes in the phase diagram the authors drew. The third axis is external shear stress. As an example one might think of foams that unjam when the applied shear stress exceeds a certain threshold. Liu and Nagel synthesized all these considerations in the phase diagram for jamming, see Figure 1.3. 


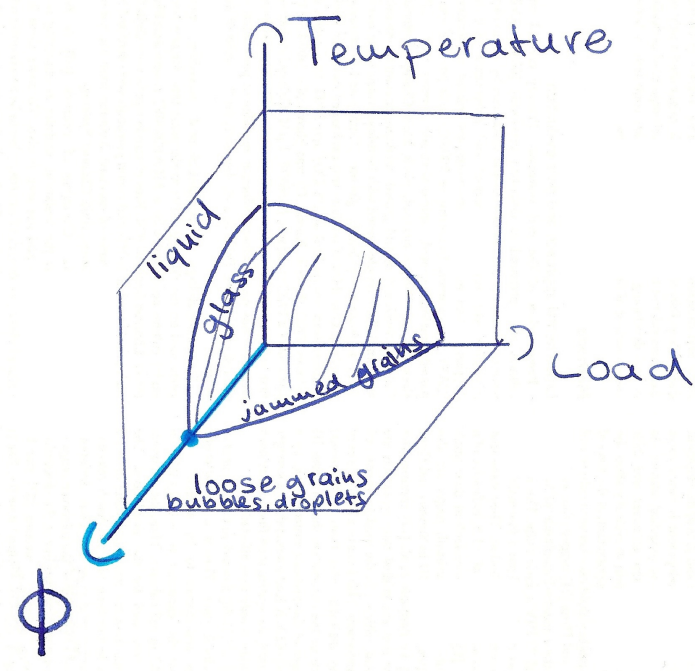

Figure 1.3: Jamming phase diagram for repulsive particles. The jamming axis - marked in light blue - is scaled with inverse density of particles. The other axes are temperature and shear load. The connection between amorphous materials of different nature is hypothesized. Adapted from [5].

The jamming diagram includes a conjecture about how the three parameters inverse density, temperature and stress relate to each other.

The diagram includes the hypothesis that jammed materials begin to flow when a sufficiently high temperature is introduced. The temperature might be considered an effective temperature on a mesoscopic scale. Shaking movements, which are mesoscopic vibrations, that make a jammed systems flow again are an example for such a "temperature". Furthermore, the diagram suggests that an increase in shear stress would decrease the temperature at which glass melts.

All in all the diagram might be called a motivational piece to unify existing findings on amorphous materials. It comes in a handy pictorial form with the intention to spark off new research. In the study presented here we will create athermal jammed packings and probe their dependence on external pressure and temperature. We are mainly interested in the effect of very low, finite temperatures on the packings and their contact networks represented by harmonic springs. This is particularly interesting because temperature will excite the anomalous modes (see section 1.1.5 of the jammed systems). We sketch this approach in Figure 1.4. 


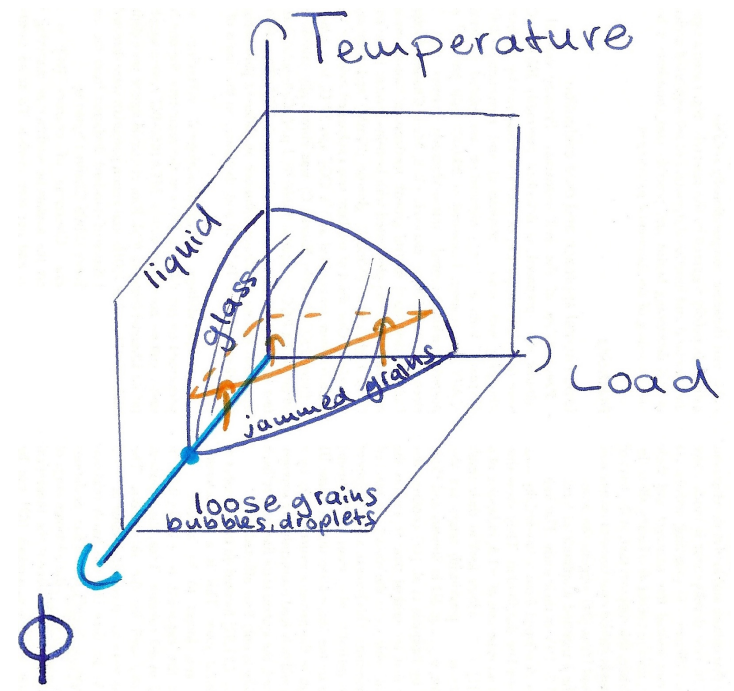

Figure 1.4: Our mission. We consider jammed systems of different coordination (directly connected to the inverse packing fraction quantified by the blue axis) and investigate their response to external tension and finite temperatures. In this sense we move up in the plane as marked by the yellow arrows.

\subsection{Glasses}

The jamming diagram sets out the possible connection between jammed materials and glasses. Yet, it is not purely speculative. The amorphous structure is a strong link between granular material, colloids, foams and glass. The response to thermodynamic and mechanical control parameters gives an additional hunch on how similar their phase transitions are. This section will give more evidence on the connection.

Despite the similarities, atoms of glasses are not spherical and interactions andn be attractive and long-ranged. Hence, the properties of glasses and the jammed soft sphere packings have to be observed closely in order to assess how parallel they are. The focus will be the vibrational modes of glasses and how they relate to the findings of Section 1.1.5.

When a glass is blown in such a way that its cooling is sufficiently quick it moves into a metastable, supercooled state instead of crystallizing. Further decrease of temperature leads to evermore arrested dynamics of the supercooled liquid. Below a specific temperature $T_{G}$ the liquid will have rigid mechanical properties on all timescales of relevant length. This is the glass transition temperature which marks the glass transition. The liquid is now in the glass state. The 


\section{Introduction}

cooling process is called vitrification. The glass transition temperature depends on the material and the rate at which the cooling happens [33]. It is believed that a crystalline groundstate, reachable through a second-order phase transition, exists for glass, but is practically unreachable due to the quenched dynamics. In order to arrive at this underlying crystalline groundstate, the cooling would have to be infinitesimally slow. The practical interpretation of this hypothesis can be debated. As a last remark we want to emphasize that the glass transition is marked by dynamics out of equilibrium in which the history of the system is important [34].

Glasses have a higher density of states for low-frequency modes compared to the Debye-behaviour for the density of states of acoustic modes, which are seen in most crystals. This phenomenon, the boson peak [35], is long-known [36]. At low temperatures this results, for instance, in a higher heat capacity and lower thermal conductivity than what is expected from a crystal [37,38]. Glasses store more heat and transport less of it than crystals. Silica, $\mathrm{SiO}_{2}$, exists in crystalline and amorphous, glassy form. Experiments show that for low temperatures around $25 \mathrm{mK}$, the specific heat capacity of silica glass is more than 1000 times larger than that of crystal [38]. Also, the heat capacity for glasses scales linearly with T instead of $T^{3}$, which is the prediction of Debye's model [39]. It is widely believed that the atomic motions which are associated with the boson peak are indispensable for understanding this particular behaviour.

Strong theoretical and experimental evidence exists that the boson peak can be understood with the concept of soft modes (1.1.5). Brito and Wyart [40] show that in hard sphere liquids rearrangements happen during vitrification along soft modes. This implies that rearrangements leading to structural relaxation happen as extended collective motions instead of localized events [41].

\subsubsection{Silica}

Silica is an important material to probe glasses experimentally. Trachenko et al. [42] use a model which describes silica as $\mathrm{SiO}_{4}$-tetrahedra which are connected through joints. The tetrahedra are rigid compared to the forces of the rather flexible joints. The joints are modelled as loaded harmonic springs with zero equilibrium length and a spring constant tuned to mimic experimental results. This leads to rigid unit modes, which are rotations of tetrahedra without deforming. They are the lowest vibrational energy modes in the system.

We apply Maxwell's criterion for stability to the tetrahedral structure. In total, each tetrahedron has 6 degrees of freedom for translation and rotation. Then, each bridging oxygen at the corner of a tetrahedron imposes 3 constraints. This gives 6 degrees per tetrahedron as every oxygen is shared by two tetrahedra. As 
no degree of freedom is left, we conclude that the whole system is isostatic. When the pressure on such a system is increased, so called five-fold defects occur. This means that the number of five-fold Silicon atoms increases. This way the whole system is departing from isostaticity and the effect on vibrational modes can be studied [43]. It shows that the excess of modes shifts away from low frequencies much like in sphere packings [25].

Silica glass [42] and the corresponding crystal [44] with the same chemical composition show a similar density of states, i.e. with a boson peak. We can draw the conclusion that order cannot be the crucial factor which determines the density of states, for the glass is amorphous and the crystal's structure is ordered. Additionally, because it is very similar, the vibrational spectrum alone cannot account for the peculiar behaviour of glasses compared to crystals. Wyart $[29,45]$ proposes that it is:

1. the coordination instead of positional disorder which matters for the lowfrequency spectra [26] and

2. the exact nature of the modes which is decisive and which is affected by disorder [29].

For crystals anomalous modes come as plane waves. For amorphous solids the anomalous modes are very heterogeneous. Hence there is a difference between crystals and glasses and anomalous modes are the decisive factor in their different thermodynamic behaviour [46]. This view is not undisputed and the exact nature of the boson peak in silica is subject to current research and debate [26, 47].

Chen et al. [48] tested in lab-experiments the validity of the results for the vibrational spectrum of the soft sphere model (see section 1.1.5). They used a bi-disperse mixture of Poly(N-isopropylacrylamide) (NIPA) microgel particles, which swell with decreasing temperature. Consequently, temperature is used to tune the packing fraction of the jammed system. They found that the vibrational properties are in good agreement with those in athermal soft sphere packings: Firstly, they are in the excess of low frequency modes which extends to lower frequencies when approaching the jamming point is approached. Secondly, the same nature of the modes as seen in the soft spheres was recovered: at low frequency, modes are quasilocalized; at intermediate frequencies, modes are highly disordered and extended; at high frequencies they are localized [48].

The exact process of the melting of a sphere packing is not trivial. One of the questions is how a particle can escape its position if it is fully enclosed by other particles. The collective motions of the neighbouring particles which are needed for this to happen are a subject of ongoing research [49]. 


\subsection{Negative Thermal Expansion}

In this section we discuss several aspects of negative thermal expansion. The simplest example is the Gough-Joule effect alread found the 19th century. It sheds some lights on the thermodynamics of negative thermal expansion in one dimension. In two dimensions rotations of rigid units, as introduced in the last chapter, play a decisive role. Three-dimensional models are discussed towards the end of this section.

\subsubsection{Rubber elasticity}

Rubber elasticity is a good example for negative thermal expansion in one dimension [50]. Of course, a real rubber band is not one-dimensional but the effect itself is. A rubber is made of a network of long polymer-chains instead of crystalline ordered atoms. Putting stress on the rubber band, it gets stretched balancing the gravitational force on the weight with its elastic Hookean force. In that process it will emit heat. Considering the Gibbs Free Energy helps to further understand the situation:

$$
T \Delta S=\Delta H-\Delta G,
$$

where $T$ is the absolute temperature. In the case of the stretched rubber band the change in enthalpy $\Delta H$ is negative due to the emission of heat and the change in Gibbs-energy $\Delta G$ is positive because the process absorbs energy. Then, the change in entropy $\Delta S$ can only be negative to match the equation: the entropy of the rubber band is decreasing. Stretching leads to less possible arrangements of the chains in the rubber band. If we now heat the rubber band, it contracts [51]. This is known as the Gough-Joule effect. It happens, because with temperature, the entropic forces are increasing. The less the chains are stretched, the more configurational states are possible. In other words, with decreasing length $l$ of the rubber band its configuration space is increasing. Entropic force favors a larger space of microstates and thus leads to contraction.

Cooling of the rubber band, on the other hand, leads to its expansion. The rubber band exhibits negative thermal expansion along the direction of stretching, i.e. the direction of gravitation. This effect is not unique to rubber bands, though. Also other polymers exhibit contraction on heating, where [52] is an intersting example with a particularly high coefficient of negative thermal expansion. 


\subsubsection{Negative thermal expansion due to network rotations}

Interestingly, some glasses, especially silica, exhibits a negative thermal expansion coefficient. In 1.2 the vibrational spectrum and the character of the vibrations was already discussed. They were attributed to rigid unit modes which are rotations of essentially stiff atomic tetrahedrons, where the terahedrons are connected through a harmonic potential.

Heine et al. [53] showed how isotropic negative thermal expansion can be explained through geometric effects in framework structures: materials which are made up of octahedral or tetrahedral crystal units that are stiff compared to the forces connecting their corners. Examples are many aluminosilicates and the ceramic zirconium tungstate $\mathrm{ZrW}_{2} \mathrm{O}_{8}$. In their calculations the authors [53] compute the overall effect of the rotations in two dimensions of a framework structure to negative thermal expansion. The framework structure is represented as square boxes connected at their corners and referred to as 2D-perovskites.

In the simplest consideration of this model all the squares rotate with the same Einstein frequency $\omega_{\text {rot }}$. $\theta$ is the angle of the rotations. The relative change of the area $A / A(\theta=0)$ in dependence of $\theta$ is in lowest order given by:

$$
\frac{A(\theta)}{A(\theta=0)}=1-\theta^{2}
$$

Equipartition theory tells us how the average potential rotational energy of a unit with inertia $I$ relates to temperature $k_{B} T$ :

$$
\frac{I \omega_{r o t}^{2}\left\langle\theta^{2}\right\rangle}{2}=\frac{k_{B} T}{2}
$$

This gives for the relative change of the area due to thermal fluctuations:

$$
\left\langle\frac{A}{A(\theta, T=0)}\right\rangle=-\frac{k_{B} T}{I \omega^{2}}
$$

Increasing temperature decreases the overall area of the system. The results give some insights on the phenomenon:

1. the effect is geometrical, due to a rotation-induced folding of structures.

2. the relative change in area scales with $1 / \omega^{2}$, hence low-frequency rotations contribute stronger to the temperature-induced contraction.

However, the calculations make some approximations, too: 


\section{Introduction}

1. anharmonic interaction potentials between atoms, which are not considered here, lead to thermal expansion.

2. all modes have been coarsely packed into a single frequency, instead of summing over the vibrational spectrum.

3. the vibrational spectrum itself is temperature-dependent, consequently negative thermal expansion is connected to regimes of certain temperatures.

Heine et al. [53] tackle these shortcomings of the simpler considerations above and succeed to encoparate the geometric effect in Grüneisen theory of thermal expansion. The overall results, though are supported by the more detailed calculations of Heine et al. and we refer to them in case of deeper interest.

Another example of a material that exhibts negative thermal expansion is scandium trifluoride $S c F_{3}$, that consists of $S c F_{6}$ octahedra, which share a corner [54]. The scandium atoms sit at the centre. It exhibits negative thermal expansion over a large range of temperature from $10 \mathrm{~K}$ to about $1100 \mathrm{~K}$ [55]. In contrast to Silica and the rigid unit modes model in $S_{c} F_{3}$ during vibrations, the connection $S c-F-S c$ between two scandium atoms is stretched. The potential energy of the interaction is quartic, i.e. $x^{4}$ with $x$ being the transverse displacement of the fluoride atom. Li et al. [56] find that the quartic potential of interaction between the octahedra accounts for significant part of the negative thermal expansion behaviour.

Negative thermal expansion is a field of active research where especially exact mechanisms behind the phenomenon and the search for materials with the NTEproperty are of interest [57-59].

\subsubsection{Soft mode analysis of jammed spring networks}

The main subject of this research are spring networks obtained from jammed packings under temperature $[11,12,60]$. We want to complement the computer simulations with derivations made from analyzing the anomalous modes of spring networks [61]. The derivations sketched here are based on the analysis of the soft mode spectrum of jammed packings [29].

We present the main steps of the derivation. For this we need to consider affine contributions of the athermal network and non-affine contributions derived from the energy required to excite a soft mode.

The network has a defined distance to isostaticity $\Delta z$ and is subject to a tension $\tau$ (which we specify in units of the spring constant $k_{s p}$, see Equation 3.1).

The affine part $\epsilon_{a}$ of the extension $\Delta A / A=\epsilon_{a}+\epsilon_{n a}$ is direcly proportional to the 

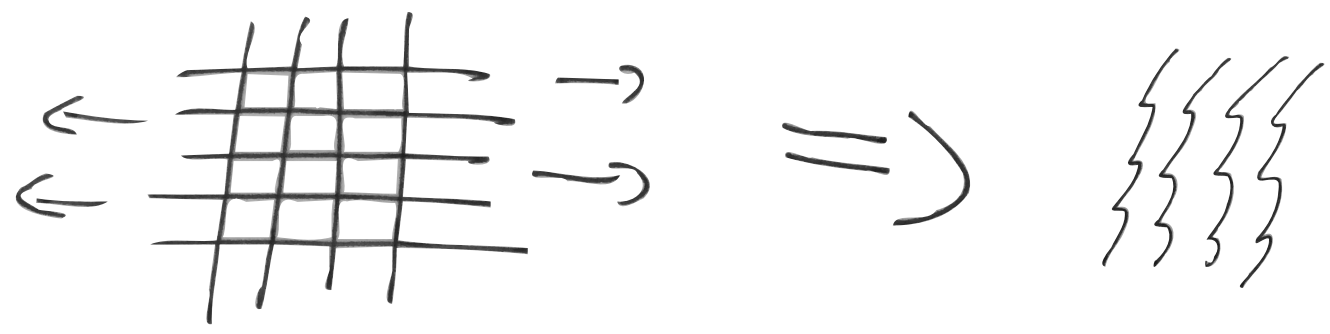

Figure 1.5: Temperature excites soft vibrational modes of the system which lead to contraction of the system. We call this the squeezebox-effect. Here we show how the chains in one direction of a square lattice get contracted. In this state an external tension will lead to stretching the zig-zag folding of the line instead of affecting individual bonds.

coordination number $z$ of the system and the external stress:

$$
\epsilon_{a}=\frac{\tau}{4 z}
$$

From [29] we have a predicition for the energy to excite a soft mode. It turns out, that the excitation of soft modes leads to a large negative contribution to the non-affine part of extension [61], as long as tension is small, $\tau \Delta z^{-2} \ll 1$. This can be most simply understood intuitivley when considering a fourfold network Figure 1.5.

Summing up the affine and non-affine contributions gives a prediction for the extension with respect to $T, \Delta z$ and small tension $\tau$ :

$$
\frac{\Delta A}{A}=\epsilon_{a}-\epsilon_{n a}=\frac{\tau}{4 z}-\frac{T}{4 \Delta z}\left(1+\tau \Delta z^{-2}\right)^{-1} .
$$

The bulk modulus is obtained from the first derivative of the relative extension with respect to tension $\tau$ :

$$
\frac{1}{B}=\frac{1}{B_{0}}+\frac{T}{4 \Delta z^{3}}\left(1+\tau \Delta z^{-2}\right)^{-2}
$$

When tension dominates, $\tau \Delta z^{-2} \gg 1$, we get for the extension [61]: 
1 Introduction

$$
\frac{\Delta A}{A} \approx \frac{\tau}{4 z}-\frac{T}{8 \sqrt{\tau}} .
$$

By differentiation the bulk modulus becomes:

$$
\frac{1}{B} \approx \frac{1}{4 z}+\frac{T}{16 \tau^{3 / 2}} .
$$

The athermal shear modulus $G$ is proportional to $\Delta z$ [62] for vanishing tension:

$$
G \sim \Delta z,
$$

and

$$
G \sim \sqrt{\tau},
$$

for dominating tension. At fixed volume thermally activated soft modes will lead to an internal pressure. To obtain it we set the extension to zero in (1.24), $\epsilon=\frac{\Delta A}{A}=0$ :

$$
\tau \sim T^{2 / 3}
$$

In terms of the shear modulus we thus get for the scaling dominating thermal tension, $T>>\Delta z^{3}$, in the canonical ensemble:

$$
G \sim T^{1 / 3}
$$

If we find the same scalings in the simulations like presented, this is strong evidence for the mode spectrum being correct.

\subsection{Structure of this work}

This work is structured as follows. 
In the next chapter we introduce the athermal protocol which we used to obtain jammed packings and their corresponding spring networks.

Furthermore, we introduce the protocol to obtain pruned networks.

In Chapter 3 we describe the Monte Carlo Metropolis scheme used to simulate jammed spring networks and packings at finite temperatures.

In Chapter 5 we present the results on jammed spring networks at finite temperature and connect the finding to theoretical predictions.

In Chapter 6 we show how the structure of a network influences elastic properties.

In Chapter 8 we consider thermal jammed packings and set the findings into context with the results from spring networks.

We finalize with a discussion of the main results and give an outlook on future research paths. 



\section{Generating jammed sphere packings}

In this chapter we will describe the exact protocol utilized to generate sphere packings. To execute the jamming transition a two-dimensional volume is filled up with soft spheres which interact through harmonic repulsive forces without friction as introduced in chapter 1.1.1. The algorithm is in large parts parallel to the standard introduced by O'Hern et al. [13].

We generate such packings according to the following protocol:

1. Place $N$ particles randomly in a two-dimensional, quadratic box of edge length $L . \mathrm{N} / 2$ particles have radius $r_{1}=0.5$. The other $\mathrm{N} / 2$ have radius $r_{2}=0.7$. $L$ is chosen such that the packing fraction $\phi=\pi\left(N_{1} r_{1}^{2}+\right.$ $\left.N_{2} r_{2}^{2}\right) / L^{2} \approx 0.7$.

2. Minimise the energy of the system with the lammps [63] software package, making use of its implementation of the Polak-Ribière version of the conjugate gradient algorithm in the minimize function.

3. Measure the pressure - defined in Eq. (4.14) - and the coordination number $z$ of the system. As explained in Chapter 2.1.

4. Increase the packing fraction by slightly inflating the radii of the spheres in the packing by a common factor $\alpha_{\text {compress }}$.

5. Repeat steps 2 - 4 until coordination number $z=6.0$.

6. Decompress the packing fraction by deflating the radii of the spheres in the packing slightly by the same factor $\alpha_{\text {deflate }}$. The factor $\alpha_{\text {deflate }}$ should be chosen small compared to the packing fraction so that the sampling is dense in $\phi$. Furthermore, rearrangements due to a large release of pressure are less likely this way.

7. Repeat steps 2,3 and 6 and record the resulting packings at each level of adjusted packing fraction until coordination number drops to zero.

The spheres are bi-disperse to avoid crystallization. The described protocol generates a number of sphere packings which should be in the same arm of energy minimisation for different $z$. Rearrangements during deflation are minor and rep- 

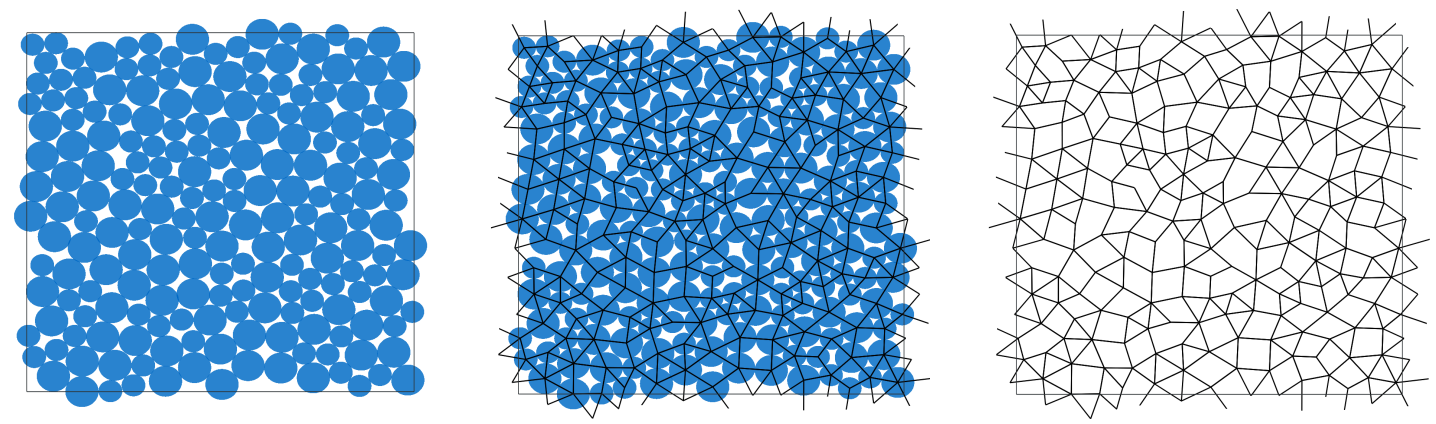

Figure 2.1: From jammed packing to contact network at coordination $z=4.60$ with system size $N=200$. The left side shows a jammed packing obtained by the protocol described in the text. In the middle touching spheres define a contact between spheres. The spring network is generated by connecting the centres of the interacting spheres. We keep only the network and leave aside the extent of the spheres.

resent the simplest movement to realize a lower pressure. The idea is to avoid redistribution of the spheres to a completely different jammed state.

Figure 2.1 shows a jammed packing obtained by the protocol. Touching spheres define a contact between spheres. Such contact networks will be used for major parts of this work. Each connection in the contact network describing a harmonic spring.

Figure 2.2 shows four examples of the contact network's structure of jammed packings at different average coordinations.

By visual inspection, the networks are isotropic. For large packing fractions $\phi$, (Figure $2.2 \mathrm{~d}$ ) the contact network is very fine and most vertices are five- or six-fold connected. With decreasing packing fraction the connectivity lowers and holes start appearing in the contact network. Holes are a sign of spheres in the packing which are not connected, i.e. rattlers.

The total fraction of rattlers depends on the packing fraction of the jammed packing and amounts to approximately $5 \%$ of the spheres in the packing close to the isostatic point, see Figure 2.3.

Figure 2.4 shows the development of coordination number, energy and pressure as a function of the packing fraction. When inflating the spheres there are configurations of packing fractions below the isostatic point which are stable towards 

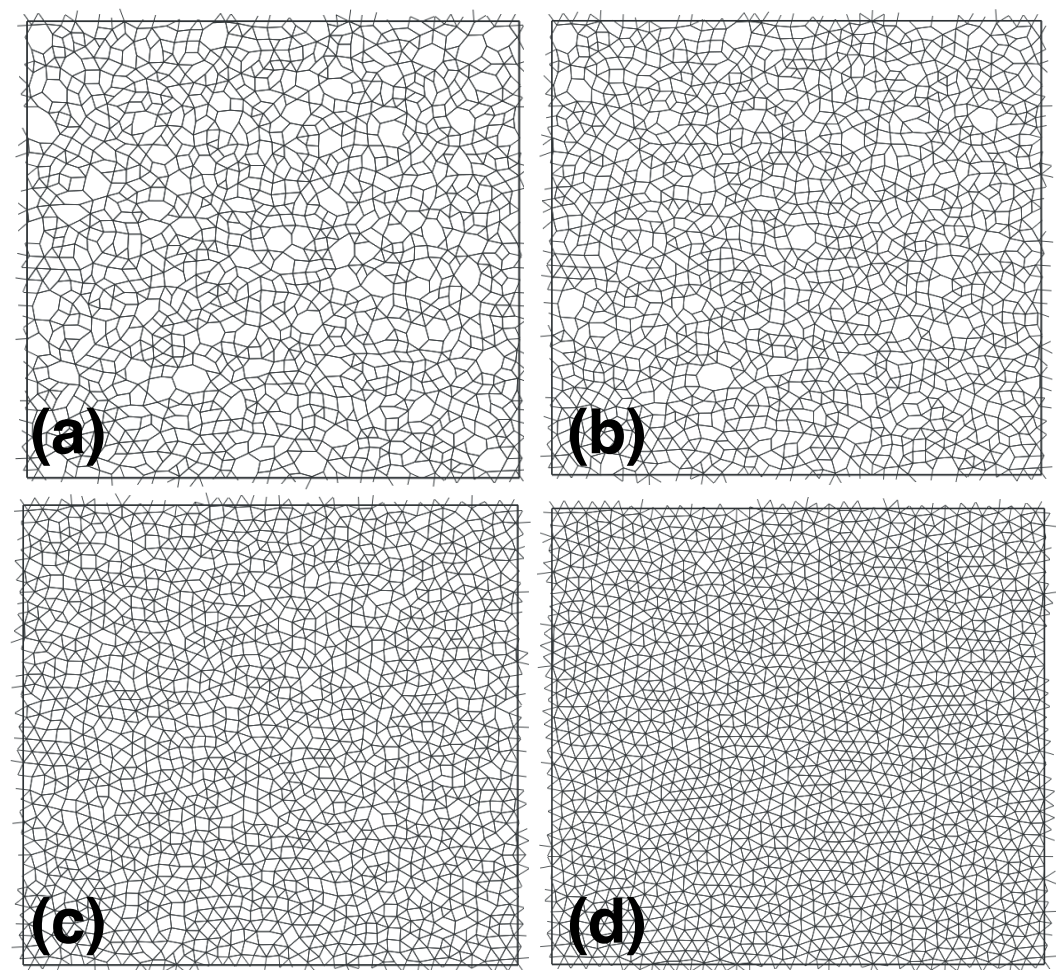

Figure 2.2: Four examples of the contact network's structure of jammed packings obtained from the described protocol. System size $N=1600$. a) Coordination $z=4.0013$ b) $z=4.3921$ c) $z=5.1137$ d) $z=5.9900$

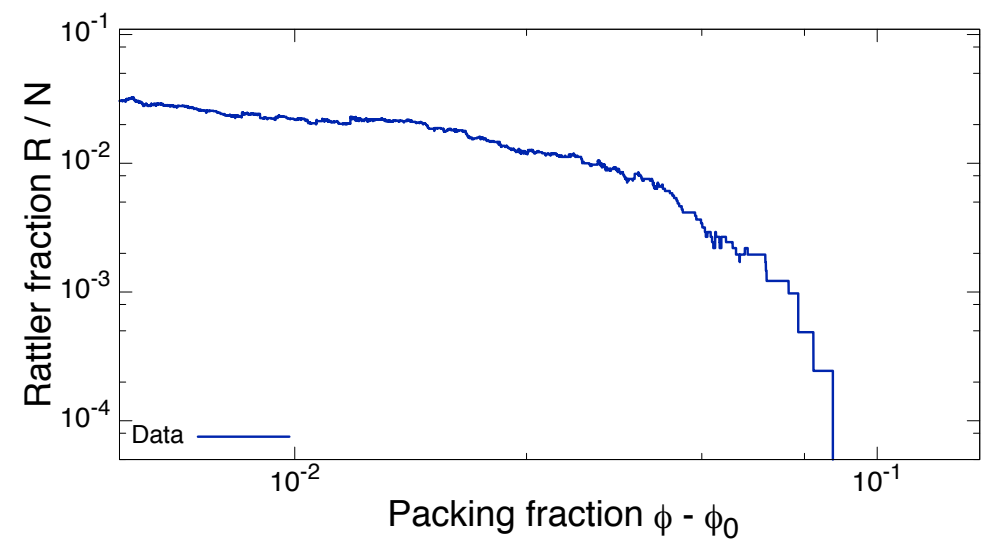

Figure 2.3: Rattler fraction for a packing of size $N=4096$. Close to isostaticity $\phi \rightarrow 0$ the fraction of rattlers is around $5 \%$ and then decays quickly. 
energy minimisation. However, any thermal fluctuation would destroy those configurations because they are highly under-coordinated. This can be seen as well in the relatively small to vanishing energy and pressure which is stored in these packings. Using the packings obtained out of decompression however we get packings which are stable to (in the sense of this work) small thermal pertubations.
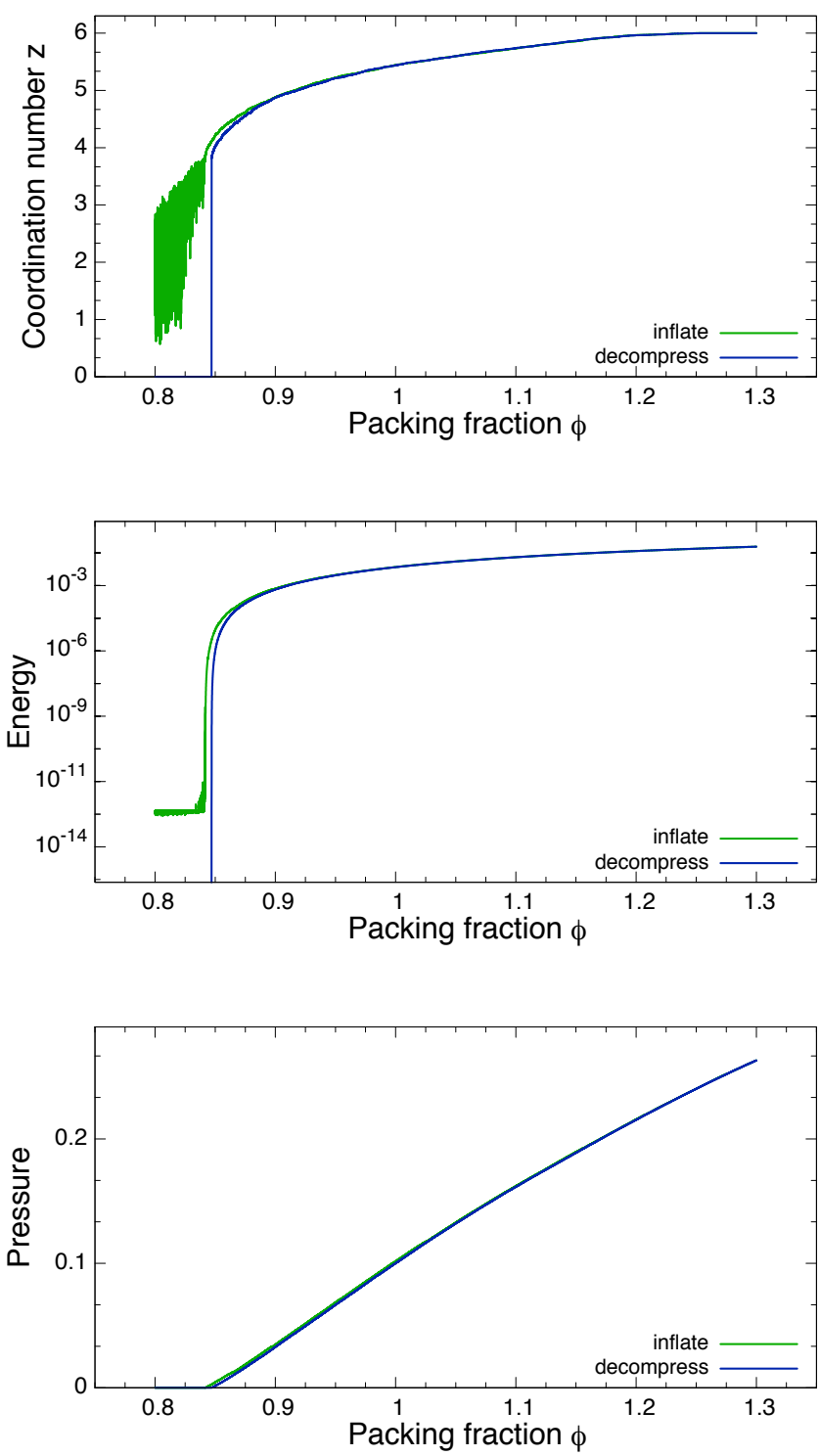

Figure 2.4: Coordination number, reduced energy in units of $k_{s p} R_{i j}^{2}$ and pressure as a function of the packing fraction. System size $N=4000$. Details in main text. 


\subsection{Measuring coordination}

In practice measuring coordination is slightly more involved than presented in Equation 1.5. For instance it needs to be defined below which number of contacts a sphere is identified as a rattler.

A link between vertices $i$ and $j$ is established when the spheres overlap:

$$
Z_{i j}=\left\{\begin{array}{lr}
1, & \text { if } d_{i j}<R_{i}+R_{j} \\
0, & \text { otherwise }
\end{array}\right.
$$

This gives for the number of links of $i$ :

$$
Z_{i}= \begin{cases}\sum_{j} Z_{i j}, & \text { if } \sum_{j} Z_{i j}>1 \\ 0, & \text { otherwise. }\end{cases}
$$

Total number of non-rattler links in the system:

$$
Z=\sum_{i} Z_{i}
$$

The rattler function is defined as:

$$
R(i)=\left\{\begin{array}{lc}
1, & \text { if } Z_{i}=0 \\
0, & \text { otherwise }
\end{array}\right.
$$

We use $R(i)$ to get the total number of rattlers:

$$
R=\sum_{i} R(i)
$$

The average coordination is then:

$$
z=\frac{2 Z}{N-R}
$$




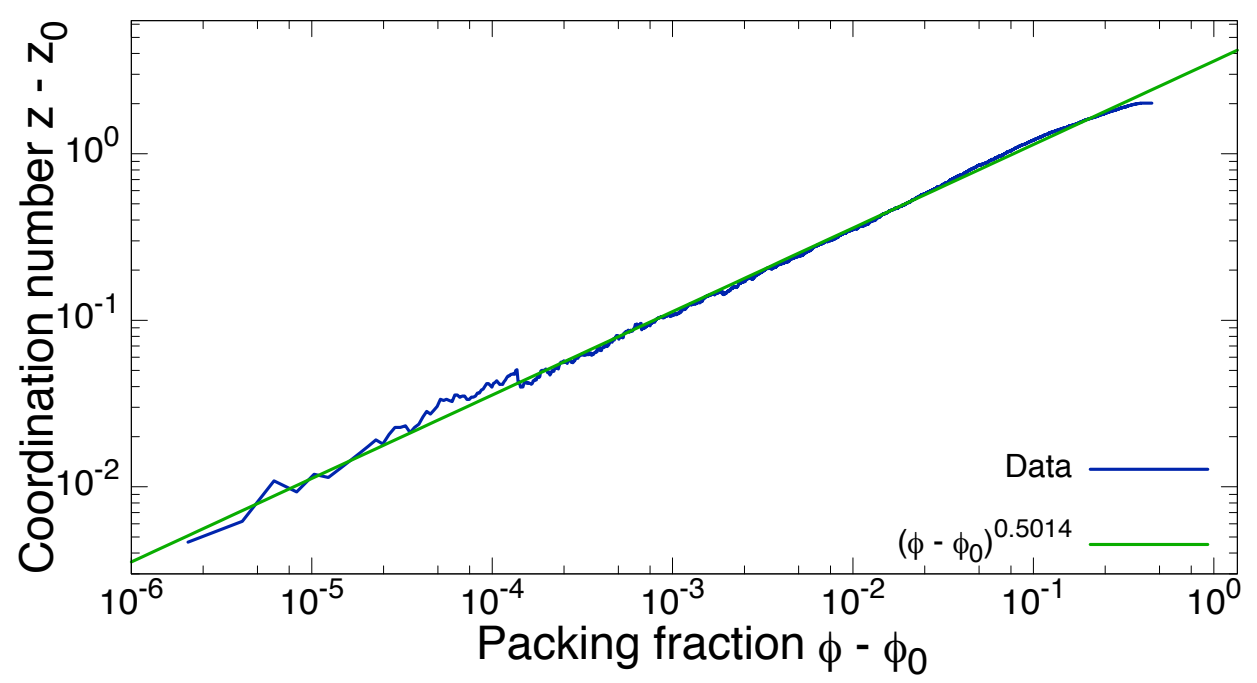

Figure 2.5: Scaling of the coordination number's distance to isostaticity with the packing fraction minus its isostatic values. The fit for the exponent delivers $0.5014 \pm 0.0003$, which restores the square root scaling of $\Delta z[17]$.

\subsection{Identification of the critical coordination number}

In the introduction we discussed the square-root scaling of the coordination number for jammed packings (1.13).

To verify this for the packings we generate we need to identify the critical packing fraction $\phi_{0}$ and critical coordination number $z_{0}$ first. In a finite system $z_{0}$, for instance, is slightly smaller than 4.0 [16]. Also, the finite minimisation steps for one packing fraction and the finite spacing when lowering $\phi$ leave residual configurations which are actually unstable.

We identify $\phi_{0}$ and $z_{0}$ by probing pairs $(\phi, z)$ which are close to the drop in pressure seen in Figure 2.4 as fit parameters. The best fit for $\left(\phi-\phi_{0}\right)^{1 / 2}=z-z_{0}$ will give us $\phi_{0}$ and $z_{0}$. Figure 2.5 shows the best fit we obtained for the packings obtained from the decompression. 

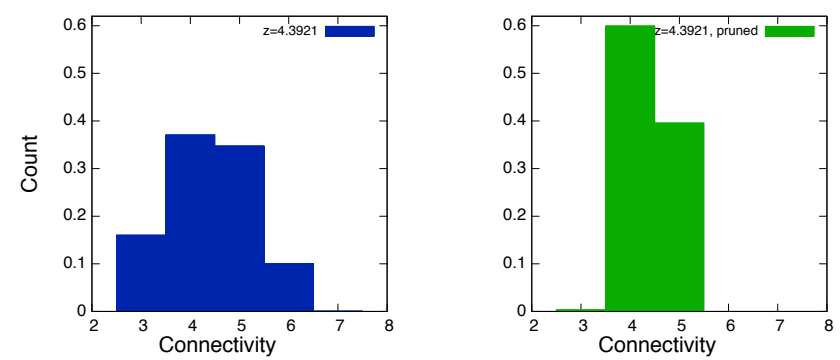

Figure 2.6: Left: Distribution of links for a network directly obtained from a jammed packing.

Right: Distribution of links for a pruned network out of the pruning protocol starting from a packing at $z=5.990$.

\subsection{Pruning protocol}

To complement the protocol that infers spring networks directly from jammed packings we introduce another protocol to initial networks. This is to investigate the effect of structure on thermalized spring networks. In this way we can probe if the coordination number alone is sufficient to describe a spring network's behaviour with temperature or if the way links are arranged is important, too. The study is found in chapter 6 .

The algorithm to generate lower coordination numbers out of networks with a given average $z$ was constructed in the hope of maintaining the main physical properties of the system and to avoid percolation. Such an algorithm is often referred to as a pruning protocol. In the community these networks are often referred to as random networks $[2,64,65]$.

We use the following:

1. Take a given contact network with a certain average $z$.

2. Identify the set of vertices with the highest $z$.

3. Choose a vertex $i$ out of that set randomly.

4. Find the vertices connected to $i$ with the highest coordination.

5. Choose one vertex $j$ out of these and remove the spring connecting $i$ and $j$.

6. Continue until desired average $z$ is reached.

As a result we get contact networks as illustrated in Figure 2.7. The average 
2 Generating jammed sphere packings
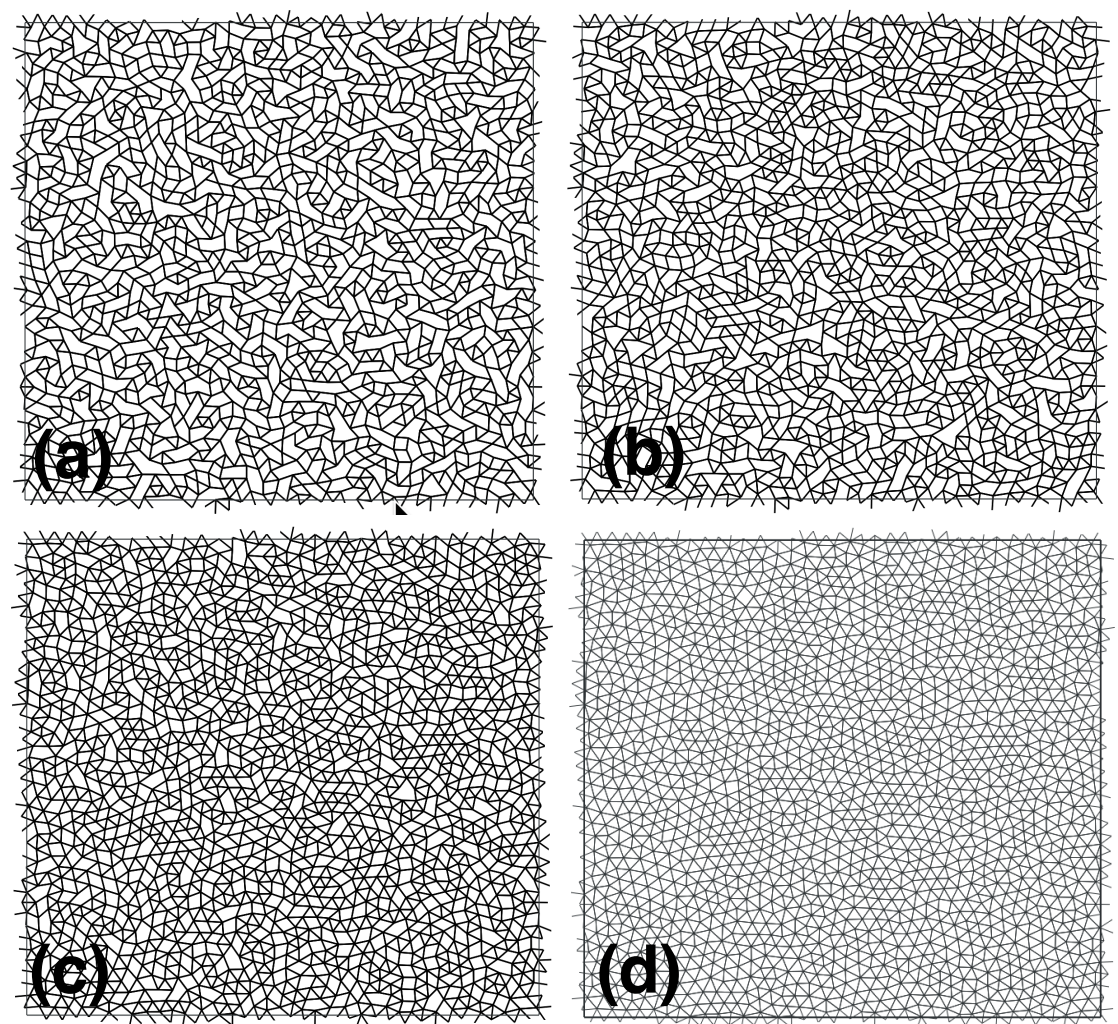

Figure 2.7: Four examples of the contact network's structure of jammed packings obtained from pruning. System size $N=1600$. a) Coordination $z=4.0013$ b) $z=4.3921$ c) $z=5.1137 \mathbf{d}$ ) $z=5.9900$, initial jammed packing configuration obtained from energy minimisation.

coordination numbers are the same as in Figure 2.2. Yet the distribution is different, see Figure 2.6. It is not straight-forward to design meaningful pruning so that the link distribution is maintained. 


\section{Model}

We want to probe the effect of temperature on jammed systems. To do so we need a suitable model system. We focus on thermodynamic properties as we want to determine the contraction in volume of a fixed-pressure system, as well as the bulk and the shear modulus. Of particular interest is the behaviour of spring networks derived from jammed packings as well as jammed packings themselves.

The system is created through athermal energy minimisation. Let's assume that we have a packing created through the energy minimisation protocol described in chapter 2. Spheres form a contact network in which overlapping spheres are replaced with springs connecting the centres of the spheres, while rattlers are excluded. That is how we obatin spring networks. Their structure is fixed through the static connections defining a network of springs. In contrast to thermalized packings no rearrangments or breaking contacts are possible.

The connectivity of the packing yields the first parameter of the system, the dimensionless average coordinaton number $z$.

The spring networks or packings will be in two-dimensional square volume. The boundaries of the volume are determined by the two vectors $U$ and $V$, see Figure 3.1 for their definition. In order to be able to measure reaction to shear we allow the vectors $U$ and $V$ enclosing the area to change [66]. This situation is illustrated in Figure 3.1.

In the case of fixed-volume simulations we can keep the area of the system and only change $U$ and $V$ so that the system gets sheared. In order to simplify the situation, $U$ is always rotated so that it aligns with the horizontal axis.

In the case of fixed-pressure simulations we also allow the vectors to change in length which allows us to measure thermal expansion. Furthermore, we can set a pressure in the system through the external tension $\tau^{*}$. Then we can immediateley define the reduced external tension $\tau$ without dimensions by comparing it to the spring constant $k_{s p}$ as follows:

$$
\tau=\frac{\tau^{*}}{k_{s p}}
$$



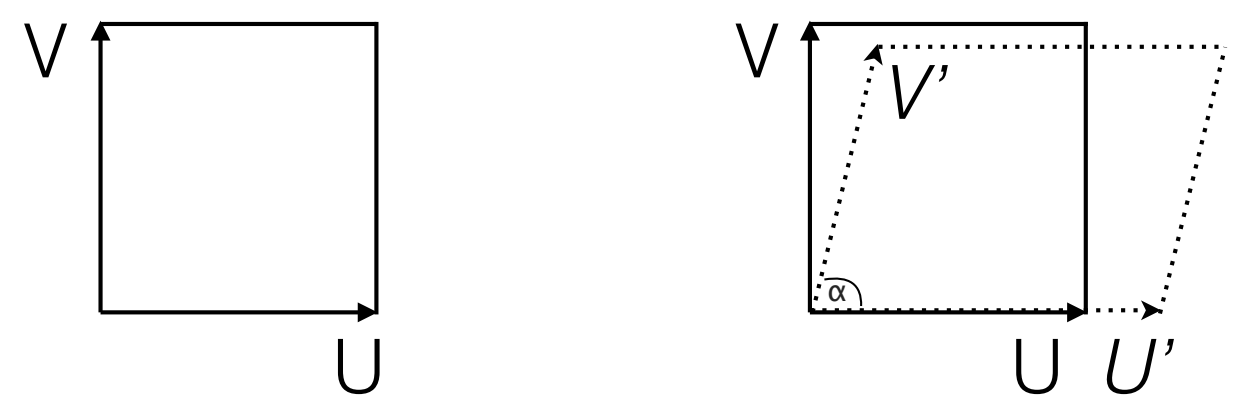

Figure 3.1: Vectors $U$ and $V$ defining the area of our system. In general, only initially the system is a square. By changing $U$ and $V$ to $U^{\prime}$ and $V^{\prime}$ we get a parallelogram with a change in area and angle $\alpha$ defining the shear of the system.

A positive $\tau$ draws the volume apart, resulting in a negative pressure. A negative $\tau$ pushes it together, resulting in a positive internal pressure within the system, see Figure 3.2.

The energy of the spring network at fixed volume is:

$$
E_{n e t}=\frac{k_{s p}}{2} \sum_{<i j>}\left(d_{i j}-R_{i j}\right)^{2}
$$

where the sum runs over all springs between vertices $i$ and $j$. The distance between two vertices is $d_{i j}, R_{i j}$ the rest length of the spring and $A$ the area of the system. The spring constant $k_{s p}$ is commonly set to 1.0 in this study.

In the case of packings:

$$
\begin{gathered}
E_{\text {pack }}=\frac{k_{s p}}{2} \sum_{<i j>} e_{i j} \\
e_{i j}= \begin{cases}\left(d_{i j}-R_{i j}\right)^{2}, & \text { if } d_{i j}<R_{i j} \\
0, & \text { otherwise. }\end{cases}
\end{gathered}
$$

In the isothermal-isobaric ensemble with fluctuating area, the enthalphy reads: 

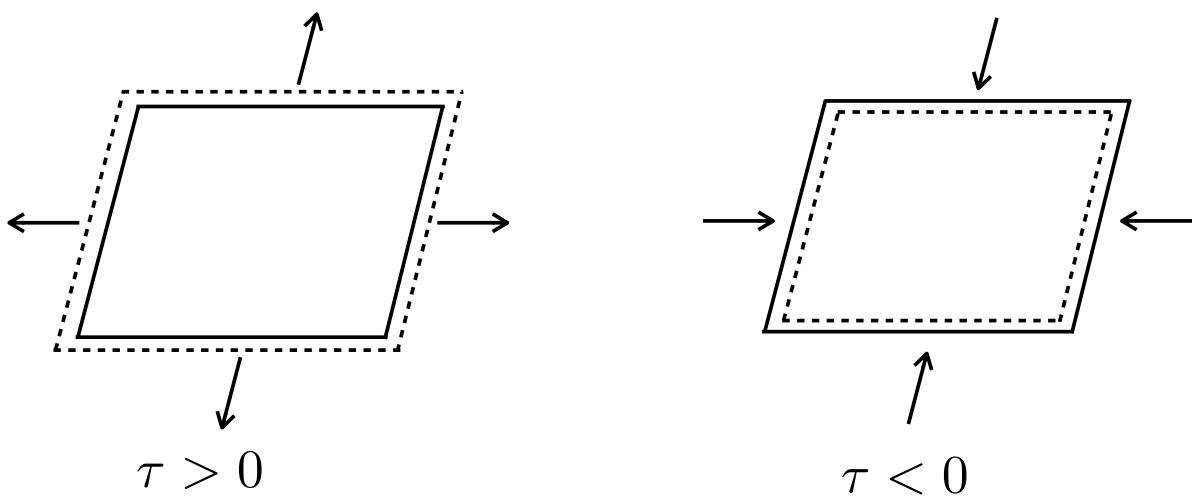

Figure 3.2: The system may be put under positive or negative external tension. If the system collapses with temperature positive tension will support it in staying stable. A negative tension will result in reaching the system's collapse at lower temperatures.

$$
H=E_{n e t}-\tau^{\prime} A
$$

Mainly, we are interested in thermal spring networks and jammed packings at temperature $T^{*}$. The dimensionless temperature $T$ is defined as the ratio of thermal energy over the average energy in the harmonic potentials [66]:

$$
T=\frac{k_{B} T^{*}}{k_{s p}{\hat{R_{i j}}}^{2}}=\frac{1}{\beta k_{s p}{\hat{R_{i j}}}^{2}}
$$

where $k_{B}$ is the Boltzmann factor, which is the probability to find a particle at a certain energy state in thermodynamic equilibrium and $\beta$ is the inverse temperature times $k_{B}$ and $\hat{R_{i j}}$ the average rest length in the system.

This model system allows us to study the effect of thermal excitation on spring networks and jammed packings. The defining dimensionless parameters are the coordination number $z$ and its distance to the isostatic point $\Delta z=z-z_{0}$ respectively, the external tension $\tau$ and the dimensionless temperature $T$.

In order to study equilibrium properties of the described model system we employ a version of the Metropolis Monte Carlo algorithm. Temperatures will be rather low and the situation similar to the encased phase space of a typical glassy system. The details are found in the following Chapter 4 . 



\section{Methods}

The creation of networks or packings as described in 2 introduced how the initial systems are created. We use contact networks formed by the particles in the packings to create spring networks in order to study the effect of temperature on these in the next step.

The algorithms behind the computer simulations to investigate the effect of temperature will be explained in the following. This encompasses a description of the Monte Carlo Metropolis method as well as performance optimisations developed in the course of the project.

\subsection{A small remark on the role of simulations}

Simulations are necessary because we are dealing with a complex model system, which is all but impossible to be fully treated analytically. In order to obtain results for many relevant aspects of the proposed model, computer simulation is the appropriate tool. Nevertheless - as explained in the previous chapter - we have a prediction of how our system behaves within certain parameters. The simulations validate the theoretical predictions and the underlying assumptions in silico. In modern physics computer simulations represent an increasingly important tool and provide new scientific insight - indepedently from theory and classical experiments. It is hard to imagine today how high-dimensional models might be tested and prepared for experimental verification in other ways. However, simulations interplay well with theory and experiments as suggested in Fig. 4.1, in the best case bridging both fields by validating theory and inspiring new experiments.

Among the many advantages of computer experiments is the direct accessibility to all - even microscopic - quantities of the system at hand. The quantities have to be derivable from the used model, of course. In real experiments it can be difficult to access certain observables, like the exact position of each constituent over time. 


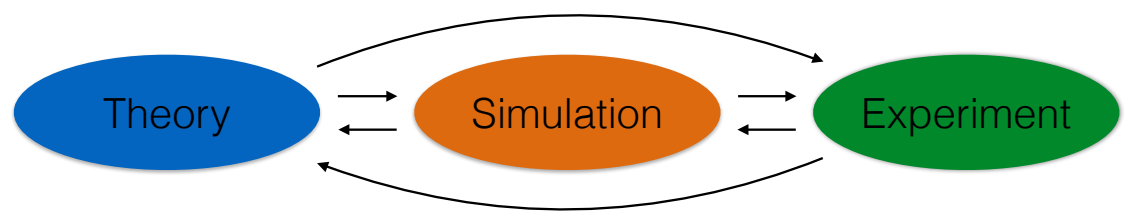

Figure 4.1: Role of computer simulations in modern physics. In the best case simulations validate theory and inspire new experiments. In recent decades computer simulations have squeezed in between theory and experiment becoming an entity of their own.

\subsection{Monte Carlo}

In order to obtain values for the observables in our system - such as the pressure or mechanic moduli - we have to simulate the model. The model was described in chapter 3. As we are only interested in average values of our observables we can make use of Monte Carlo methods in order to sample our system.

Described with a wide lense, Monte Carlo algorithms utilise random trials to sample the state space of the system at hand. Over time this leads to a probability distribution of the system's state space. This distribution can then be used to compute values for the observables we are interested in. The method was introduced in the late 1940s by Stanislaw Ulam [67] within the Manhattan Project. Yet, Monte Carlo algorithms can be generalized to a broad range of applications, such as finance [68], which go beyond physics.

\subsubsection{Theoretical foundations}

The essential idea behind Monte Carlo simulations consists in making the model system at hand propagate through state space so that the statistical properties of each state are represented. This means that, for each point in time, the occurrence probability for a state $\mu$ is equal to its weight in state space at that point in time [69]. The weight is connected to the energy $E$ of the state through the Boltzmann distribution $w(E) \propto \exp \left(-E / k_{B} T\right)$, where $k_{B}$ is the Boltzmann factor.

The trick is to find simulation dynamics which let the system propagate on a path fulfilling this. Letting the system run for sufficiently long simulation times under such dynamics ensures that each state is sampled with sufficient accuracy. As a result, the observables which are derived from the state space statistics will mirror the actual system behavior. 
For large systems - or more precisely systems with a large state space - such as the ones we are dealing with in this work [70], it will be impossible to sample all possible states. Thus, one should make good use of simulation time and only choose the states which actually make a large contribution to the partition function of our system.

But how do we identify these states? Only a small subset of states, i.e. those with a high probability of occurring, contribute significantly to the average. Likewise the real system does not cover all possible states (rather a very small subset) during observation at the laboratory for finite times. The states with a high probability of occurring are those which are associated with low energy compared to the rest of the spectrum. Thus, instead of picking states randomly, we choose them so that low energy states are picked more frequently than those with high energy:

"Instead of choosing configurations randomly, then weighting them with $\exp \left(-E / k_{B} T\right)$, we choose configurations with a probability $\exp \left(-E / k_{B} T\right)$ and weight them evenly."

Metropolis et al. (1953) [71]

Because of their large contribution to the system's behaviour they should be sampled densely. This approach is called importance sampling.

As argued above, it is important to sample the right set of states. Moreover, it is important to see how the system moves through state space: generating loose states randomly is not a good idea, as in this way many states with a low probability of occurrence will be generated. This results in a lot of wasted computation time.

In Monte Carlo simulations an algorithm generates a series of successional states with each state $\nu$ generated out of a given state $\mu$. This strategy defines a Markov process creating the states of our system. As we will see in section 4.3.8, defining an adequate mechanism to create new states is crucial for Monte Carlo simulations.

Our Markov process needs to satisfy two additional properties in order to make sure that the thermal fluctuations of the system are sampled properly:

First, the process needs to be ergodic. This means that for a given state $\mu$ it is possible for our process to reach any state $\nu$ of the system through a chain of reachable states at finite times. Hence, for getting average properties of the system we can use snapshots at certain time intervals instead of following the exact dynamics of the system. The time average can be replaced by the ensemble average. 


\section{Methods}

Second, the process has to fulfill detailed balance:

$$
p_{\mu} P(\mu \rightarrow \nu)=p_{\mu} P(\nu \rightarrow \mu)
$$

where $P(\mu \rightarrow \nu)$ is the transition probability from state $\mu$ to state $\nu$. As a condition the process needs to run long enough, so that it is equilibrated, i.e. has overcome an initial bias in its configuration. Then detailed balance ensures that we can demand the Markov chain to create states $\mu$ with the desired Boltzmann probability distribution $\propto \mathrm{e}^{-\beta E_{\mu}}$ (See [69], Chapter 2.2.3 for details), where $\beta$ denotes the dimensionless inverse temperature.

If both conditions are fulfilled, the simulation moves through state space as the real system does. However, it has to be emphasized again that this does not mean that the trajectory through states of our simulation itself is necessarily real or physical. The simulation dynamics are primarily those of the Monte Carlo algorithm, not the real dynamics. Yet, this should be unproblematic as we have already declared that only average values are of interest and not the dynamics.

\subsubsection{Monte Carlo Metropolis}

The transition probability from one state $\mu$ to another state $\nu$ is the product of the probability $g(\mu \rightarrow \nu)$ that our program chooses $\nu$ when it is in $\mu$ and the acceptance probability $A(\mu \rightarrow \nu)$, which is our freely tunable parameter and determines if our program accepts a newly created step $\nu$ or not:

$$
P(\mu \rightarrow \nu)=g(\mu \rightarrow \nu) A(\mu \rightarrow \nu)
$$

The condition for detailed balance, Equation (4.1), leaves freedom in the choice of the transition probabilities as long as the following ratio holds:

$$
\frac{P(\mu \rightarrow \nu)}{P(\nu \rightarrow \mu)}=\frac{g(\mu \rightarrow \nu) A(\mu \rightarrow \nu)}{g(\nu \rightarrow \mu) A(\nu \rightarrow \mu)}
$$

Additionally, we picked the Boltzmann distribution for the probabilities, thus:

$$
\frac{g(\mu \rightarrow \nu) A(\mu \rightarrow \nu)}{g(\nu \rightarrow \mu) A(\nu \rightarrow \mu)}=e^{-\beta\left(E_{\nu}-E_{\mu}\right)}
$$

In the famous Metropolis version of the Monte Carlo algorithm [71] the selection probabilities $g(\mu \rightarrow \nu)$ are equal for all possible states and otherwise zero. Moving from $\mu$ to $\nu$ or vice versa does not matter either. Furthermore, without loss of 
generality, we take the energy $E_{\mu}$ of state $\mu$ to be smaller than $E_{\nu}$. We can now set $A(\nu \rightarrow \mu)$ to be 1 . Thus, we do not waste any computation time if we find a lower energy state because we will always accept it. We still have to satisfy (4.4) in order to end up then with the following acceptance probability:

$$
A(\mu \rightarrow \nu)= \begin{cases}1, & \text { if } E_{\nu}<E_{\mu} \\ e^{-\beta\left(E_{\nu}-E_{\mu}\right)}, & \text { otherwise. }\end{cases}
$$

Essentially, we use the Monte Carlo Metropolis algorithm with an acceptance probability as in (4.5).

\subsection{Implementation}

In the following we will describe the specifics of the algorithm in use and its implementation.

\subsubsection{Boundary conditions}

The volume of the simulation has periodic boundaries, which constitutes a topological torus: when a vertex or sphere moves over the border at one side it appears at the opposite side of the simulation box. This procedure approximates an infinitely large system. In the case of the vertices, where each vertex is connected through a spring, the measurement of the spring length can be slightly more cumbersome.

Yet, the vertices move and the shortest distance between two vertices may change its character: at the beginning of the simulation it is shortest via one of the borders of the box. Later, when the vertices move a lot it might be shortest when simply taking the cartesian distance inside the box. Hence, in theory we need to record how the initial distance was measured. Only then can we later measure the distance correctly when computing the energy contribution of the spring to the system's Hamiltonian.

In practice, though, the movements of the vertices are small compared to the size of the box as we are interested in the low-temperature behaviour of the system. Consequently, it is sufficient to compute the shortest distance with respect to the periodic boundaries between two vertices.

The simulation box is generally not a square, but a parallelogram in the simulations conducted, see Figure 3.1. The fluctuations of the opening angle of the simulation box allow to measure the shear modulus of the system, see section 4.3.9. 


\section{Methods}

In order to conveniently handle the coordinate transformations connected to the boundary conditions, a coordinate system of unity edge length is used. For calculations of the energy and other physical quantities these coordinates are folded back to physical coordinates.

\subsubsection{NVT simulation}

One part of the simulations is conducted in the canonical ensemble, where the number $N$ of entities being vertices or spheres as well as the volume $V$ and the temperature $T$ are fixed. Here, it will usually be denoted by NVT. In each simulation step one of the $N$ entities in the system is chosen randomly. Next, a Monte Carlo trial move is performed by displacing the entity to a randomly chosen position inside a square box of a certain edge length. If the energy of the system is smaller after the trial move the algorithm accepts the move. If not, the algorithm accepts the move with the Boltzmann probability according to (4.5).

The edge length of the box in which we choose a new step is called stepsize. The stepsize is a critical parameter for the performance of the computer simulation. It might be fixed a priori or changed in the course of the simulation. In subsection 4.3.8 the algorithm to choose it automatically is explained.

\subsubsection{NpT simulation}

In NpT simulations the number $N$ of entities being vertices or spheres is fixed, as well as the pressure and the temperature. This ensemble is referred to as the isothermal-isobaric ensemble. The trial moves for the entities are equal to the NVT case in subsection 4.3.2.

Furthermore, the vectors $U$ and $V$ defining the simulation box size and shape - shown in Figure 3.1 - are subject to trial moves. The trial move is similar to the one for the entities of the system: The ends of both vectors are displaced to a randomly chosen position inside a square box defined by the stepsize. Then, $U$ and $V$ are rescaled, i.e. rotated, so that $V$ only has a component in the $\mathrm{x}$ direction [66]. If the energy of the system is smaller after the trial move the algorithm accepts the move. If not, the algorithm accepts the move with an acceptance probability according to what is sometimes referred to as the pseudo Boltzmann weight [72]:

$$
A\left(\mu_{U V} \rightarrow \nu_{U V}\right)=e^{-\beta\left(\left(E_{\nu}-E_{\mu}\right)+\tau\left(A_{\nu}-A_{\mu}\right)\right)+N \cdot \ln \left(A_{\nu} / A_{\mu}\right)},
$$

where $A_{\mu}$ is the area before and $A_{\nu}$ the area after the trial step. This change in volume entails a rescaling of the coordinates of the particles in the system. 


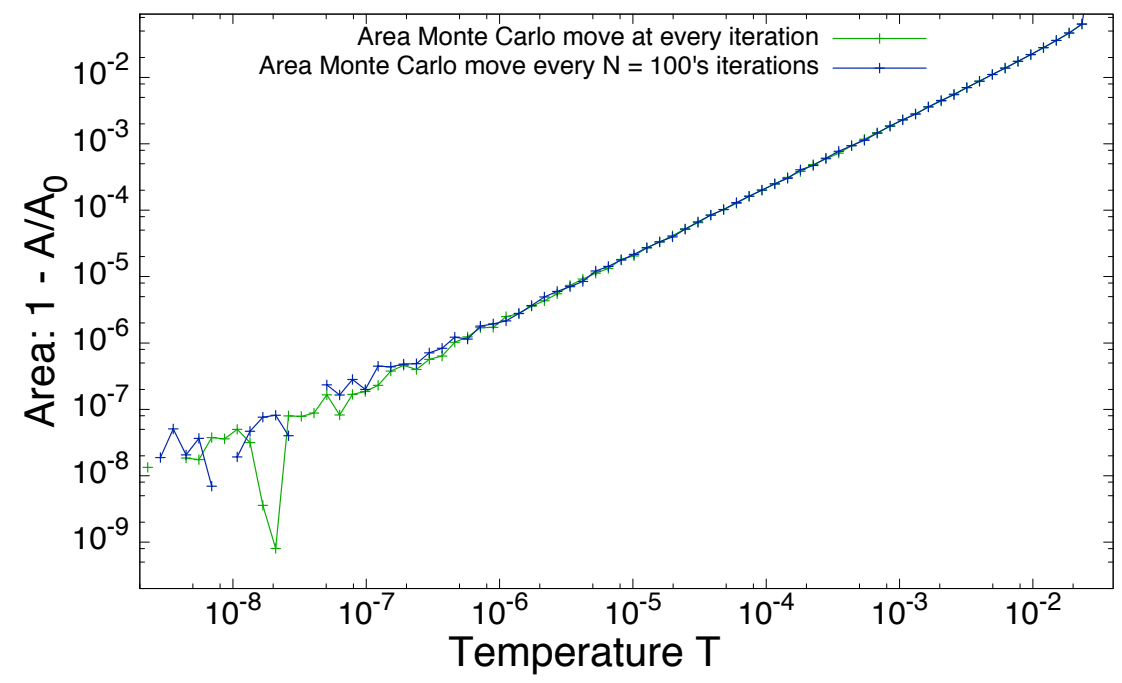

Figure 4.2: Comparison on how frequently the area needs to be adapted compared to moves on the vertices in a NpT-simulation of a spring network. The area moves cost computation time on the order of $N$ so they should be used sparingly. The choice of conducting area trial moves on every $N^{\prime}$ th step is supported by the comparison shown here. Furthermore the data on pressure, which are not explicitly shown here, tell that this choice accomplishes the applied external pressure - it is zero in the shown case. $z=4.7400, N=100, \tau=0.0$

This variable transformation has to be corrected in the partition function when integrating over the volume [73].

The trial moves for the vectors enclosing the system are performed after on average $N$ trial moves of spheres or vertices. Area trial moves are computationally costly as they involve adapting all $N$ vertices to the newly defined simulation box and calculating the energy for all the springs in the system. In 4.2 we can see that the choice of area moves after $N$ vertex steps is sufficient for a large regime of temperatures.

Thus, first the algorithm tries to relieve strain among all the entities of the system. Then, in addition it tries to reduce the total energy further by changing the system's area. The virial pressure in the system should then fluctuate around the applied external pressure: $p=\tau$. In Figure 4.3 this is verified. 


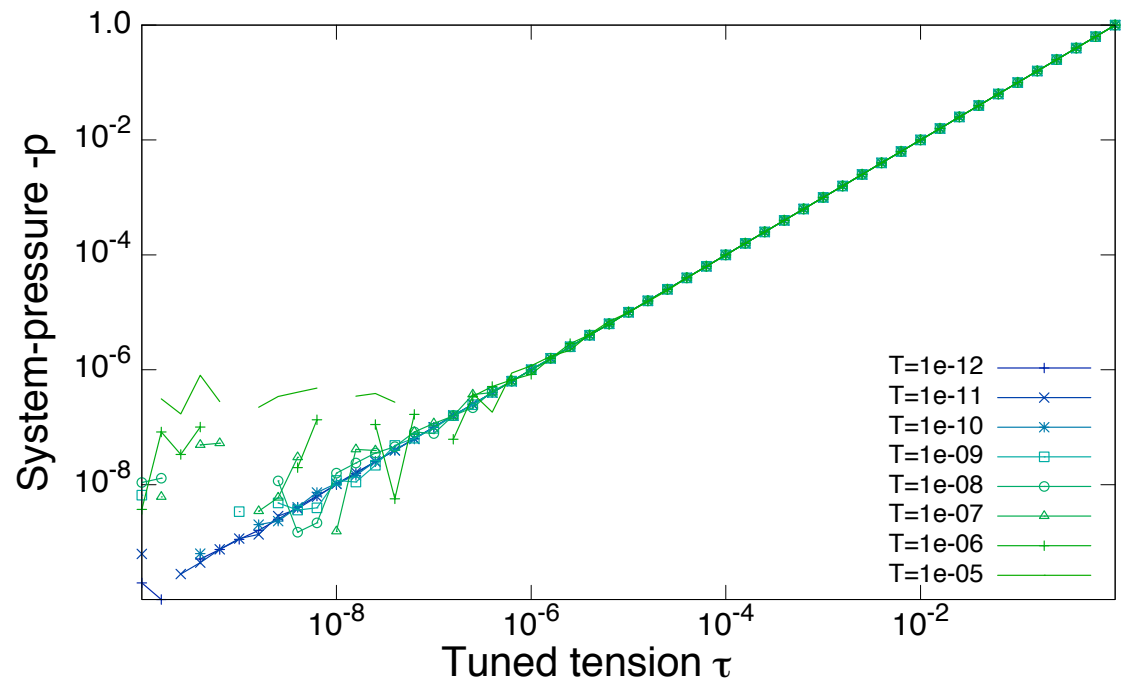

Figure 4.3: The system's pressure in the NpT simulation is equal to the tuned external tension $\tau$. For a system of $N=100, z=4.74$ and a wealth of temperatures $T$

\subsubsection{NpT simulation of spring networks}

The simulation of spring networks at fixed pressure is a particularly important case for the research presented here. It should be illustrated in greater detail.

In Figure 4.4 we try to illustrate the simulation dynamics induced by temperature in a snapshot.

In Figure 4.5 the collapse [74] of a spring network is shown. We speak of collapse when vertices cross other bonds, which leads to an ongoing folding of the structure. Due to the tendency to contract, eventually in this case the volume will vanish.

The snapshots of the collapsing spring network show that in this regime the simulation presented here is not meaningful any more: springs cross each other wildly because volume is not excluded. The model is not suitable to give results of interest at high temperatures which are associated with the collapsed region. Yet, this is sufficient because the subject of this study is to consider effects of rather low temperatures and to consider the solid phase.

Still, it is interesting to investigate with which parameters the collapse begins to set in. This is looked at in chapter 7 . 


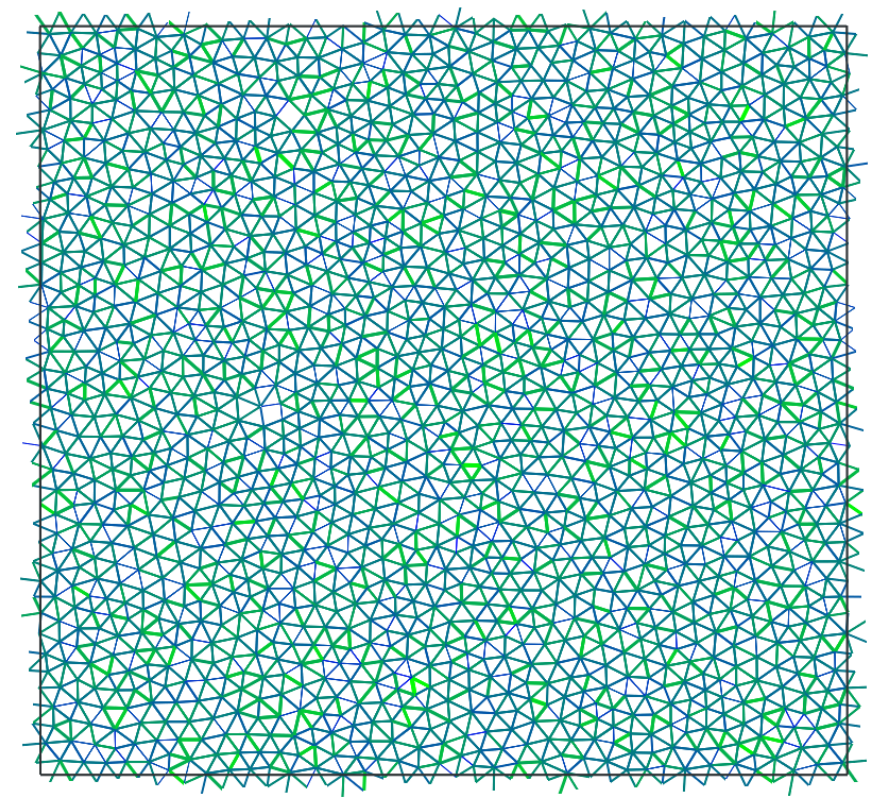

Figure 4.4: Snapshot of spring network excitations due to temperature. The thickness of lines is proportional to the relative length of the spring compared to its balanced length $s_{0}$. Thin lines indicate stretched springs, thick lines compressed springs. Colours range from bright blue to bright green. Blue denotes springs which are more stretched than the average in the system, while green shows springs which are more compressed than the average in the system. The system in this example is highly coordinated $z=5.94$ with $N=1600$, $T=10^{-5}, \tau=0.0$. 
4 Methods
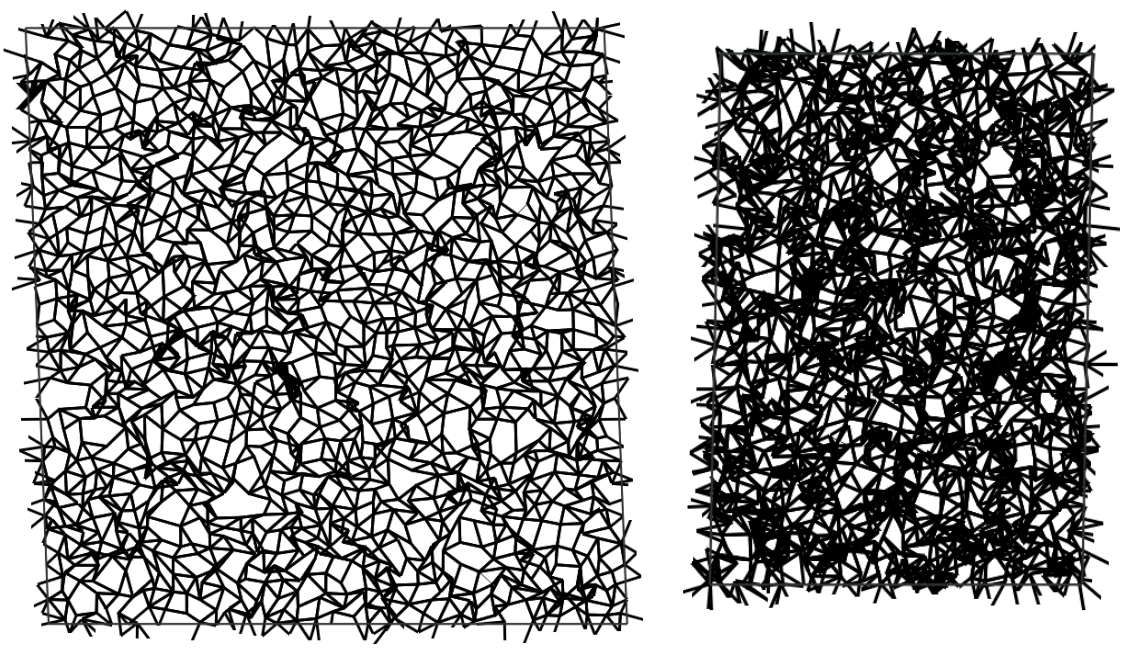

Figure 4.5: Collapse of the same spring network at two simulation times. The left picture shows the beginning of the collapse with the characteristic folding of some springs in certain regions. In the right picture the collapse is more advanced and the area of the system has decreased strongly. This effect is seen because volume is not excluded in our simulation and because it is not suited to model the behaviour at high temperatures. In this work we are interested in the effect of rather low temperatures and in where the phase transition to the collapse happens. The collapsed phase itself is not part of this work as the model we are using is not meaningful in a physical way any more in this regime. $z=4.0013, N=1600, T=10^{-2}, \tau=0.0$. 


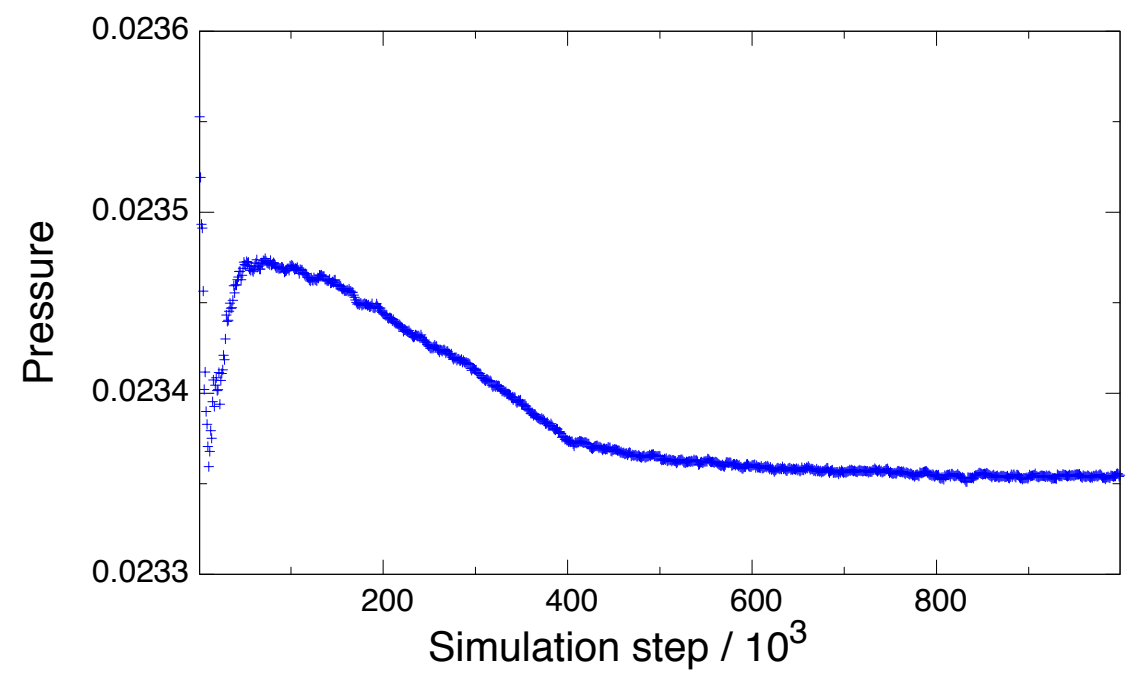

Figure 4.6: Pressure in a NVT simulation of a packing of spheres with initial equilibration because of initial minor rearrangements. Packing fraction $\phi=0.89(z=4.7400), N=100, T=10^{-9}$.

\subsubsection{Averaging}

We have set the acceptance probability according to the Metropolis algorithm 4.5 and we have made this choice so that our system moves through phase space sampling energies with probabilities stemming from the Boltzmann distribution. Thus, we can simply average the observable $o$ we are interested in over simulation steps $J$ to get an estimate for it in equilibrium:

$$
\hat{o}=\frac{1}{J} \sum_{j=1}^{J} o_{j}
$$

where $o_{j}$ is the value of observable $o$ at step $j$. Usually we do not measure $o$ at every single simulation time step as it involves extra computation.

Furthermore, successive steps that are closely together in the Markov chain are strongly correlated. Thus $J$ is in practice a small subset of all simulation steps performed and chosen so that correlations among steps are avoided as well as possible. A typical picture of the measurement of pressure in our system looks, for instance, as shown in Figure 4.6.

It must also be noted that we trim away a fraction of steps at the beginning which do not contribute to the set $J$ as the system is still in the process of equilibration, 


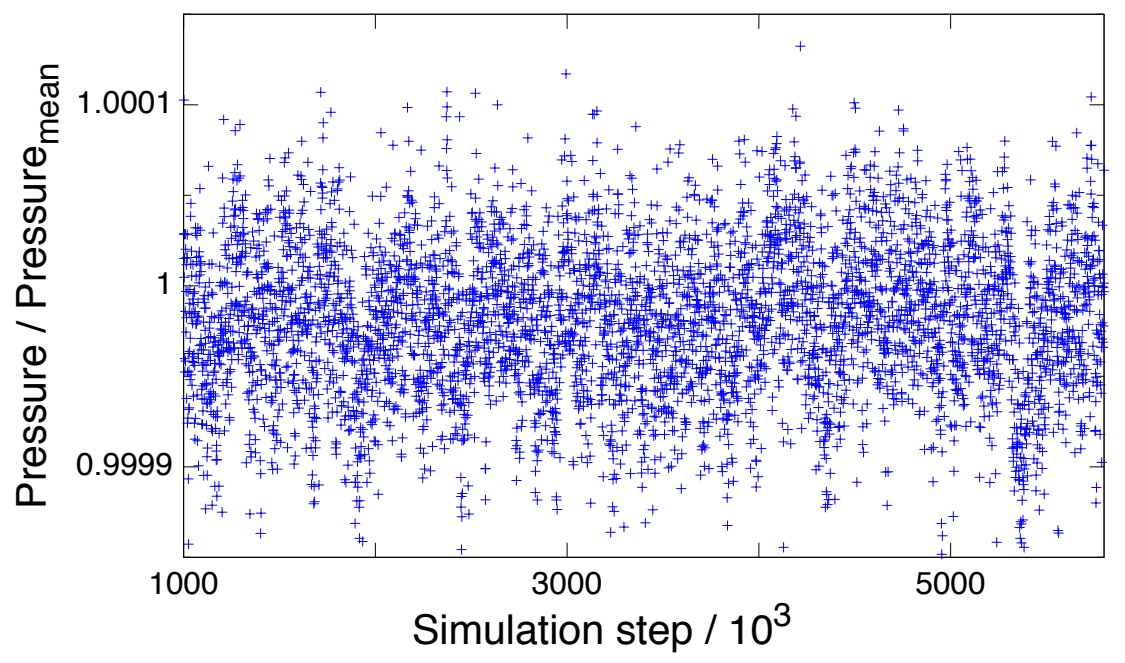

Figure 4.7: Pressure in a NVT simulation of a packing of spheres at equilibrium. Packing fraction $\phi=0.89(z=4.74), N=100, T=$ $10^{-9}$.

see 4.6. A typical timetrace of the NVT simulation of sphere-packings is shown in Figure 4.6, which shows the pressure fluctuations in equilibrium.

In Figure 4.8 a typical trace of the area measurement over simulation steps for the NpT ensemble is shown. Figure 4.9 shows the distribution of values for the observable area. Also there the burn-in or equilibration time at the beginning is cut and not used for the measurement of the average [75]. 


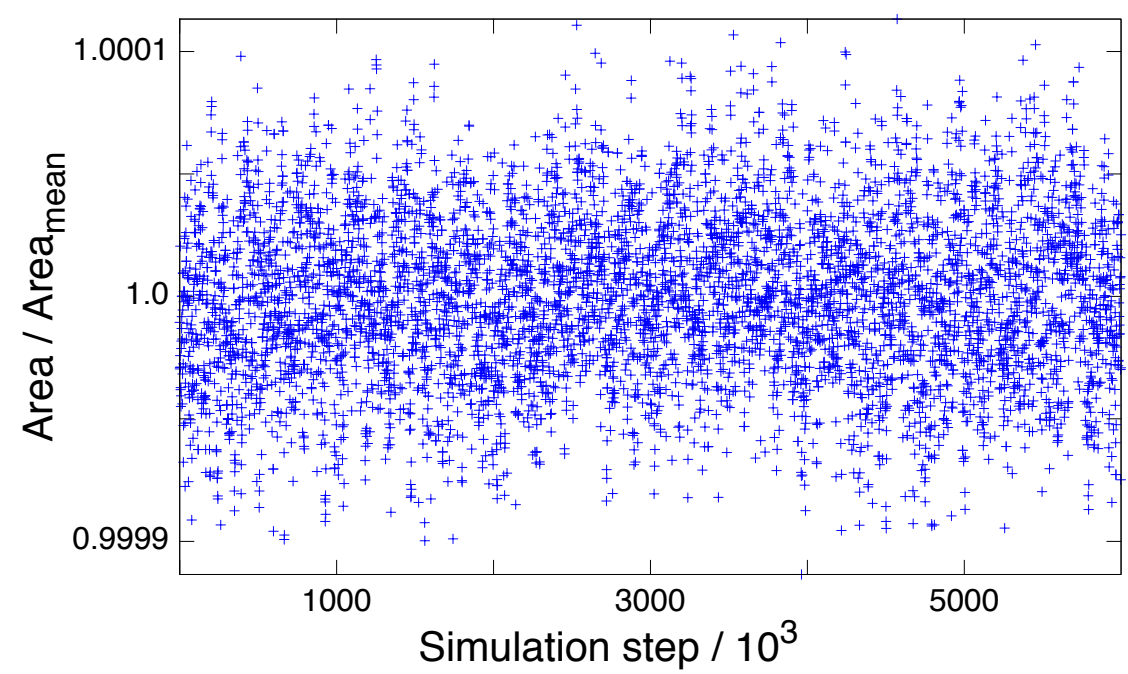

Figure 4.8: Area in a NpT simulation of a spring network at equilibrium. Initial equilibration is taken into account, $z=4.74, N=100, T=$ $10^{-8}, \tau=0.0$

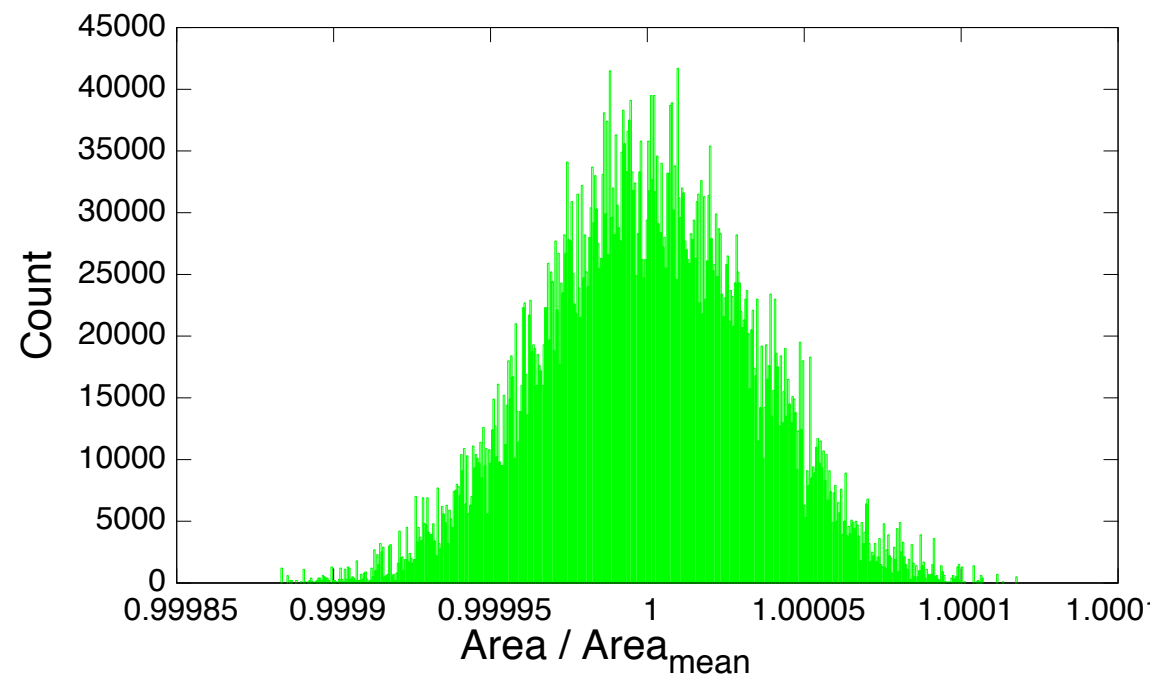

Figure 4.9: Histogram of area in a NpT simulation of a spring network at equilibrium. We can assume that the process obeys Gaussian statistics [76]. Initial equilibration is taken into account, $4.74, N=$ $100, T=10^{-8}, \tau=0.0$

Identifiying when a system is completely equilibrated is difficult to almost impossible to do rigorously. However, there are some hints: 


\section{Methods}

In Figure 4.6 we see that after some initial large changes in pressure a certain value is approached. This is probably the equilibrium we are looking for and the system's pressure in this case will fluctuate around the average equilibrium pressure. A second simulation with a different initial random seed would eventually reach the same average value. This way we know that the algorithm did not get stuck in a local energy minimum.

For relatively high temperatures, in which phase space exploration is dense, equilibration is usually not a problem in the simulations conducted: the temperature allows to overcome energy barriers easily during the simulation steps given.

For very low temperatures, the initial conditions of the system have a larger impact because the slow simulation dynamics take longer to reach equilibrium values. Hence, we have to increase the equilibration time. Yet it is hard to assert how many steps of heating the system are enough. The more simulation steps we can cut away at the beginnning the sharper the result. However, we need many measurements to get decent values for the observables of the system and simulation time has to be spent well. In practice we can look at the fluctuations of results at temperatures close to each other in order to identify down to which temperature we can trust the data. In packings we find glassy dynamics and ageing, hence we might never really a stationary state.

\subsubsection{Independent states and estimation of errors}

In order to find out how many simulation steps are needed so that two measurements are considered uncorrelated we look at the autocorrelation function. It is a simple measure for finding repeating patterns in a time series [69]:

$$
\begin{aligned}
& \text { Autocorrelation }(\mathrm{j})=\frac{1}{J-j} \sum_{j^{\prime}=0}^{J-j} o\left(j^{\prime}\right) o\left(j^{\prime}+j\right)- \\
& \qquad \frac{1}{J-j} \sum_{j^{\prime}=0}^{J-j} o\left(j^{\prime}\right) \cdot \frac{1}{J-j} \sum_{j^{\prime}=0}^{J-j} o\left(j^{\prime}+j\right),
\end{aligned}
$$

where $j$ denotes the time step in the set $J$ of the total number of measurements, $o(j)$ is the value of the observable we are considering at simulation time $j$. Using the autocorrelation in this form presupposes that the system is equilibrated and stationary.

In Figure 4.10 the autocorrelation is shown at two temperatures. Note that a change in area happens on average at every $N^{\text {th }}$ time step. 


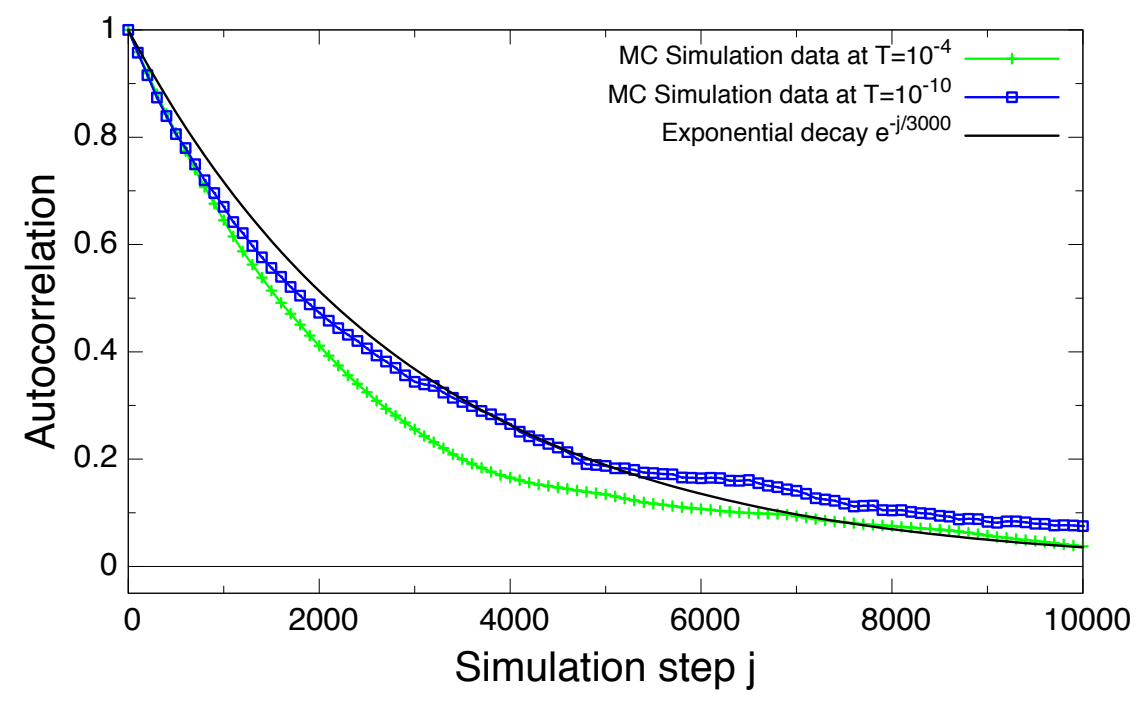

Figure 4.10: Autocorrelation of area after equilibration of a spring network at two temperatures. The autocorrelation decays with increasing simulation steps as it should. For the lower temperature the decay is slightly slower. The decay is expected to be exponential and on the order of $j_{d}=3000$ timesteps for reaching $1 / e \approx 0.37$. As a rule of thumb $2 j_{d} \approx 5000$ steps should at least be between two measurements of the area for this network and in this temperature range. $z=4.74, \tau=0.0, N=100$. 


\section{Methods}

The autocorrelation should decay like $\propto e^{-j / j_{d}}$ [69], which yields a timescale $j_{d}$ for the decay of correlations.

The correct standard deviation for the average of an observable is [77]:

$$
\sigma=\sqrt{\frac{1+2 j_{d} / \Delta j}{J-1}\left(\hat{o}^{2}-\hat{o}^{2}\right)}
$$

where $\Delta j$ is the interval between two measurements. For large $J$ we can thus use the standard error:

$$
\sigma \approx \sqrt{\frac{1}{J-1}\left(\hat{o}^{2}-\hat{o}^{2}\right)}
$$

as long as there are at least two correlation times between two measurements: $\Delta j>2 j_{d}$.

If we are taking more steps that are correlated, we have to correct $J$ in (4.10) to the number of actually uncorrelated steps. In other words: it is the number of uncorrelated observations that counts rather than the total number of observations [75]. One practical problem is that it is hard to know beforehand what the autocorrelation time will be, so we will choose the number of iterations to be sufficient, based on the knowledge of previous simulations.

The standard deviation amounts to the statistical error of a simulation run. The systematic error of the simulation is much harder to estimate. It is connected to the way the simulation is choosing its steps and to properties such as the equilibration time. In 4.3.5 the difficulties in knowing equilibration times were already discussed. For the systematic error this means that it is very hard to estimate, too. For the results of the simulations conducted we can see on the basis of consistency when values for low temperatures get out of hand compared to higher temperatures, even though statistical errors might be small.

In Figure 4.11 the measured average area is shown over a regime of relatively low temperatures. 


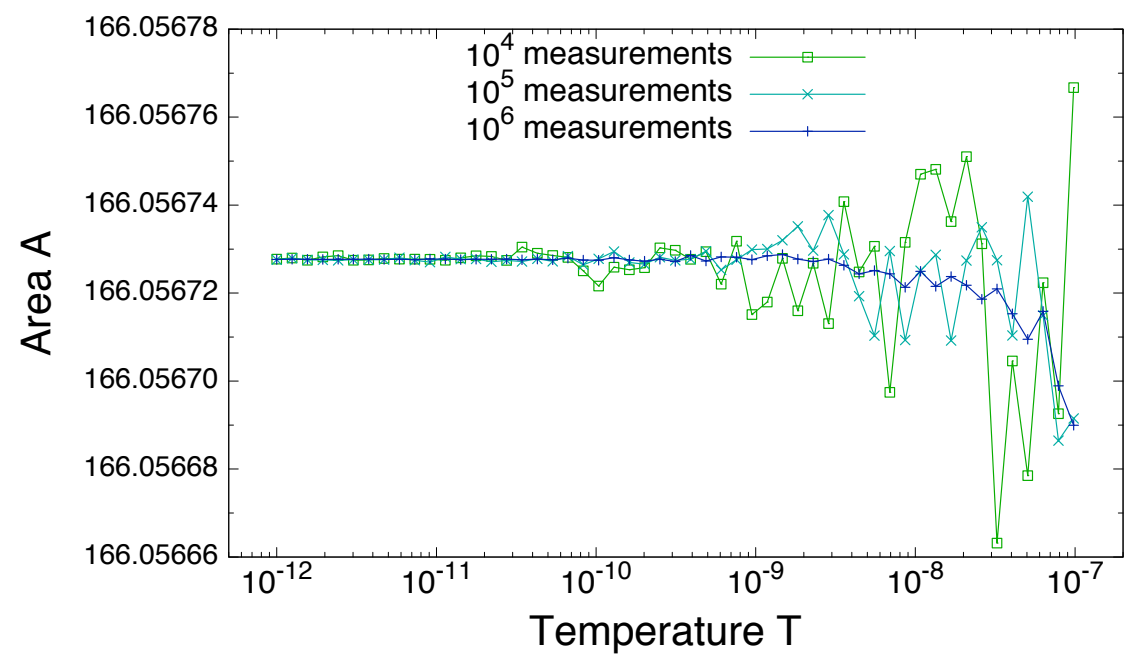

Figure 4.11: Fixed pressure Monte Carlo simulation of spring network with coordination $z=4.9495$ for number of measurements. Error bars for the statistical errors are smaller than the symbols for a data point. Increasing the number of measurements while having the same equilibration time improves the quality of the area-overtemperature graph significantly.

It can be seen that increasing the number of measurements while using the same equilibration time improves the quality of the final result significantly. This is the case even though the statistical error is small compared to the fluctuations inside the graph and the distance between two measurements is the same, i.e. $\Delta j>2 j_{d}$.

\subsubsection{Optimisations - Neighbour lists}

In scientific simulations it is essential to be efficient with respect to the given computational - both processing and spatial - resources. This may determine if a result can be obtained in a humanly meaningful time and, thus, if certain phenomena are observed at all. As Newman and Barkema [69] point out it is rarely the most efficient approach to just program the Monte Carlo Metropolis algorithm. Small deliberations can improve the runtime by orders of magnitude.

A common way to reduce the computational complexity of a system with interacting particles in a box is to employ a neighbour or Verlet list [73].

In the case of spring networks this optimisation is not necessary or, alas, trivial because the fixed connectivity of the vertices naturally defines a neighbour list. 


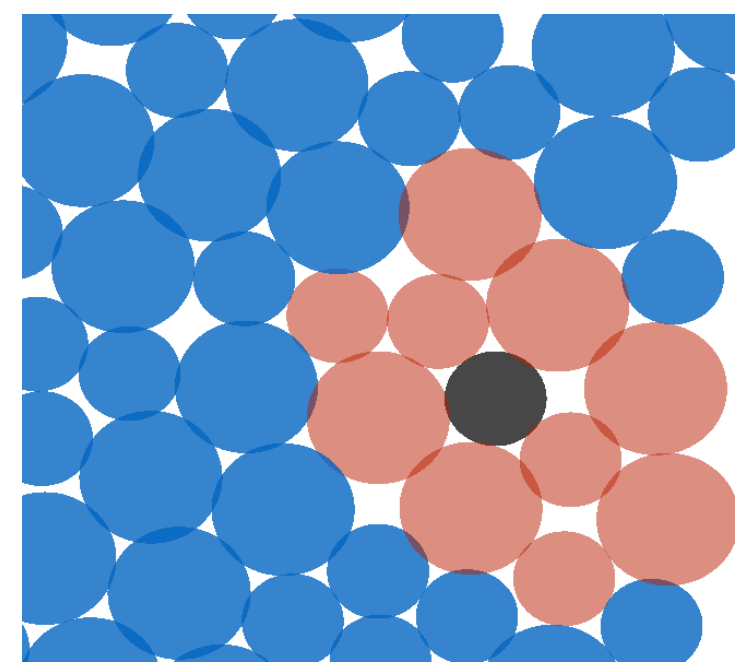

Figure 4.12: Neighbourhood of a disc in the packing. Neighbourhood of given discrete length for a disc, marked in black, in the packing. Its neighbours are marked in red. Discs which will not be in the neighbour list are coloured in blue. Blue discs will not be evaluated in a trial step, which is unproblematic due to the short-ranged interactions. The size of the neighbourhood is 10 .

This means that only the up to six springs connected to neighbouring vertices must be evaluated in order to get the change in energy of the Hamiltonian of the system. Thus, the computation cost is of order $O(N)$ for a trial move of every vertex.

In the case of thermalized packings the neighbour relations between spheres may change. Naively speaking, this means evaluating all possible interactions between spheres in the box which would drive the computation cost to $O\left(N^{2}\right)$. As a remedy a list of neighbours for each sphere is kept and only interactions between the sphere and the sphere in its neighbour list are evaluated. The neighbour list is pictured in Figure 4.12.

The mean free path length of the system determines how often the neighbour list has to be updated. In a gas for instance, the neighbour list needs to be updated frequently with respect to the possible displacement of a particle in one step since their environment(the neighbouring particles) changes rapidly over time. This extra effort in programming was saved and a version which can be implemented with low programming complexity and which is just right for our case was chosen. In the simulations conducted in this work it is enough to keep a constant neighbour list initialized in the beginning of the simulation. This is because in our jammed system the interactions do not change that fast and 
we can use such a "simple" implementation of neighbour lists and only update those when necessary at a long time interval. This is motivated by the fact that movements of spheres are rather small and rearrangements only happen at high temperatures. The length of the neighbour list is tuned to the temperature of the simulation and checks to confirm that the neighbour list is still sufficient are performed at certain intervals. For high temperatures we simply use the whole system as a neighbour list, which is sufficient as we do not need many steps to get enough uncorrelated steps for measurements.

\subsubsection{Optimisations - Choice of stepsize}

An adequate choice of the stepsize reduces the simulation effort of our model system drastically. We have to ask ourselves what a good stepsize is and how to find it. The acceptance rate is an important measure for Monte Carlo simulations in order to understand if parameters are correctly chosen. The acceptance rate is defined as:

$$
\text { Acceptance rate }=\frac{\text { Steps accepted }}{\text { Total number of MC steps }}
$$

The acceptance rate is a direct result of the choice of a certain stepsize [76]. In our case, having a high acceptance rate means that each sphere or vertex in our system is covering its vicinity with many small successful steps. Even though not many steps are wasted this means that for a given finite number of steps we only cover a small area around each sphere. Moreover, the coverage in phase space of our system is probably not optimal either. On the other hand, a low acceptance rate means that most of the suggested steps are dismissed by the Monte Carlo criterion. This is not an optimal situation either because it will result in a series of strongly correlated steps.

As it is not apparent how to fix the acceptance rate, we introduce another measure which we will call total movement. The total movement is simply the sum of steps accepted of all vertices added up over a certain simulation time interval:

$$
\text { Total movement }=\sum_{j}^{J} \sum_{i}^{N}\left|\delta x_{i}\right|+\left|\delta y_{i}\right|
$$

where $J$ is the number of simulation steps for the chosen interval, $\delta x$ the displacement in $x$-direction, $\delta y$ the displacement in $y$-direction and $N$ is the number of entities (spheres or vertices) in our system. This measure should not be mistaken for the mean square displacement. In general, the total movement should be 
4 Methods

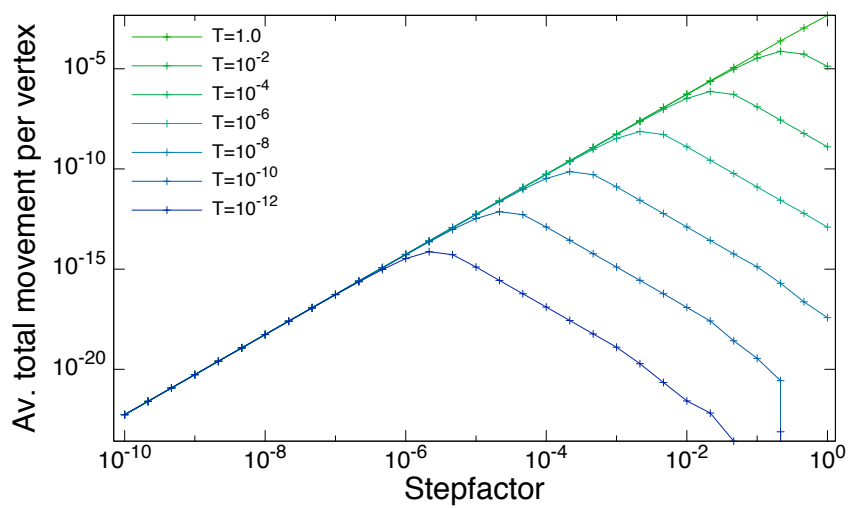

Figure 4.13: Total movement per vertex as a measure of the efficiency of phase space exploration for different temperatures over a range of stepsizes. The best stepsize in terms of computational efficiency depends on the temperature of the simulation for a given network. The described algorithm finds the best stepsize at all times and makes use of the convexity of the total movement function over stepsize. NpT simulation of spring network with coordination $z=$ 4.86 with $\tau=0.0$.

divided by computing time. In our case, however, the computing time does not depend on accepted or rejected moves or the magnitude of trial step.

The goal is to maximize this measure with the choice of the stepsize. The higher the total movement, the larger the environment each vertex or sphere is exploring with respect to a given amount of Monte Carlo steps. The total number of the Monte Carlo steps will include dismissed as well as accepted moves because we are interested in the efficient exploration of phase space volume.

Special care, however, has to be taken of rattlers so that this measure can be a meaningful heuristic for the efficiency of the simulation. They should be excluded, since if this is not done the rattlers will explore their free volume and drive the stepsize to a value which optimises the exploration of the free volume of the rattlers. A choice of such a stepsize, however, is not optimal for the non-rattlers.

It seems most simple and straightforward to implement the search for an optimal stepsize - as defined by our total movement measure - by implementing a finite state machine. We utilize the fact that the function of the total movement over stepsize is convex for an infinitesimal number of measurements $J$, see Figure 4.13 as an illustration for a finite number of steps.

This can be understood from the argument that for low stepsizes a small amount 
of phase space is explored. If we increase the step size the degree of exploration measured by the total movement - is increasing. At the other end of the spectrum, for very large stepsizes almost no movement and thus exploration of phase space is happening as steps are neglected by our Monte Carlo criterion. In between those two ends there has to be a maximum of the total movement.

The algorithm to maximize the phase space exploration is:

1. Initialize a vector $S$ of stepsizes which will be evaluated in the following. Go to state 2 .

2. Record values with the chosen stepsize $s$ for $J_{t r y}$ iterations. Do this for all stepsizes in $S$.

Go to 3 .

3. Evaluate best stepsize $s_{b}$ out of $S$ with respect to the highest total movement and run for this stepsize a number of iterations $J_{\text {run }}>>J_{\text {try }}$.

Go to 1 .

The application of this measure to fixed step sizes for simulation runs at different temperatures can be seen in Figure 4.13. The described algorithm finds the best stepsize defined by generating the highest total movement at all times.

\subsubsection{Observables}

Now we will briefly define the observables of the system and how they were obtained within the simulation.

\section{Area}

The area of the system is defined by the encompassing vectors $U$ and $V$, as defined in Figure 3.1. The area of the parallelogram is then measured straightforwardly:

$$
A=U_{x} \cdot V_{y}
$$

where $U_{x}$ is the component of $U$ in the $x$ direction and $V_{y}$ the component of $V$ in the $y$ direction. Note that $V$ is aligned with the $\mathrm{y}$-Axis.

\section{Pressure}

We measure the virial terms of the pressure, i.e. neglect pushes with the walls of the system of order $N \cdot T / A$. These contributions are small compared to the 


\section{Methods}

virial terms in the temperature ranges we operate. The pressure is defined as a dimensionless quantity:

$$
p=\frac{1}{k_{s p} \cdot d \cdot A} \sum_{i j}^{N} s_{i j} \cdot f_{i j},
$$

where the sum goes over all pairs $i j$ with distance $s_{i j}$ among them, the factor $d$ indicates the dimension of the system and the force $f_{i j}$ between entities $i$ and $j$ being:

$$
f_{i j}=k_{s p} \cdot\left(s_{0}-s_{i j}\right)
$$

The equilibrium length of the spring is $s_{0}$ or in the case of packings the sum of radii $r_{i}+r_{j}$ of both spheres. For $s_{i j}>r_{i}+r_{j}$ the force between the two spheres vanishes. The constant factor $k_{s p}$ of the potential - i.e. the spring constant - is generally set to unity.

\section{Coordination}

Average coordination $z$ of a spring network is defined as:

$$
z=\frac{2 Z}{N-R}
$$

where $Z$ is the total number of springs in the network and $R$ the number of rattlers (the number of springs or spheres with $\leq 1$ contact, see Chapter 2.1).

For simulations of packings we measure the effective coordination number $z_{\text {eff }}$ in which $Z$ becomes the number of contacts at simulation time $t$. More details on coordination are dealt with in chapter 2 .

\section{Direct bulk measurement}

The bulk modulus can be measured directly by using its definiton $[78,79]$ :

$$
B=-A \frac{d p}{d A}=(1-\delta A) \frac{d p}{d \delta A}
$$

where $\delta A=1-A / A_{0}$ is the relative change of the area. This change can be conveniently used in order to probe the derivative in the equation. 


\section{Bulk modulus through linear response}

The bulk modulus can be measured through the fluctuations in the area with help of the fluctation dissipation theorem [80]. Its response to fluctuations of the area are the same as to a small applied force [73].

The bulk modulus $B$ is then [66]:

$$
B=T \frac{\langle A\rangle}{\left\langle\Delta A^{2}\right\rangle},
$$

where $\langle A\rangle$ is the average in area obtained through Monte Carlo sampling and $\left\langle\Delta A^{2}\right\rangle$ the corresponding variance.

Measuring the bulk directly through this method we have to run series of simulations for different values of fixed $\delta A$. Measuring the bulk via fluctuations we need to sample longer for one combination of $(T, z, \tau)$. There is no free lunch after all.

\section{Simple shear modulus through linear response}

The simple shear modulus [81] can be derived through the fluctuations in the defining vector $V$ of the volume's shape [66]:

$$
S=\frac{T}{\langle A\rangle}\left\langle\left(\frac{\langle V y\rangle}{V x}\right)^{2}\right\rangle=T\langle A\rangle^{-1}\left\langle V_{y}\right\rangle^{2}\left\langle V_{x}^{2}\right\rangle^{-1} .
$$

In Figure 3.1 the angle $\alpha=\tan \left(\frac{V y}{V x}\right)$.

\section{Localisation length}

We define the localisation length $L L$ of an entity $i$ in the system as:

$$
L L=<\left(r_{i}(t)-<r_{i}(t)>\right)^{2}>=\operatorname{Var}\left(r_{i}(t)\right)
$$

In principle one could calculate the variance directly from the movement of an entity, but this turned out to be unreliable and not stable to handle as global movements and rotations of the system have to be taken care of. Furthermore, using (4.20) directly means collecting the positions of $i$ first in order to compute the average and only then the variance. 


\section{Methods}

Instead of using the definition directly, the variance of the distance of two entities $i$ and $j$ was measured. If they are uncorrelated, this gives twice the localisation length:

$$
\begin{aligned}
\operatorname{Var}\left(r_{i}(t)-r_{j}(t)\right)= & <\left(r_{i}(t)-r_{j}(t)\right)^{2}>-<\left(r_{i}(t)-r_{j}(t)\right)>^{2} \\
= & <r_{i}(t)^{2}>+<r_{j}(t)^{2}>+2<r_{i}(t) r_{j}(t)>-<r_{i}(t)-r_{j}(t)>^{2} \\
\approx & <r_{i}(t)^{2}>-<r_{i}(t)>^{2}+<r_{j}(t)^{2}>-<r_{j}(t)>^{2} \\
\approx & 2 \cdot \operatorname{Var}\left(r_{i}(t)\right) \\
\approx & 2 \cdot L L
\end{aligned}
$$

In the second step we assume both entities to be uncorrelated. In the third step we use the fact that the localisation length does not depend on a specific entity.

To determine the localisation length of entities in the simulation box the following algorithm is used:

1. Choose a set of entities in the system

2. Record their distance

3. Calculate the average distance and its variance of each entity-pair of the selection

If we choose two vertices $i$ and $j$ which are far away enough away from each other, their movements over simulation time $t$ will not be correlated. Therefore the variance of their distance will give us twice the localisation length of one of the entities itself:

Figure 4.14 shows the variance in distance over mean distance.

It can be seen that a certain distance is needed to be safe that vertices are uncorrelated. Certainly this decay is stronger in a larger system, see Figure 4.15.

For our purposes it is enough to average over the variance in distance of the largest average distances. Divided by 2 and the average radius $(0.5+0.7) / 2=0.6$ squared this is the mean square displacement. 


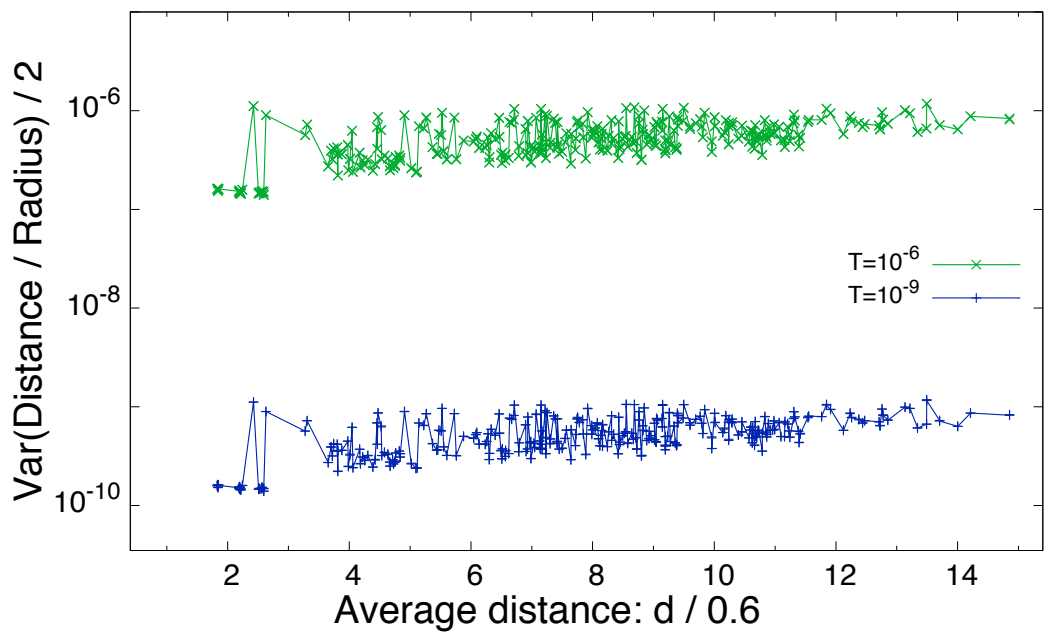

Figure 4.14: Variance of distance plotted over distance of vertices in a spring network. For a meaningful lengthscale, variance and average distance are corrected by the average radius of spheres in the initial packing configuration. In this figure and work this is: $(0.5+0.7) / 2=0.6$. The scaling on temperature can already be assumed from this plot - it will be discussed in section 5.4. $N=100$, $z=4.74$, Edge-length $L / 0.6 \approx 21.5$. Minimum image of two points with maximum distance ( $L / 2$ in $x$ and $y$ direction) is 15.2 . 


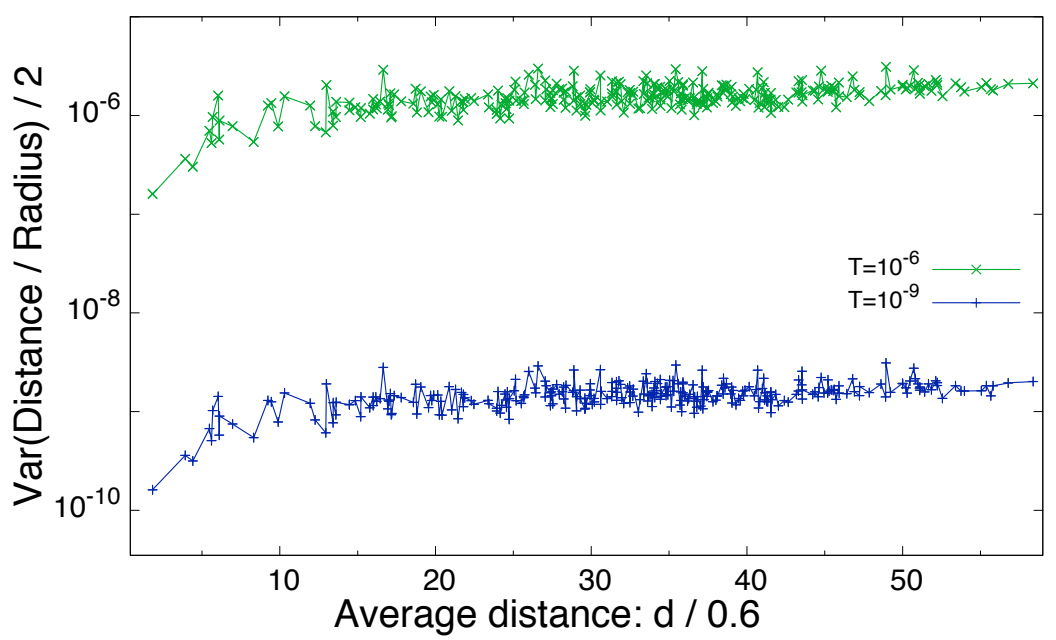

Figure 4.15: Variance of distance plotted over distance of vertices in a spring network with $N=1600$ vertices. In Figure 4.14 there is a cutoff in distance at around 15, due to the smaller system size. In this figure it can be seen that it is sufficient to estimate the mean square displacement through the variance in distance even in such a small system with $N=100 . \quad N=1600, z=4.39$, Edge-length L $/ 0.6 \approx$ 85.9. Minimum image of two points with maximum distance is 60.7 .

\subsection{Concluding remarks}

The simulations conducted in this work are marked by the problems which come up when doing computer experiments at low temperatures of glassy systems [82]. The limitation one has to work around here is that many states are blocked by large energy barriers, which are practically impossible to overcome when the temperature is low. This is known as ergodicity breaking.

Thus rearrangements disregarded in this research on jammed packings as we focus to sample the small movements consistent with the structure of the packing or spring network. In this trimmed state space ergodicity can be assumed. Still, the given scenario results in long equilibration because of the strong history dependence of the sampling.The optimisations employed were good enough for what we wanted to study over a large range of temperatures. Yet, there is a strong dependence on the implemented algorithm and randomness for these kinds of systems.

Possible improvements would be to employ more advanced Monte Carlo techniques like entropic sampling or simulated tempering. In the first method, state space is sampled with higher probability in regions where the density in state 
space is low. This is done because in glassy systems these phase space regions are not well sampled in importance sampling. This way the partition function is approximated, which can then be used to calculate all properties of the system at all temperatures. It has non-trivial aspects to it in programming and practice. In simulated tempering a second simulation at a higher temperature is conducted and in exchange with the actual simulation. The higher temperature of the second simulation helps to overcome energy barriers. 



\section{Thermally Activated Networks of Springs}

In this chapter we will present the results thermally activated networks of springs. The setting is clear: jammed materials exhibit ample intriguing phenomena already in the athermal case. Here, we want to investigate how the model sytem described in chapter 3 behaves when we turn on the heat. Further parameters are the varying connectivity and external pressure. Our main focus is on the resulting linear response. 


\subsection{Area development}

As explained in section 1.3, negative thermal expansion is an interesting phenomenon of certain usually amorphous materials. To investigate this effect for amorphous spring networks the area of the system is measured at different temperatures. In Figure 5.1 we can see the area development under temperature for a network with coordination $z=4.9495$ and $N=100$ vertices. The pressure of the simulated system network is fixed at zero. This is the $N p T$ ensemble, where the number of particles or vertices $N$, the pressure $p$ and the temperature $T-$ for each data point - are fixed.

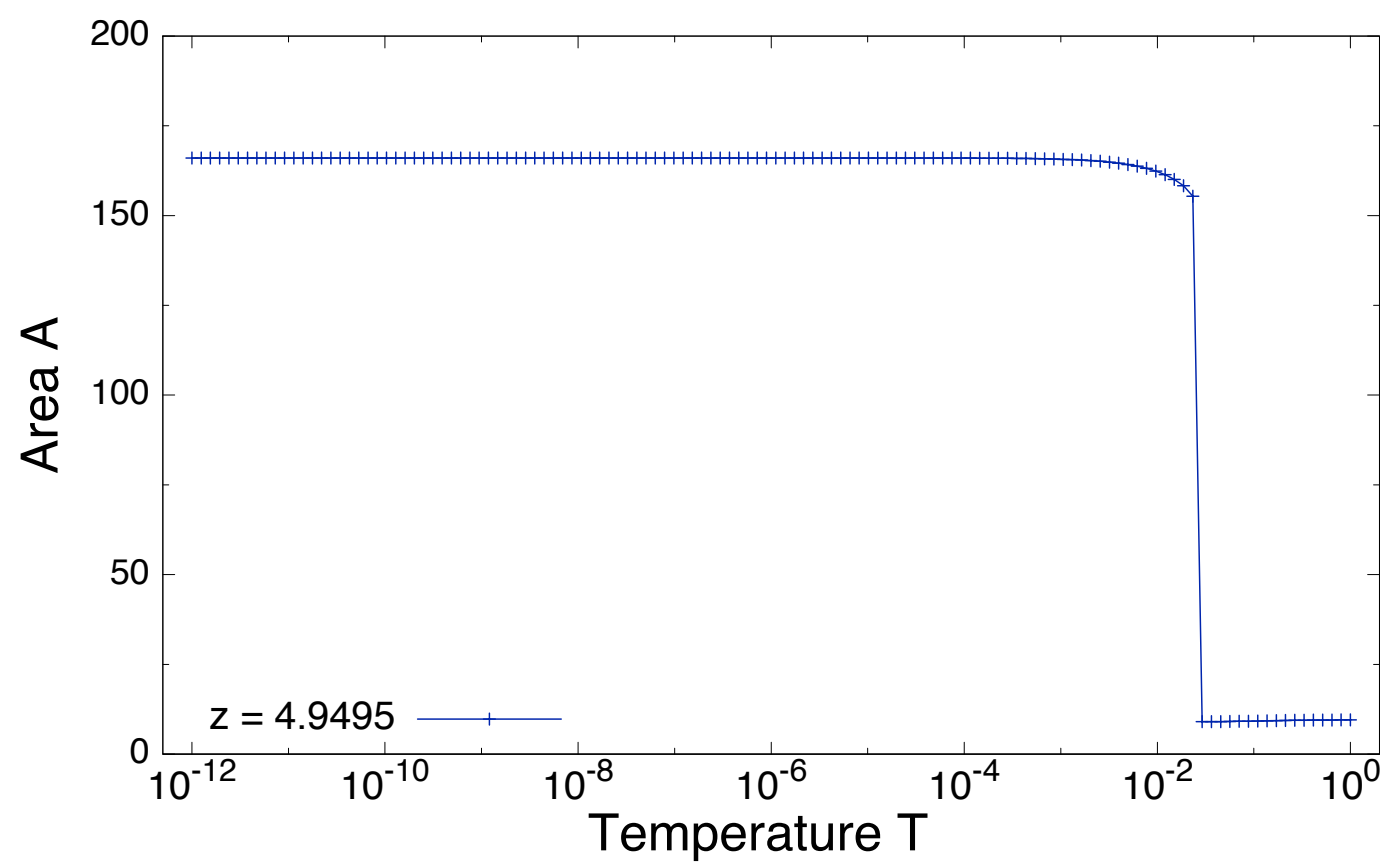

Figure 5.1: Fixed pressure Monte Carlo simulation of a spring network with coordination $z=4.9495$. The system contracts with increasing temperature up to a transition temperature $T_{c}$. For $T>T_{c}$ the spring network is collapsed. In the collapsed regime the model is not physically meaningful anymore. This regime will be discarded in the following. System size $N=100$.

The area development in Figure 5.1 shows two regimes: For very low temperatures up to approximately $T_{c}=2.9 \cdot 10^{-2}$ the area $A$ first looks constant on the linear scale and then decreases. This contraction is a first hallmark of negative thermal expansion. Then the system's area decreases rapidly and by orders of magnitude at $T_{c}$ and the system is found in a collapsed state. 
The main reason is, that the simulation model itself breaks down quite literally at this point. As volume is not excluded in the simulations, vertices go criss-cross - Figure 4.5 shows a snapshot of the situation - and the gathered data in this regime is not of a meaningful system any more. Yet, the model works as expected up to this transition.

The point of the transition will be of interest in 7 where the phase diagram of spring networks is discussed. The collapsed state itself, however, will not be discussed further in this work.

\subsubsection{Negative thermal expansion}

We will focus now on the contraction of the area with temperature. The general definition of the thermal expansion coefficient is [81]:

$$
\alpha_{V}=\frac{1}{V}\left(\frac{\partial V}{\partial T_{p}}\right)
$$

Applied to the two-dimensional model system we get:

$$
\alpha_{A}=\left.\frac{1}{A_{0}} \frac{\partial}{\partial T} A(T)\right|_{\Delta z, \tau}=-\frac{\partial}{\partial T}\left(1-\frac{A}{A_{0}}\right) .
$$

The term $1-A / A_{0}$ is the relative contraction, which compares the area to the area $A_{0}$ at zero temperature for the given values of $\Delta z$ and $\tau$. The relative contraction in the contracted regime is shown in Figure 5.2. 


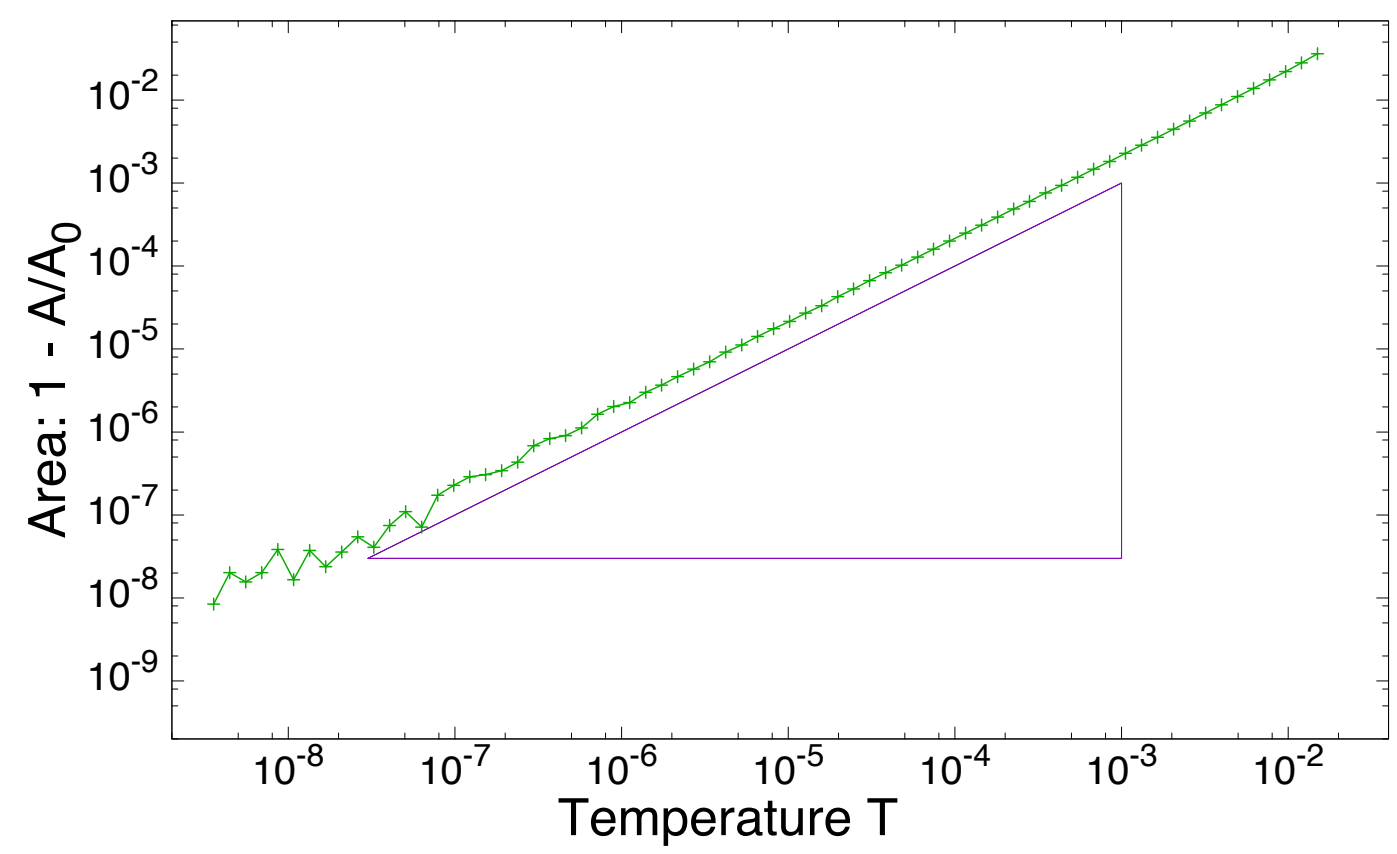

Figure 5.2: Spring network with coordination $z=4.9495$ as a function of temperature $\mathbf{T}$. The area is measured as the relative contraction $1-$ $A / A 0$. The triangle has a slope 1 proportional to the temperature. We see that the network contracts directly proportionally to temperature $T$ over at least five decades. System size $N=100$.

We see that the network contracts directly proportionally to temperature $T$ over at least five decades. The lower the temperature, the longer simulations are needed in order to get precise values. That is why the line is not smooth at low temperatures. This was explained in detail in Chapter 4. Thus we find:

$$
-\alpha_{V} \sim T
$$

Next, it has to be checked how this result depends on network properties. The coordination number clearly is a determining quantity for spring networks as it is directly connected to its mechanical stability and mode spectrum [29]. Figure 5.3 shows a variety of networks of different coordination.

Firstly, it can be seen that a higher coordination number accounts for a stiffer network, i.e. with a lower contraction at a given temperature. Furthermore, it can be seen that the temperature $T_{c}$ at which the collapse is happening increases with coordination $z$. Most importantly, we find that the negative thermal expansion is 


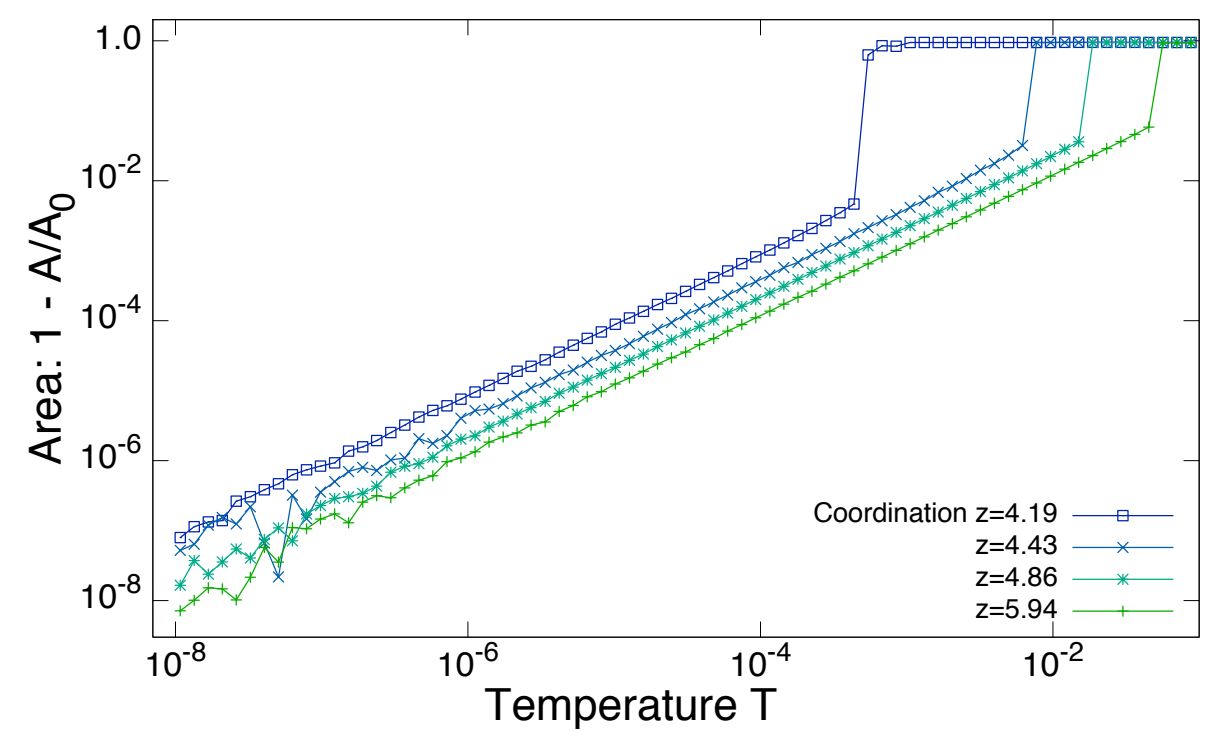

Figure 5.3: Fixed pressure Monte Carlo simulation of spring networks with different coordination numbers $z$. A network becomes stiffer and more resilient to temperature with increasing connectivity. The linear scaling with temperature holds for different coordination numbers $z$ over many decades. System size $N=100$.

proportional to $\mathrm{T}$ independently of the coordination $z$ : spring networks contract with temperature.

\subsubsection{Effective coordination number}

In Figure 5.4 we show the average effective coordination $z_{\text {eff }}$ for different spring networks as a function of temperature. Measuring effective coordination is done by taking a snapshot of the network at a simulation time to measure the coordination of the corresponding packing of spheres. In other words, each vertex is exchanged through its corresponding sphere with radius $R_{1}$ or $R_{2}$ and then (2.1) is used. This quantity is interesting because it provides an understanding of how strong the movement of the vertices is due to the heat. Furthermore, the effective coordination number is a useful order parameter to discriminate the contracted from the collapsed regime in the case of spring networks. This will be important in Chapter 7 where we consider the phase diagram of these spring networks. It will be especially important when we later consider sphere packings in Chapter 8 as there exists no fixed neighbour connection for spheres. 


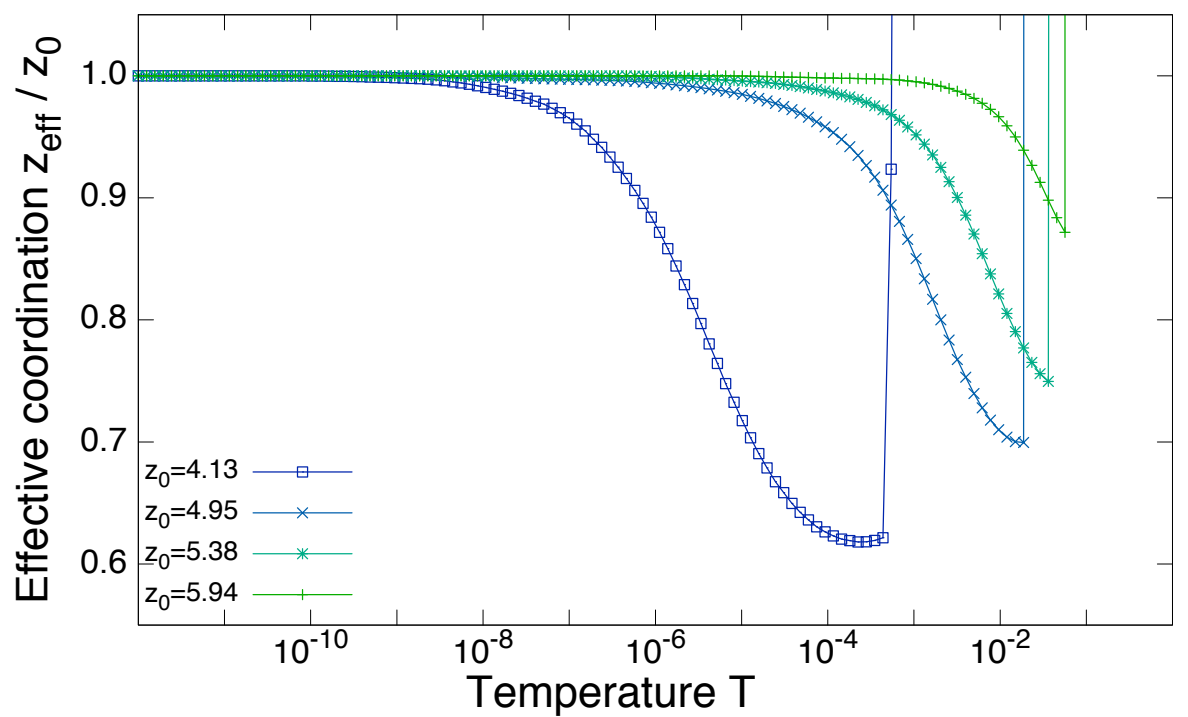

Figure 5.4: Average effective coordination $z_{\text {eff }}$ for spring networks with different coordination numbers $z$. The effective coordination number measures the coordination of the network at one point in simulation time as if it was a packing of spheres. The shown values are the simulation time and thus ensemble averages for given temperature and connectivity of the network. This quantity is interesting because it provides an understanding of how strong the movement of the vertices is due to the heat. On average the number of contacts decreases with temperature. 


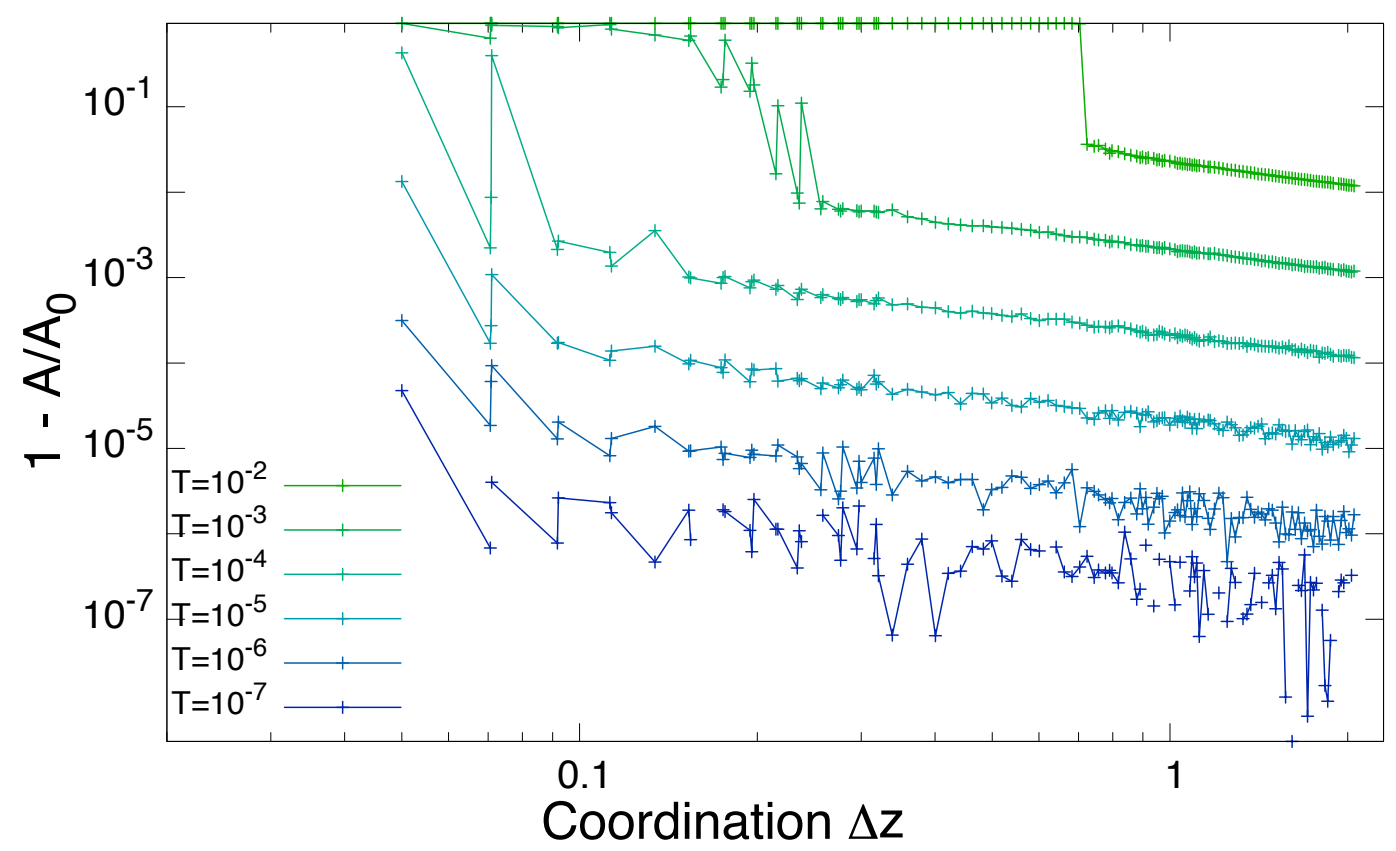

Figure 5.5: Relative contraction of spring networks for different temperatures over the distance in coordination $\Delta z$ from the isostatic point. Low coordination numbers and high temperature favor collapse of a spring network. Furthermore, networks contract with diminishing distance from the isostatic point. Low temperatures are computationally costly to simulate, hence the more wiggly data.

\subsubsection{Dependence on coordination number}

The dependence on the connectivity of spring networks will now be investigated further. In Figure 5.5 we plot the relative contraction as a function of the distance from the isostatic coordination number $\Delta z$.

In Figure 5.5 we see that the lower the coordination numbers the lower the critical temperature $T_{c}$ above which the spring networks collapse. Furthermore, networks contract with diminishing distance to the isostatic point. This is not surprising as a lower coordination is a mark of lower mechanical stability and should result in contraction. The wiggly data for low temperatures again stems from the computational cost to simulate in this parameter range.

The linear dependence of the reduced volume on temperature was already established before, thus it can be scaled out. This is done in Figure 5.6. 


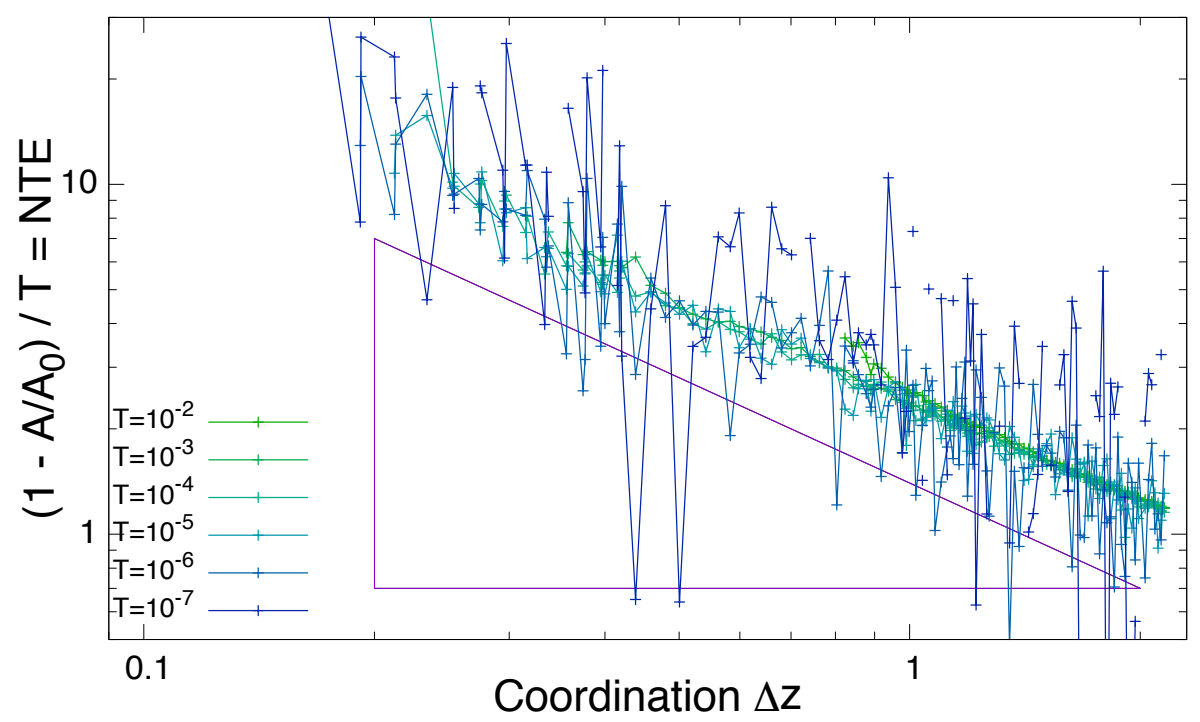

Figure 5.6: Fixed pressure Monte Carlo simulation of spring networks with different coordination numbers $z$. With increasing network connectivity they become stiffer and more resilient to temperature. Linear scaling with temperature holds for different coordination numbers $z$ for 1 decade. The triangle has a slope $\Delta z^{-1}$.

The following dependence is found:

$$
\frac{1}{T}\left(1-\frac{A}{A_{0}}\right) \sim \frac{1}{\Delta z}
$$

Due to the linear dependence of the area on temperature the negative thermal expansion NTE coefficient becomes:

$$
\mathrm{NTE}=-\alpha_{A}=\frac{\partial}{\partial T}\left(1-\frac{A}{A_{0}}\right) \sim \frac{1}{\Delta z} .
$$

It holds for a large regime of values as visible in Figure 5.6 as long as the network is uncollapsed. Lower temperatures than the ones shown are expected to show the same behaviour.

\subsubsection{Effect of external tension}

The situation changes when the system is subject to external tension $\tau$. It is either pulled, which means $\tau>0$, or squeezed, which means $\tau<0$. 
Figure 5.7 shows the development of the area for positive and negative tensions.

Squeezing the system leads to an earlier collapse of the system. The exact value $\tau$ where the collapse happens depends on the temperature. The lower the temperature, the more external pressure a network can sustain.

However, if the temperature is low, the area development is dominated purely by external pressure for much lower values of $\tau$. Supporting tension prevents collapse for the temperatures used here and leads to lower thermal expansion. Above a temperature-dependent value of $\tau_{s}(T)$ the area of the system is extended beyond the area at zero temperature, i.e. $A>A_{0}[74]$. The higher the temperature, the longer the system sustains the contraction against pulling external tension. Above $\tau_{s}(T)$ the area is completely dominated by the tension and linearly extended with $\tau$. 

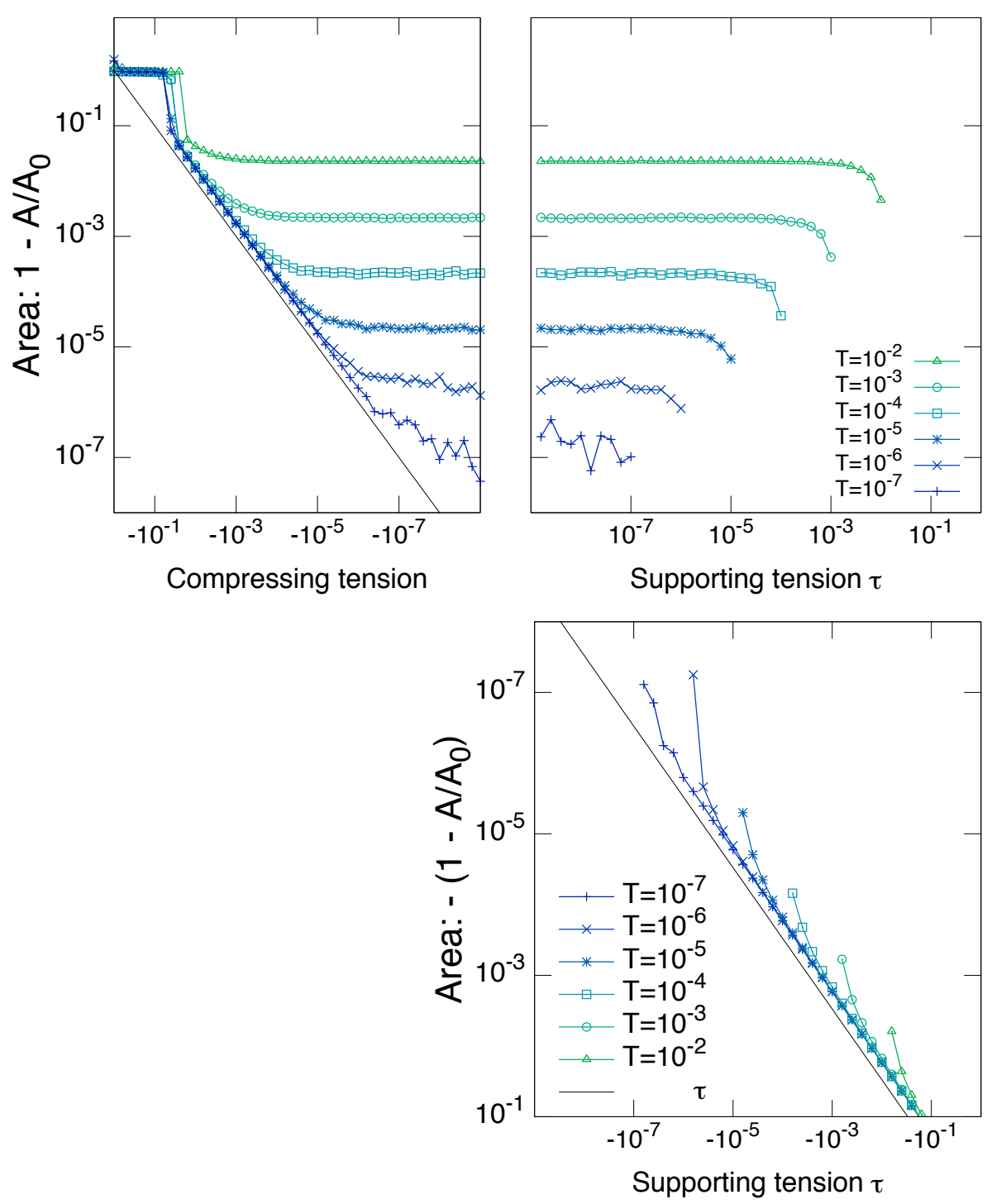

Figure 5.7: Fixed pressure Monte Carlo simulation of a spring network with coordination $z=4.9495$ in dependence of external tension $\tau$. Compressing tension leads to collapse of the system if it is larger than a certain value depending on the temperature of the system. Supporting tension leads to a total area $A>A_{0}$ for a certain temperature-dependent value of $\tau$. The black line marks where the behaviour of the spring networks is dominated by $\tau$ and becomes independent from temperature $T$. In between those limiting cases the networks show negative thermal expansion. Above this value the area is dominated by the tension. 


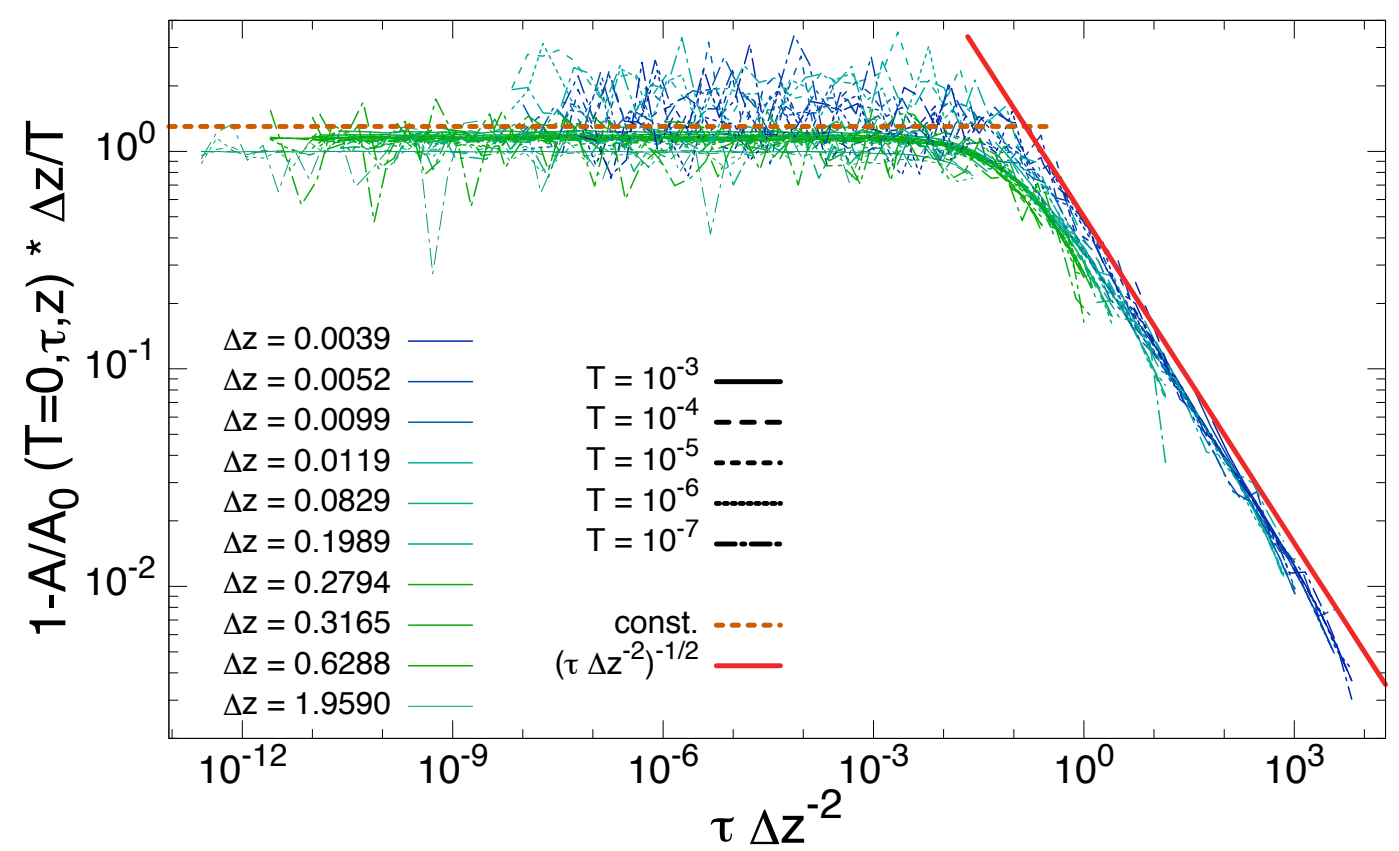

Figure 5.8: Scaling of system's area at fixed pressure for temperatures $T$, coordination numbers $z$ and external tensions $\tau$. The $y_{-}$ axis is the negative thermal expansion coefficient (NTE) times the distance from the jamming point $\Delta z$. The predictions derived from our scaling arguments are plotted on top of the data. The dotted orange line indicates the predicted area development for vanishing tension. The solid red line indicates the predicted area development for dominating tension. The data is in decent support of the predicted scaling for five orders of magnitude in $T$ and approximately three orders for $\Delta z$. System size is $N=3200$.

\subsubsection{Scaling with temperature, coordination and tension}

The relative contraction is measured for different combinations of $T, z$ and $\tau$. The data is scaled on the $\mathrm{x}$-axis for $\tau \cdot \Delta z^{-2}$ and on the $\mathrm{y}$-axis for $N T E \cdot \Delta z$. In order to get close to the isostatic point and to be able to check the scaling for low $\Delta z$ the network size $N=3200$ is large in comparison to the data shown until now. The resulting scaling plot is shown in Figure 5.8.

For low coordination numbers the data is less accurate, see the blue curves in Figure 5.8. The reason is that simulating the system for low temperatures and low coordination numbers demands more computational time than for high temperatures and high coordinations. But for low coordinations we cannot tune the 


\section{Thermally Activated Networks of Springs}

temperature too high before the collapse of the spring network happens. That is why in the figure the data for coordinations close to the isostatic point gets less sharp. This will be more problematic when we compute the bulk modulus from the data.

The shape of the scaling functions can be extracted from the scaled simulation data.

We can now verify the scalings derived from analysis of the soft mode spectrum [61] with the simulation data (see Chapter 1.3.3). The predictions derived from our scaling arguments are plotted on top of the data. The limiting cases for $\tau \Delta z^{-2}<<1$ and $\tau \Delta z^{-2}>>1$ derived from the theory are decently supported by the simulations: at values of $\tau<<\Delta z^{2}$ the tension does not play a role, while $\tau>>\Delta z^{2}$ means that tension dominates the behaviour of the material. The data is in decent support of the predicted scaling for five orders of magnitude in temperature $T$ and approximately three orders of magnitude for the distance to isostaticity $\Delta z$.

Let us conclude with the side note that the scaling extends to negative tensions $\tau$ with a constant scaling function as long as the pressing tension is not too high. This prediction is supported by simulations, too. 


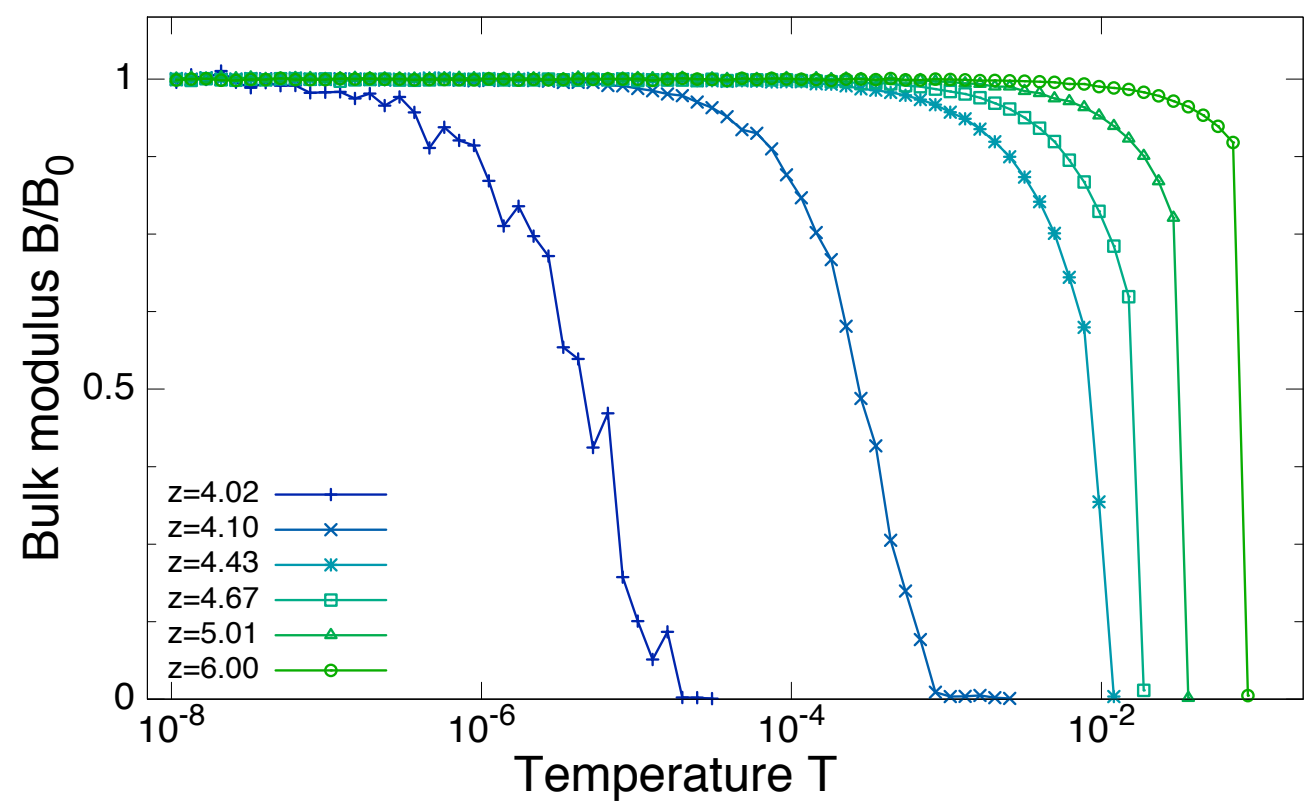

Figure 5.9: Bulk modulus in dependence of temperature $T$ for different $z$ at zero tension. The system softens with increasing temperature. The collapsed regime is not shown. System size $N=100 . N p T$ simulation.

\subsection{Bulk modulus}

Introducing temperature leads to contraction of the model system. It will surely also affect its other properties. Here we will investigate the bulk modulus $B$, which measures the material's resistance to uniform compression:

$$
B=-A \frac{d p}{d A}
$$

One could imagine that a decrease in area will lead to a hardening of the system and thus an increase of the bulk modulus. This is not the case.

\subsubsection{Dependence on temperature}

Figure 5.9 shows simulation data of the bulk modulus, defined in (4.18) in the $N p T$ ensemble at zero external tension. The system softens with increasing temperature even though the area is decreasing. 


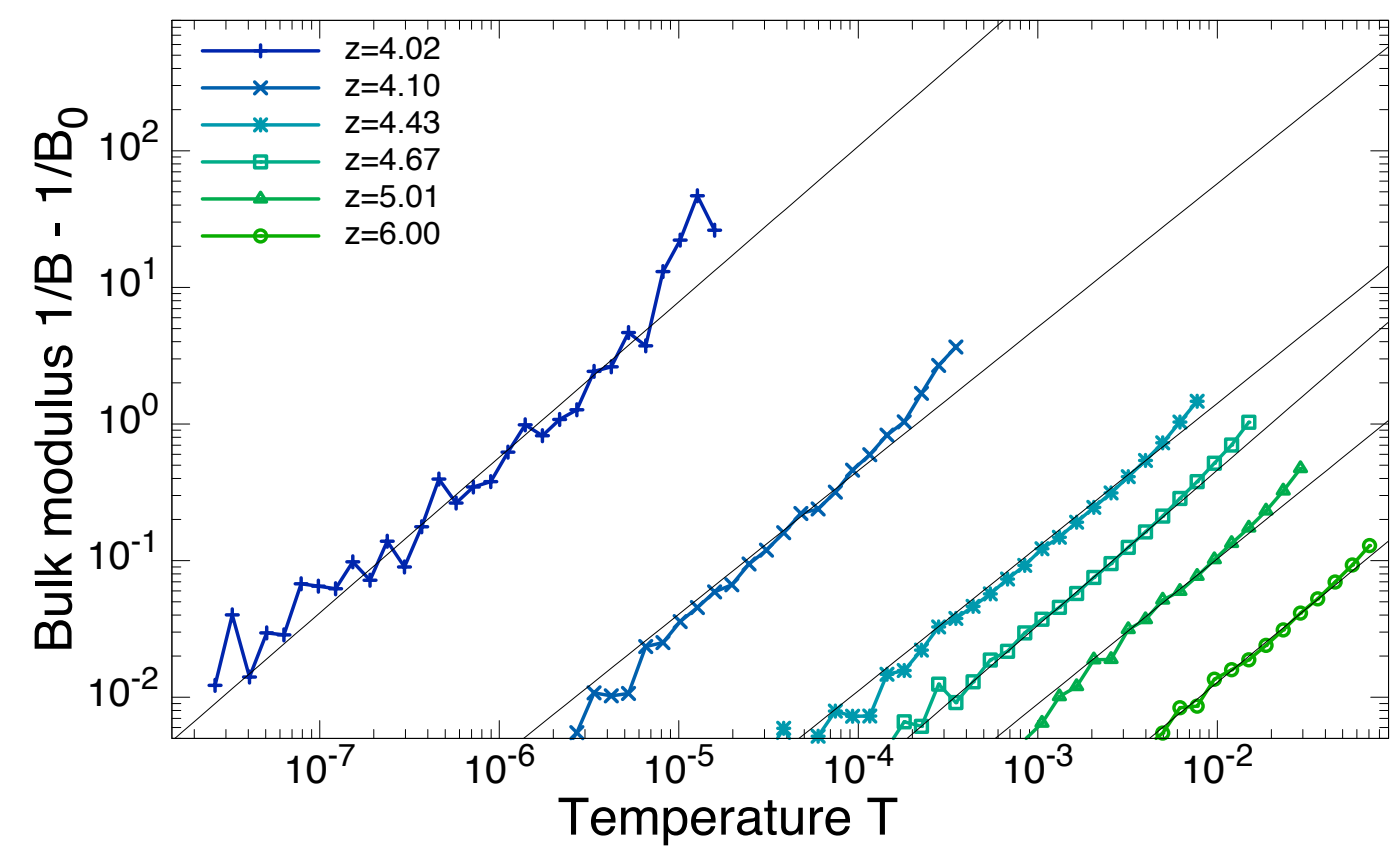

Figure 5.10: Inverse bulk modulus (compressibility) in dependence of temperature $T$ for different $z$ at zero tension compared to inverse zero-temperature bulk modulus. The system softens with increasing temperature. The black lines indicate the fitted function through a linear least squares fit of the logarithmized data. Exponents are found in the table in the main text. The dependence on temperature is linear. The collapsed regime is not shown. System size $N=100 . N p T$ simulation.

Figure 5.10 shows the inverse bulk modulus - which is the compressibility - as a function of $T$ compared to the inverse zero-temperature bulk modulus. We introduce this quantity because it is what we will use later for the scaling plot.

The data in Figure 5.10 can be used to determine the dependence on temperature. The black lines indicate the fitted function through a linear least squares fit of the logarithmized data. The following exponents are found for different coordinations of the spring network: 


\begin{tabular}{|l|l|}
\hline Coordination $\mathbf{z}$ & Exponent of T-dependence \\
\hline 4.021 & $1.13 \pm 0.06$ \\
\hline 4.1031 & $1.05 \pm 0.08$ \\
\hline 4.433 & $1.09 \pm 0.01$ \\
\hline 4.6735 & $1.13 \pm 0.02$ \\
\hline 5.0101 & $1.06 \pm 0.05$ \\
\hline 6.0 & $1.08 \pm 0.04$ \\
\hline
\end{tabular}

The limiting factor in determinig the exponents is the measurement of the bulk modulus at zero temperature. As the bulk modulus is measured through the fluctuations of the area of the system, we need another order of magnitude for the simulation time in order to get the same accuracy as for the area determination of the system. Higher values of $z$ yield more exact results as a larger set of measurements for large temperatures can be used because the system is still stable, i.e. not collapsed, for these temperatures.

Furthermore, the exponent is probably subject to finite size effects and thus depending on the relatively small system size as well.

From the exponents found in the table above we conclude that the dependence on temperature is approximately linear.

\subsubsection{Dependence on coordination}

In Figure 5.11 the bulk modulus is shown as a function of coordination for different temperatures. Following intuition the bulk modulus should stiffen with increasing connectivity.

The dependence for very low temperatures is similar to the athermal case. In the athermal case the bulk modulus decreases with connectivity and drops to zero below the isostatic point, which is defined by ill-connectedness and resulting mechanical instability.

Figure 5.12 shows schematically what is found in the simulations: despite the contraction - counter-intuitively - the material softens with increasing temperature. For $\Delta z \rightarrow 0$ the difference between temperatures diverges. At zero temperature the bulk modulus is vanishing for coordinations below the isostatic point $\Delta z<0$, and finite and increasing with $z$ for $\Delta z>0$. This discontinuous phase transition gets blurred at finite temperatures resulting in a sigmoid curve.

We are interested in the dependence of the bulk modulus on the coordination of the system. It should be clear that again the distance from the isostatic point will determine the scaling of the bulk modulus. 


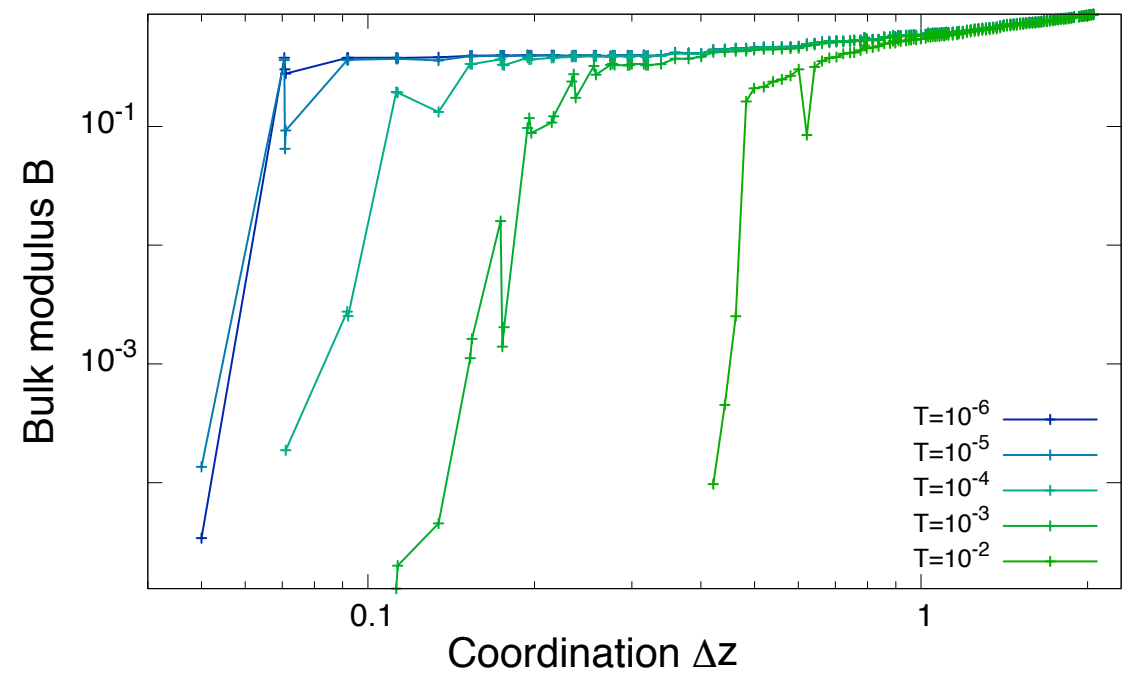

Figure 5.11: Bulk modulus in dependence of distance in coordination from the isostatic point $\Delta z$ for different temperatures $T$ at zero tension. The system softens with decreasing connectivity as expected. The collapsed regime is not shown. System size $N=100$. $N p T$ simulation.

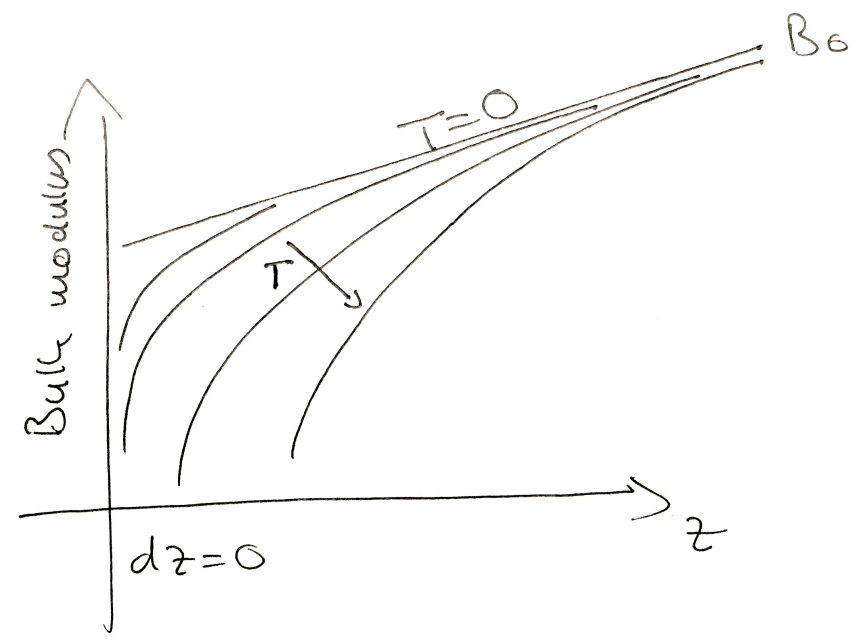

Figure 5.12: Bulk modulus of spring network as a function of $\Delta z$ for different temperatures. Schematic. For vanishing temperature the bulk modulus stays finite until the isostatic point. $B$ gets more and more softened with increasing temperature towards a sigmoidal curve. 


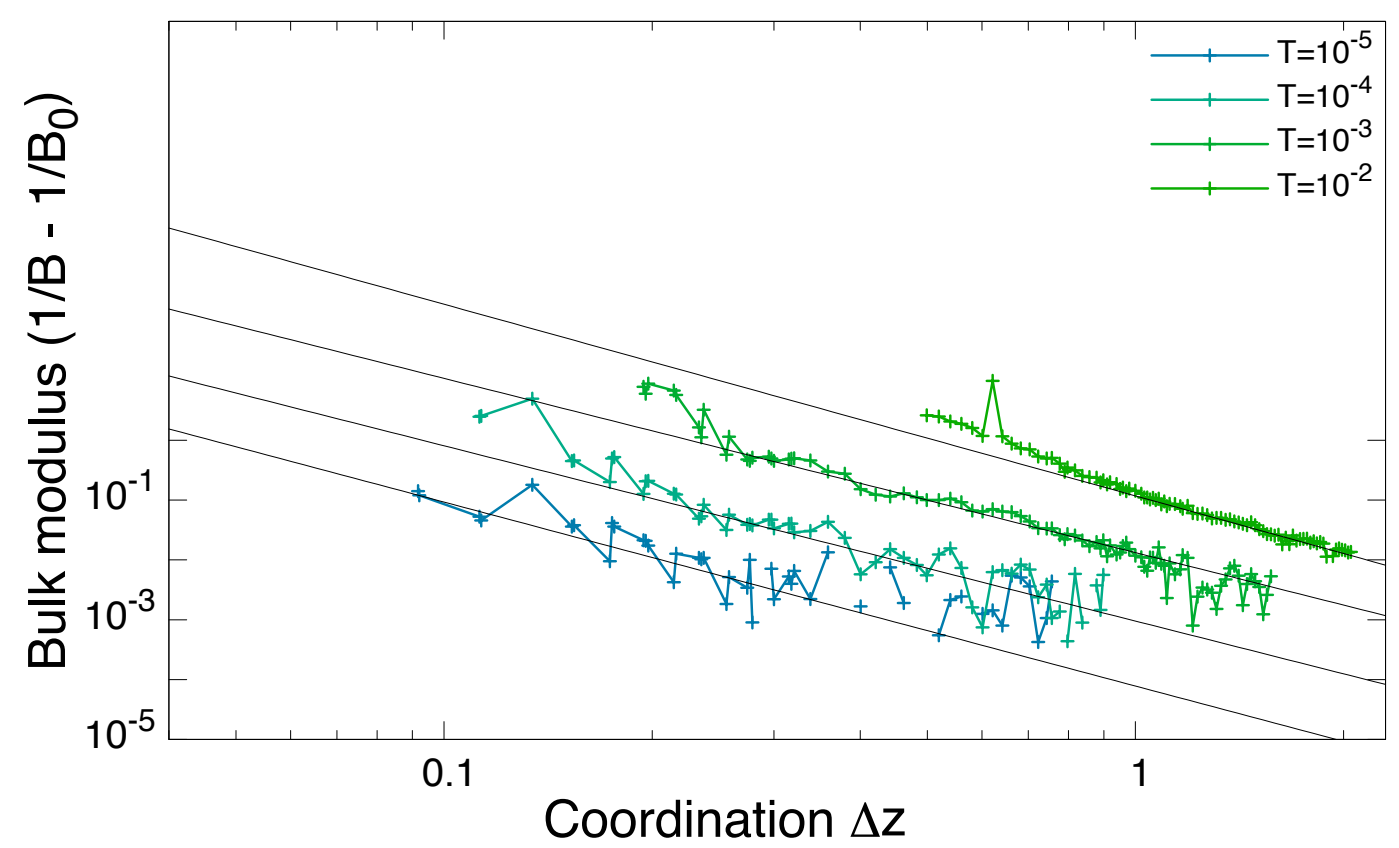

Figure 5.13: Compressibility compared to the athermal compressibility as a function of the distance from the isostatic point. Black lines indicate the fitted power functions for different temperatures. Exponents can be found in the table in the text. The relative compressibility scales with $\Delta z^{-3}$.

Figure 5.13 shows the compressibility compared to the athermal case as a function of $\Delta z$ for different temperatures.

The black lines indicate the fitted function through a linear least squares fit of the logarithmized data. The following exponents are found for different temperatures of the spring network:

\begin{tabular}{|l|l|}
\hline Temperature & Exponent of $\Delta z$-dependence \\
\hline $10^{-2}$ & $-3.20 \pm 0.17$ \\
\hline $10^{-3}$ & $-2.91 \pm 0.15$ \\
\hline $10^{-4}$ & $-2.93 \pm 0.24$ \\
\hline $10^{-5}$ & $-3.07 \pm 0.53$ \\
\hline
\end{tabular}

Again for low temperatures the data is less accurate, yet the results indicate a dependence of the compressibility where the coordination scales like $\Delta z^{-3}$.

Hence, for the zero-tension case we get: 


$$
\frac{1}{B}-\frac{1}{B_{0}} \sim \frac{T}{\Delta z^{3}}
$$

\subsubsection{Scaling with temperature, coordination and tension}

The bulk modulus is measured for different combinations of $T, z$ and $\tau$.

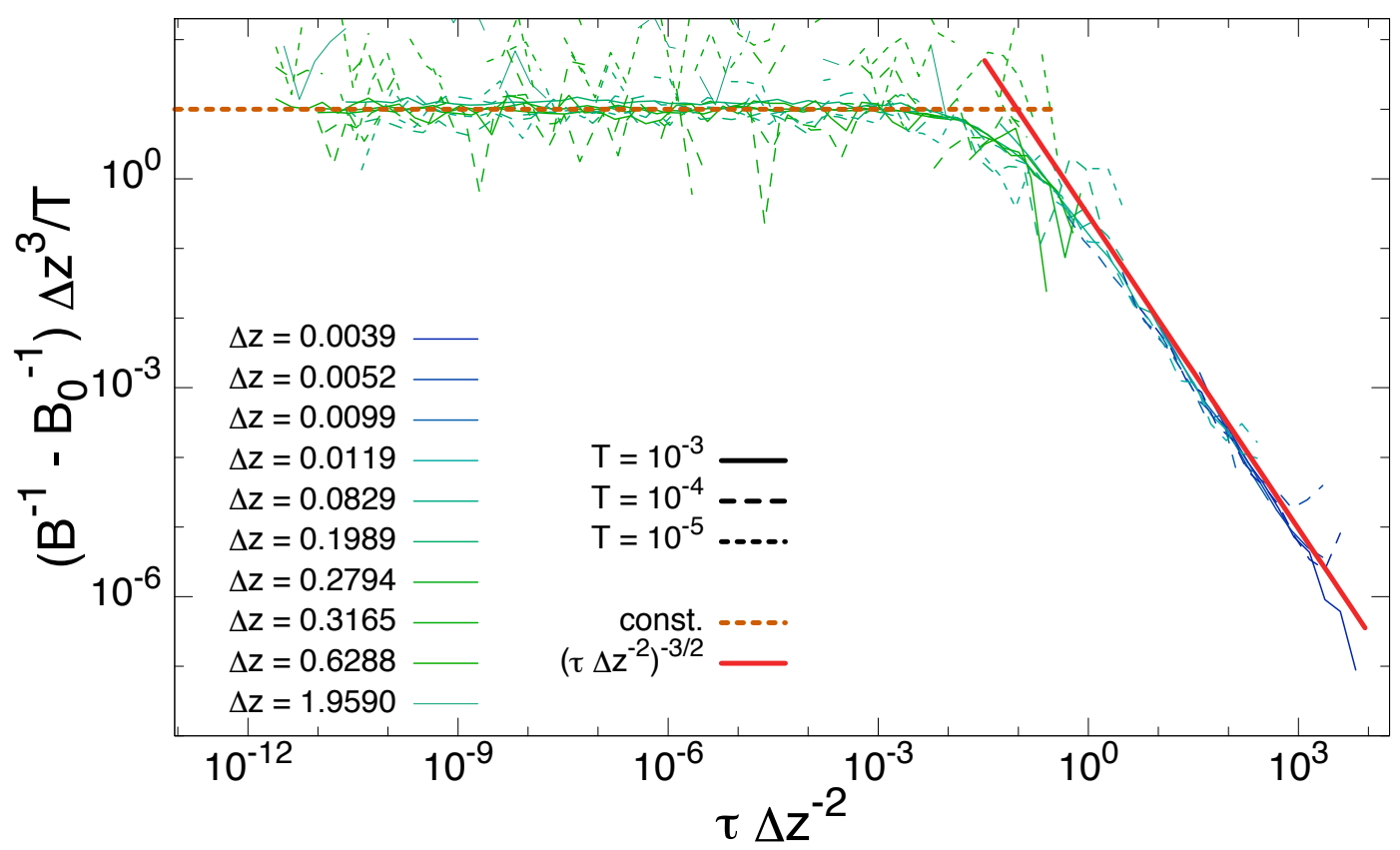

Figure 5.14: Rescaled data for the (inverse) bulk modulus. The dotted orange line indicates the predicted inverse bulk modulus for vanishing tension. The solid red line indicates the predicted inverse bulk modulus for domination tension. Predictions derived from scaling arguments are plotted on top of the data. The data is in decent support of the predicted scaling for three orders of magnitude in $T$ and $\Delta z>0.01$. Smaller $\Delta z$ support the scaling only for $T=10^{-3}$ and $T=10^{-4}$ and dominating tension. System size is $N=3200$.

If we want to compute the bulk modulus the fluctuations of the area are needed. Hence, in order to get data of the bulk modulus that are equally sharp as those of the area we would even need another order of magnitude of more simulations. This is why the agreement with the scaling prediciton is only good for larger temperatures $T$ in the range $10^{-3}-10^{-5}$. In this temperature regime, however, 
$\Delta z \lesssim 0.01$ collapses for vanishing tension. Still, the overall agreement with the scaling is strong.

The scalings derived from mode analysis following Chapter 1.3.3 are applied to the simulation data of the bulk modulus in Figure 5.14. The simulation supports the predicted scaling. 


\subsection{Shear modulus}

Let us consider the shear modulus, which measures the resistance of a material to deformations induced by stress which operates coplanar to the material's cross section. In our case this means that the angle $\alpha$ between the volume-enclosing vectors $U$ and $V$ is changing, see Figure 3.1. Except in the case of collapse, $\alpha$ will fluctuate around orthogonality. The shear modulus $G$ is defined as the proportionality factor connecting shear stress $\tau_{\text {shear }}$ and shear strain $\alpha_{\text {strain }}$ :

$$
\tau_{\text {shear }}=G \cdot \alpha_{\text {strain }}
$$

The angle $\alpha_{\text {strain }}$ is the material's response to the shearing force. For small deformations we can approximate this angle by the change of vector $V$ in the $x$ direction.

The force $\tau_{\text {shear }}$ is the shearing force trying to deform the system. In the Hamiltonian of the system (3.5) there is no apparent active shearing force. Indeed, this element is not included in the model. However, the Monte Carlo moves of $U$ and $V$ allow a change of the simulation box's shape, which introduces shear fluctuations. This change in shape is mapped so that $U$ always has a zero $y$-direction component. Consequently, the fluctuations in $V$ allow us to compute the shear modulus, see (4.19).

We are interested in what happens to the shear modulus of jammed spring networks at a constant volume when we excite their soft modes through temperature. The system's volume cannot contract because we keep its size fixed, but it is allowed to change its shape. At simulation time $t=0$ the simulation box is a square so the changes in shape will make the box a rhombus.

Now, at fixed volume the network cannot contract as it would at fixed pressure. This force cannot vanish. If we fixed the volume's shape, it would have to increase the system's pressure - this situation will be discussed in chapter 8. Allowing the volume to change we expect the force to increase the shear modulus, too. This is commonly referred to as shear stiffening.

Analysis of soft modes gives [61] a predicition.

In Figure 5.15 we plot the results for $G$ of $N V T$-simulations for different temperatures and coordination numbers together with the scaling obtained from considerations on the mode spectrum.

Even for very high temperatures where $T$ is of the order of the spring constant $k$ the scaling holds well. Shear stiffening is an interesting phenomenon as such. 


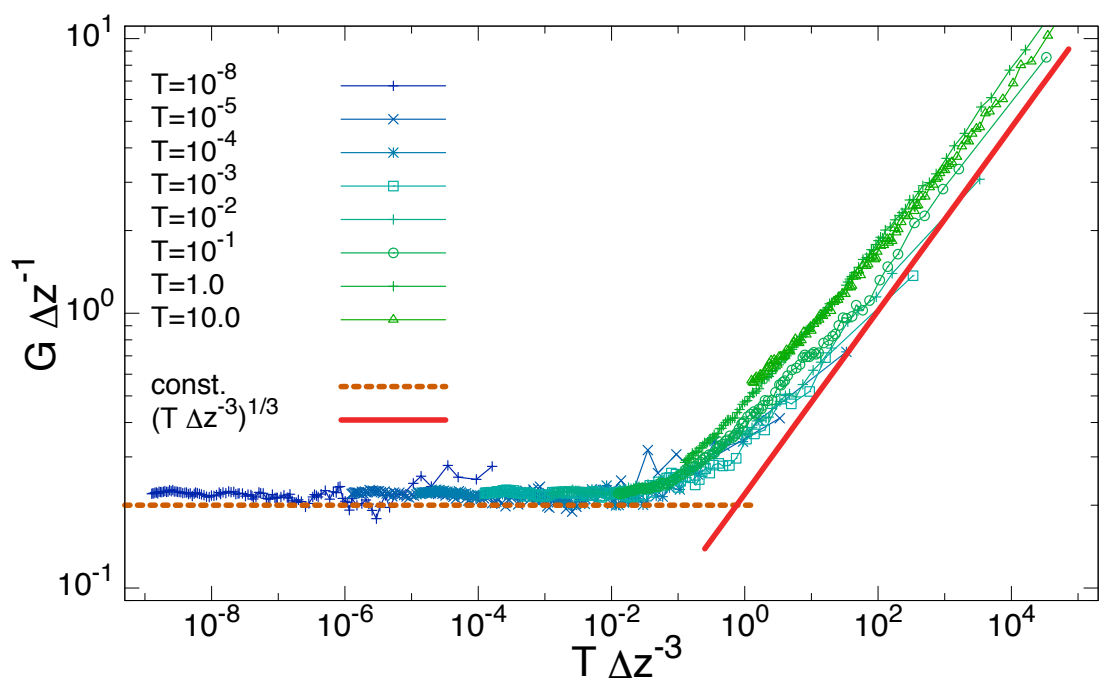

Figure 5.15: Scaling of shear modulus in the canonical ensemble at deeply spaced coordinations and several temperatures. The scaling derived from the soft mode analysis is strongly supported by the simulation data. The data is for eight orders of magnitude in temperature and deep in $d z$ from $0.01-2.0$. $10^{10}$ Iterations per $(z, T)$-configuration including equilibration. System size $N=1600$.

Metals, for instance, usually have a shear modulus decreasing with temperature. In the case of our system it may be realized that $G$ can be tuned quite sensitively through $\Delta z$ or - in other words - the number of contacts. The scaling of the shear modulus is another support for our theory. 


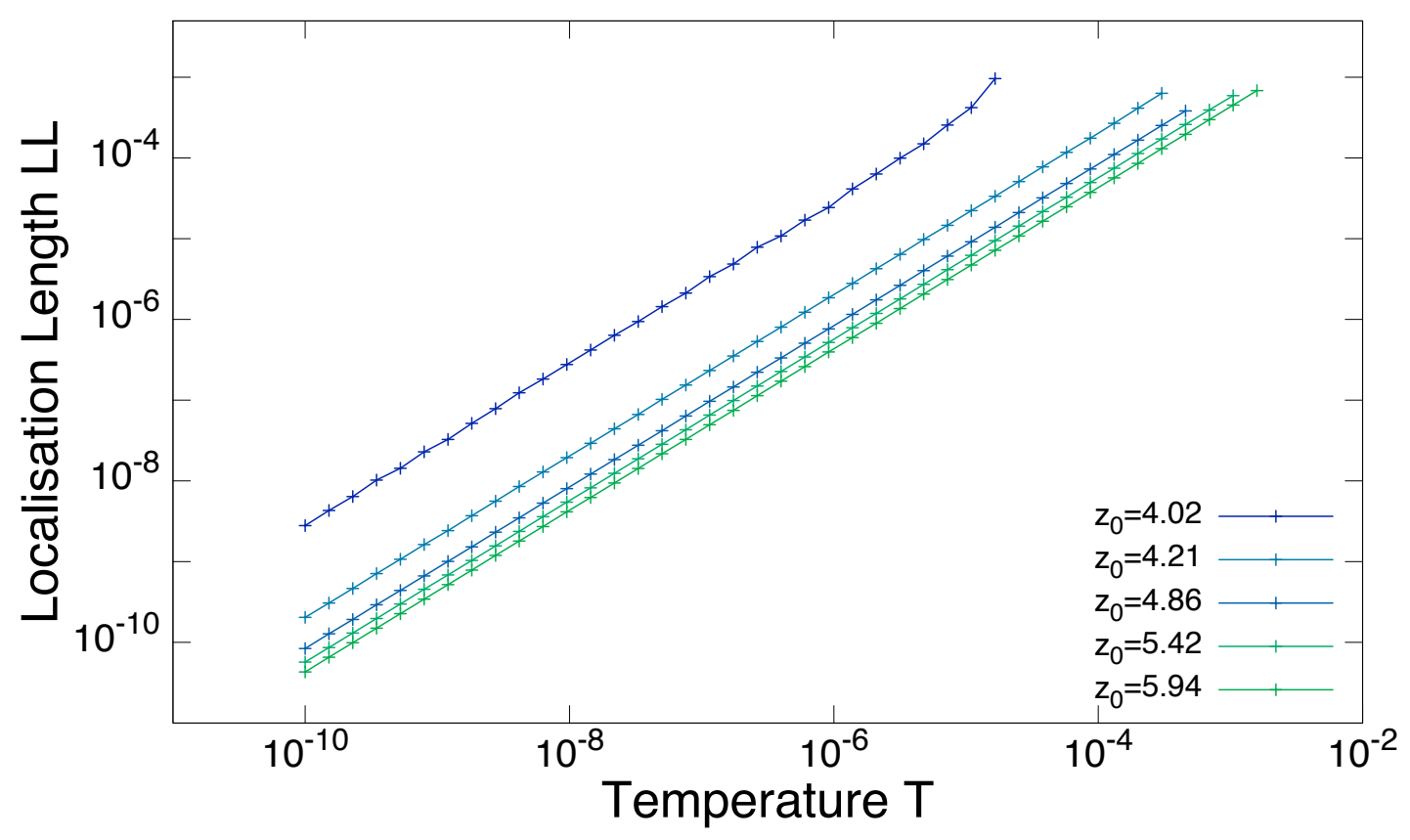

Figure 5.16: Localisation length as a function of temperature $T . N=100$, $\tau=0.0$

\subsection{Localisation length}

As a dynamic - in contrast to thermodynamic - quantity we determine the localisation length of vertices in spring networks derived from jammed packings. The localisation length $L L$ is the average variance in displacement of vertices in the system, defined as in (4.20) and measured through the algorithm defined in Section 4.3.9.

By intuition it should increase with temperature as thermal fluctuations will lead to greater exploration of the space surrounding a vertex. Furthermore, it should decrease with connectivity because a more connected vertex is more strongly constrained in its movement. Both intuitions are confirmed by simulation results and supported by theory.

Figure 5.16 shows the localisation length as a function of temperature for different coordinations.

The exponent for the temperature dependence is 1 for the whole range of coordinations: 


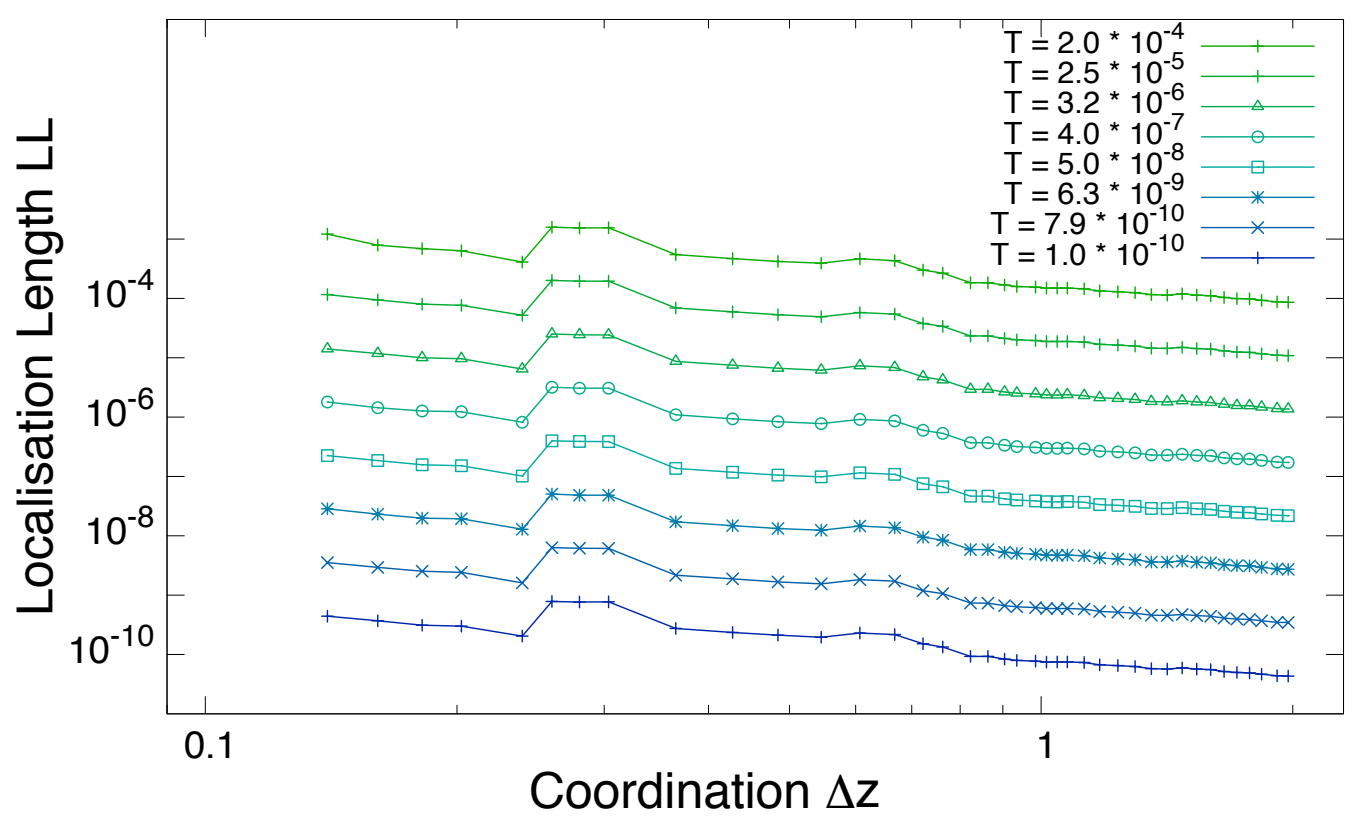

Figure 5.17: Localisation length as a function of $\Delta z . N=100, \tau=0.0$

\begin{tabular}{ll}
\hline Coordination $\mathbf{z}$ & Exponent of T-dependence \\
\hline 4.021 & $1.021 \pm 0.006$ \\
\hline 4.206 & $1.001 \pm 0.0003$ \\
\hline 4.860 & $0.99994 \pm 0.00008$ \\
\hline 5.420 & $1.00001 \pm 0.00006$ \\
\hline 5.940 & $1.00002 \pm 0.00006$ \\
\hline
\end{tabular}

Figure 5.17 shows the localisation length as a function of temperature for different coordinations.

The scaling with respect to $\Delta z$ is not as perfect as the temperature-dependence:

\begin{tabular}{ll}
\hline Temperature $\mathbf{T}$ & Exponent of $\Delta z$-dependence \\
\hline $1.0 \cdot 10^{-10}$ & $-1.18 \pm 0.09$ \\
\hline $7.9 \cdot 10^{-10}$ & $-1.12 \pm 0.08$ \\
\hline $6.3 \cdot 10^{-09}$ & $-1.06 \pm 0.07$ \\
\hline $5.0 \cdot 10^{-08}$ & $-1.03 \pm 0.06$ \\
\hline $4.0 \cdot 10^{-07}$ & $-1.14 \pm 0.08$ \\
\hline $3.2 \cdot 10^{-06}$ & $-1.12 \pm 0.08$ \\
\hline
\end{tabular}

Still, assuming $\Delta z^{-1}$, as the mode analysis suggests seems justifiable. Together with finite size effects, the dependence on coordination is strongly influenced by 


\section{Thermally Activated Networks of Springs}

the exact preparation of the packing from which we derive the spring network. For the relatively small networks we used here, there seems to be a rearrangement which results in the bump in the $\Delta z$ dependence.

We conclude that the localisation length is proportional to temperature $\mathrm{T}$ and inverse proportional to $d z$ :

$$
L L \propto \frac{T}{d z}
$$

This can not come as a great surprise because displacement of a vertex and the contraction of the whole volume are intimately connected and we already found the same scaling relation for the area of the system, see Chapter 5.1.

\subsubsection{Scaling with temperature, coordination and tension}

We will now investigate the dependence of the localisation length $L L$ on temperature $T$, coordination distance from the isostatic point $\Delta z$ and tension $\tau$, see Figure 5.18.

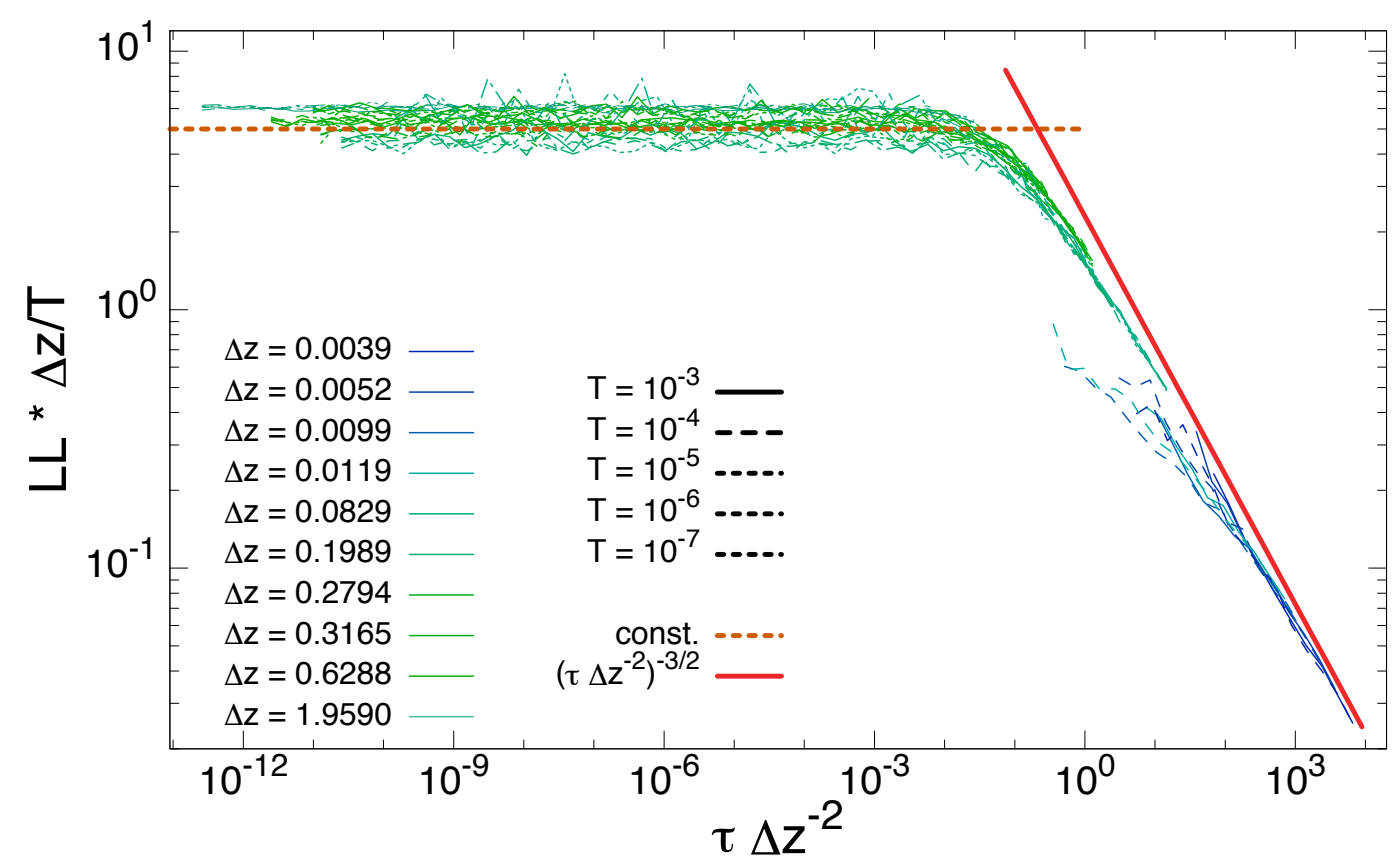

Figure 5.18: Scaling of Localisation length $L L$ times $\Delta z$ over T with $\tau$. $\Delta z^{-2}$. Localisation length (times by $\Delta z$ ) as a function of T. System size is $N=3200$. 
We can apply the scalings described in Chapter 1.3.3 to simulation data for $L L$. The support is strong in the case of $\Delta z \gtrsim 0.01$ but for a much larger range of temperatures compared to the results for the bulk modulus. Again, we have to measure fluctuations, i.e. the variance in distance, to get the localisation length. This is why low temperatures are problematic due to longer equilibration and correlation times. It is not entirely clear why low temperatures are still better represented than in the case of bulk modulus measurements. One vector to explain this might be the fact that in the case of the bulk modulus area fluctuations are needed and in the case of the localisation length $L L$ the variance in distance is considered. However, a vertex move happens as often as an area move in our simulation, i.e. at every $N^{\text {th }}$ steps.

Still, the results for coordinations $\Delta z \gtrsim 0.01$ decently support the predictions. 



\section{Dependence on network structure}

In this chapter we will test the effect of a network's structure on its mechanical properties. After all there are many to infinite ways imaginable to create a network with a certain average coordination number $z$. In our case we demand that the networks are only locally connected, i.e. only neighbours but no crossing links are allowed [83]. We consider the thermal expansion and the bulk modulus and how they are affected by temperature and coordination. The considerations shown here are mainly qualitative so that we can characterise jammed packing networks and emphasize their significance.

As networks to be compared with derived jammed spring networks we consider the following:

- Pruned networks obtained from jammed packings, starting with the pruning at different coordinations $\Delta z$.

- C4 networks: each vertex is connected to its 4 next neighbours while vertices at $T=0$ are distributed like the crossings on a chess board. Figure 6.1, left panel shows such a network being thermally activated.

- C6 networks: each vertex is connected to its 6 next neighbours while the structure is triangulated (with equilateral triangles at $T=0$ ).

- Pruned C6 networks: we start from a regular C6 network and take out links according to the described pruning protocol (see Chapter 2.3).

In Figure 6.2 the development of the relative decrease in area $1-A / A_{0}$ and the bulk modulus $B$ are shown for C4, C6 and pruned C6 networks in the top row and for pruned networks in the lower row. Both are compared to networks obtained from jammed packings.

C4 networks (Figure 6.2 top, pink line), C6 and pruned C6 networks (Figure 6.2 top, dotted lines) show negative thermal expansion over a large regime of temperatures. Traces of this behaviour could already be found in [66] and [84].

Also the pruned networks contract with temperature. We may conclude that this 

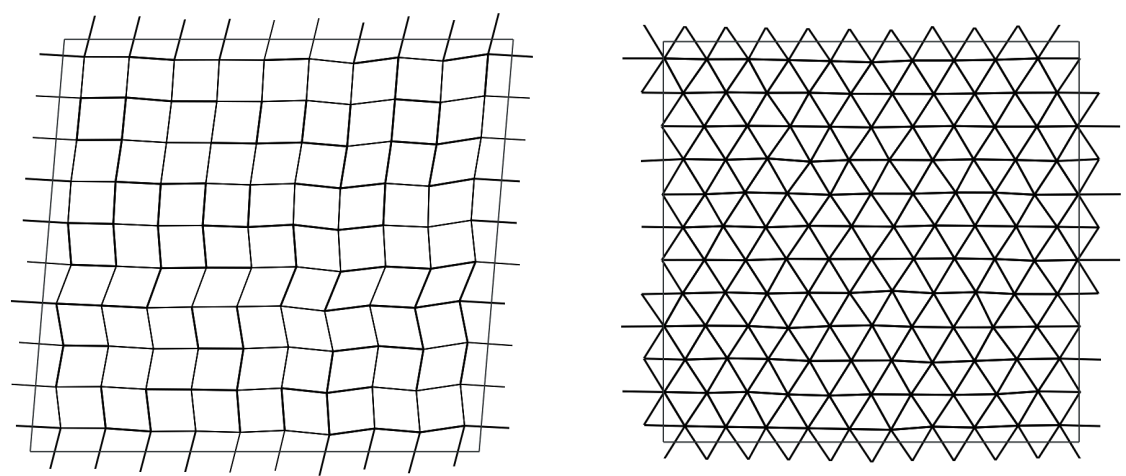

Figure 6.1: Structure of fourfold (C4, left picture) and triangulated (C6, right picture) networks. Both networks are shown thermally activated. In the network with fourfold connectivity we can see how temperature leads to soft deformations of the zero-temperature square structure of the network. Parallelograms and other shapes - favoured by entropy - form and make the whole system's area decrease [66].
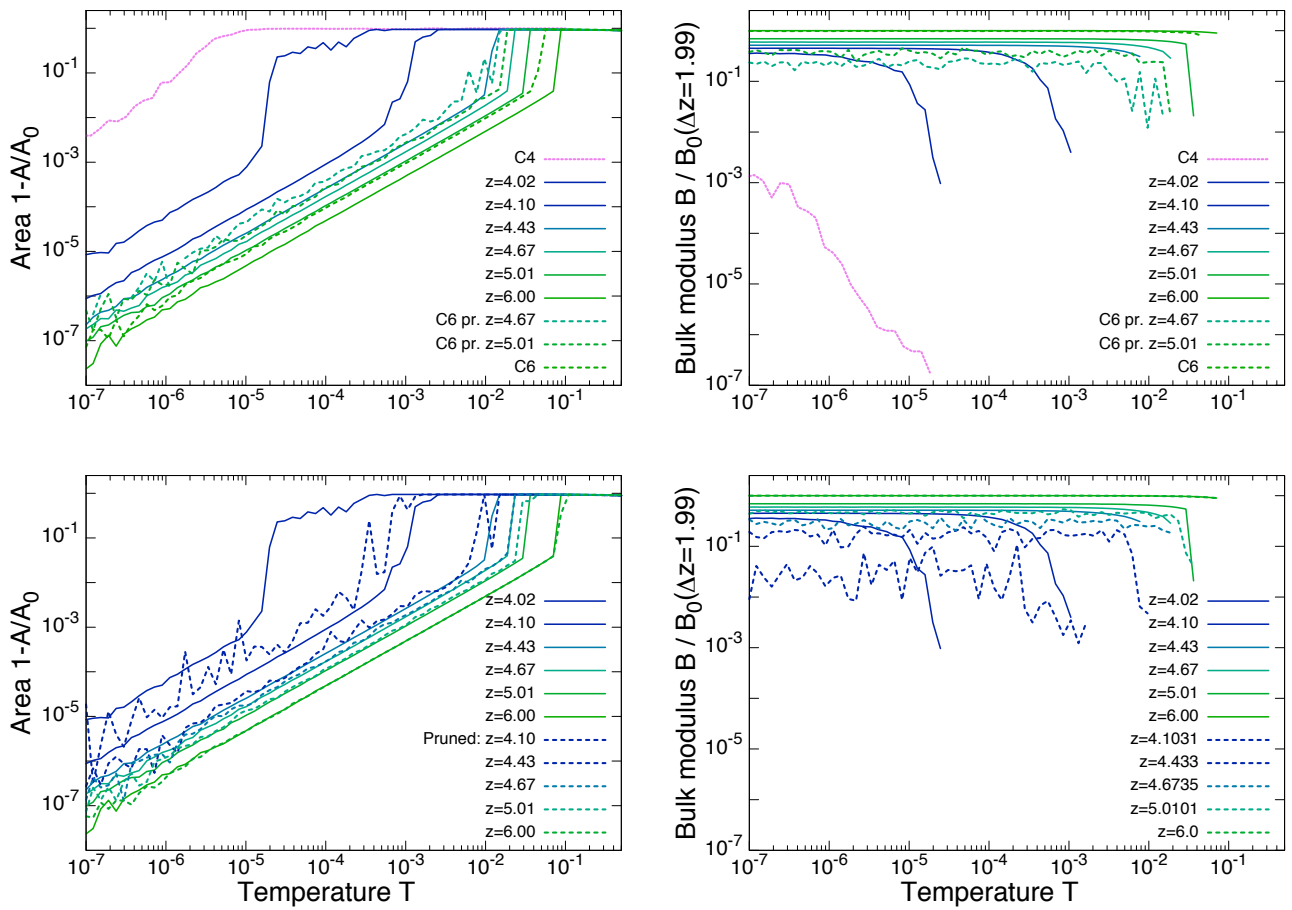

Figure 6.2: Relative contraction of area and bulk modulus for C4, C6 and pruned C6 networks (top figures) and for pruned jammed packing spring networks (bottom) compared to networks from jammed packings. 
is a general property of spring networks at vanishing tension and that they do so directly proportionally to temperature $T$.

To which extent this is the case at finite positive and negative tensions needs to be explored further. C4 networks according to [66] show NTE only for "some" positive tensions and expand for all negative tensions $\tau<0.0$. If this is only due to the limited range of tensions in the study needs to be further clarified. In any case we showed in Figure 5.7 and the scaling of the area, Figure 5.8, that our jammed packing networks maintain the NTE property for $\Delta z^{-2} \tau<<1$ while it vanishes quickly when the tension begins to dominate for $\Delta z^{-2} \tau>>1$.

We will now consider the onset of collapse and the bulk modulus. C4 networks seem to show no transition into the fully contracted state where $1-A / A_{0} \rightarrow 1.0$. They reach this state for lower temperatures than jammed packings with the same number of links. C6 and pruned C6 networks reach the collapse earlier as their counterparts derived from jammed packings with the same number of links and were found to be unstable for coordinations below a certain threshold around $\Delta z \approx 0.5$. Furthermore, C4 networks and pruned C6 networks were found to be much softer when considering the bulk modulus. C6 networks seem to be of an order similar to fully connected jammed packings.

Pruned networks show only a slightly earlier collapse with T. More importantly their bulk modulus behaves very differently from original jammed packings. This stems from cutting links this way leads to rigidity percolation $[15,85,86]$.

Figure 6.3 shows the bulk modulus as a function of $\Delta z$ for jammed packing networks and their pruned counterparts while we started pruning at two different values of $z$.

We see that the bulk modulus of the pruned networks vanishes quickly with $\Delta z$ from the point on where pruning starts. The scaling with coordination is roughly $B \propto \Delta z^{1.5}$ known from [64] when approaching isostaticity. The networks for jammed packings maintain a finite bulk modulus in the athermal case [2] and for low temperatures. In contrast to the slow divergence of the pruned networks close to isostaticity they diverge abruptly close to $\Delta z=0$. Approaching the isostatic point they show the characteristic softening observerd alread in Chapter Figure 5.11.

In the pruning algorithm there is an element of random choice of links to be removed. This results in different pruned networks for different runs of the algorithm even though the network we started with was the same. We implicitly check the variance induced by pruning in such a way that each $\Delta z$ seen in Figure 6.3 stems from an individually pruned network. This is why the data becomes less sharp (with increasing $\Delta z$ ). For the purposes of the considerations here the variability induced by pruning is not an important factor. 


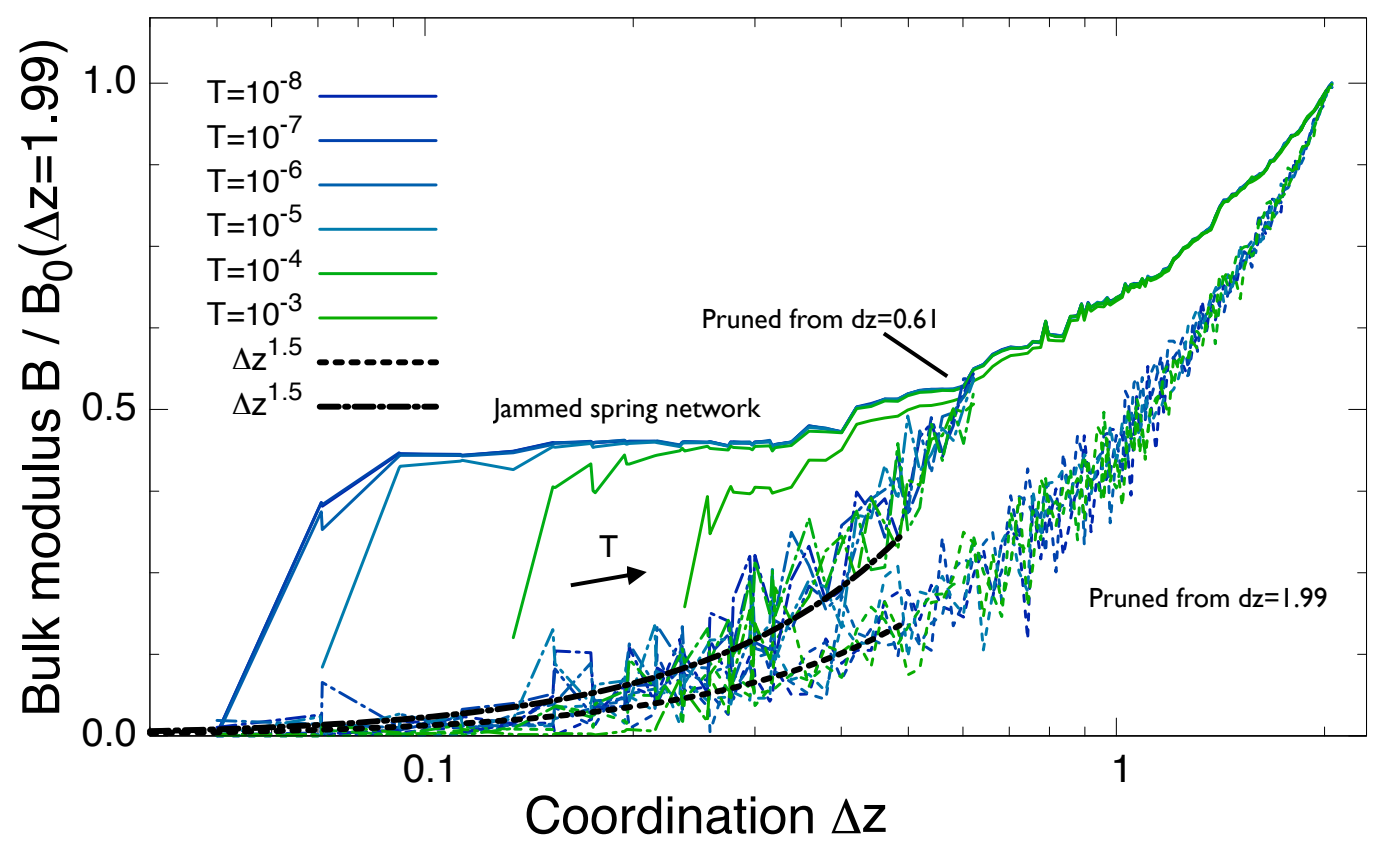

Figure 6.3: Bulk modulus for networks from packings (solid line) and their pruned counterparts where pruning started from $\Delta z=$ 1.99 (dashed line) and $\Delta z=0.61$ (solid and dotted line). Data as a function of the distance from the isostatic point of the jammed packing spring networks. The bulk modulus is compared to its value at zero-temperature far away from the isostatic point $\Delta z=1.99$. Strongly vanishing bulk modulus with $\Delta z$ from where pruning starts for both pruned networks with scaling $\propto \Delta z^{1.5}$ [64] approaching isostaticity. The networks for jammed packings maintain their bulk modulus approaching the isostatic point unless they get softened by temperature. In the limit of zero temperature the networks obtained from jammed packings have a large bulk modulus while the one of pruned networks vanishes, also see [2]. The wiggly data for pruned networks stems from every $\Delta z$ data point being pruned independently. Hence, on a qualitative scale we can say that the random component which induces variability in the pruning algorithm plays no important role. System size is $N=100$. 


\section{Phase diagram of jammed spring networks}

In this chapter we will present the phase diagram of networks of jammed sphere packings. Until now the behaviour of networks of a given coordination $\Delta z$ under tension $\tau$ and subject to temperature $T$ has been considered in a range where the structure and a finite volume of the system is maintained. When the temperature gets too high the system's volume collapses to almost zero. We determined the exact parameters where this transition happens.

The area at zero temperature $A_{0}$ to which we compare the current area $A$ to, is measured at a given $\tau$ of the system at $T=0.0$.

Figure 7.1 shows examples how the transition is approached with temperature for different coordinations $\Delta z$ and tensions $\tau$.

For low tensions at a given $\Delta z$ the transition is sharply defined with a large jump in area. With increasing $\tau$ the transition is shifted towards higher temperatures and the size of the jump $A_{j}$ gets smaller. For very large tensions, i.e. $\tau>1.0$, the system gets extended without boundariess.

We detect the position of the transition by finding the largest change in area with temperature for fixed values of $z$ and $\tau$. This is achieved by shifting a window of given size through the values and measuring the slope through linear regression. The algorithm records the temperature at which the transition happens and which area $A_{j}$ it had at the jump. The jump size at the transition would be $1-A_{j}$. We expect $A_{j}$ to decrease with increasing tension $\tau$.

Figure 7.2 shows the measured jump size as a function of $\tau$ for different $\Delta z$.

The top panel shows the jump size which we measured with the method described in the text. For a particular value of $\tau$ the data gets more noisy and it becomes next to impossible to estimate the jump size. This is where the system goes continuously from the contracted to the collapsed state of matter. We can think of it as analogue to the liquid-vapour transition with a critical point and the procedure to identify the position of the jump as a Maxwell construction [87]. 
7 Phase diagram of jammed spring networks
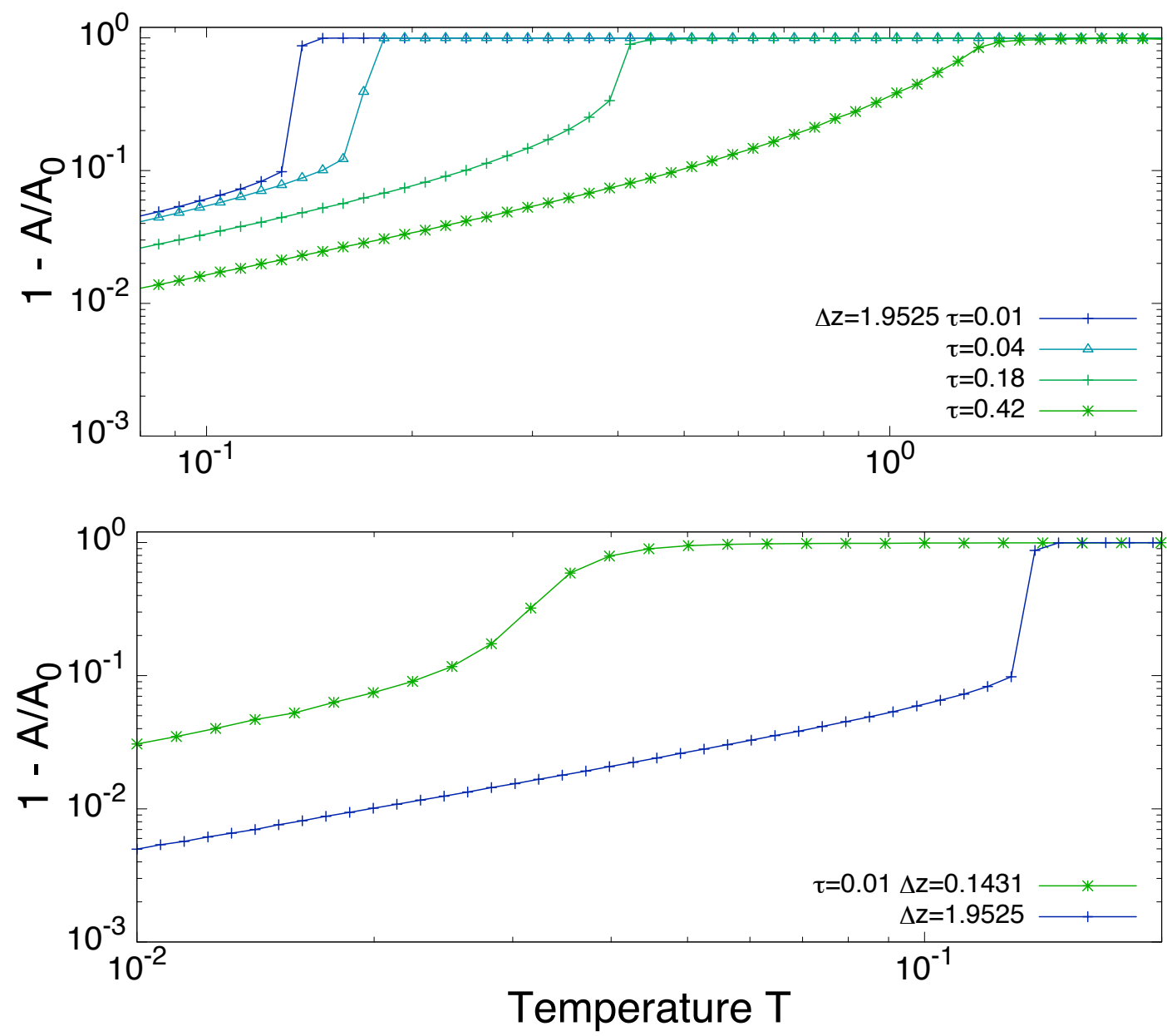

Figure 7.1: Both figures show the relative area contraction $1-A / A_{0}$ as a function of temperature for different tensions at constant coordination (top) and at constant tension for two different coordinations (bottom). Top: It can be seen that temperature $T_{c}$ at which the transition from the contracted to the collapsed shifts with increasing tension $\tau$. Furthermore, the jump measured in relative area becomes smaller and gets blurred in the case of $\tau=0.42$. Bottom: At a finite tension $\tau$ we can see that close to isostaticity(green) the transition is already quite blurred while it is sharp far away from isostaticity (blue). 


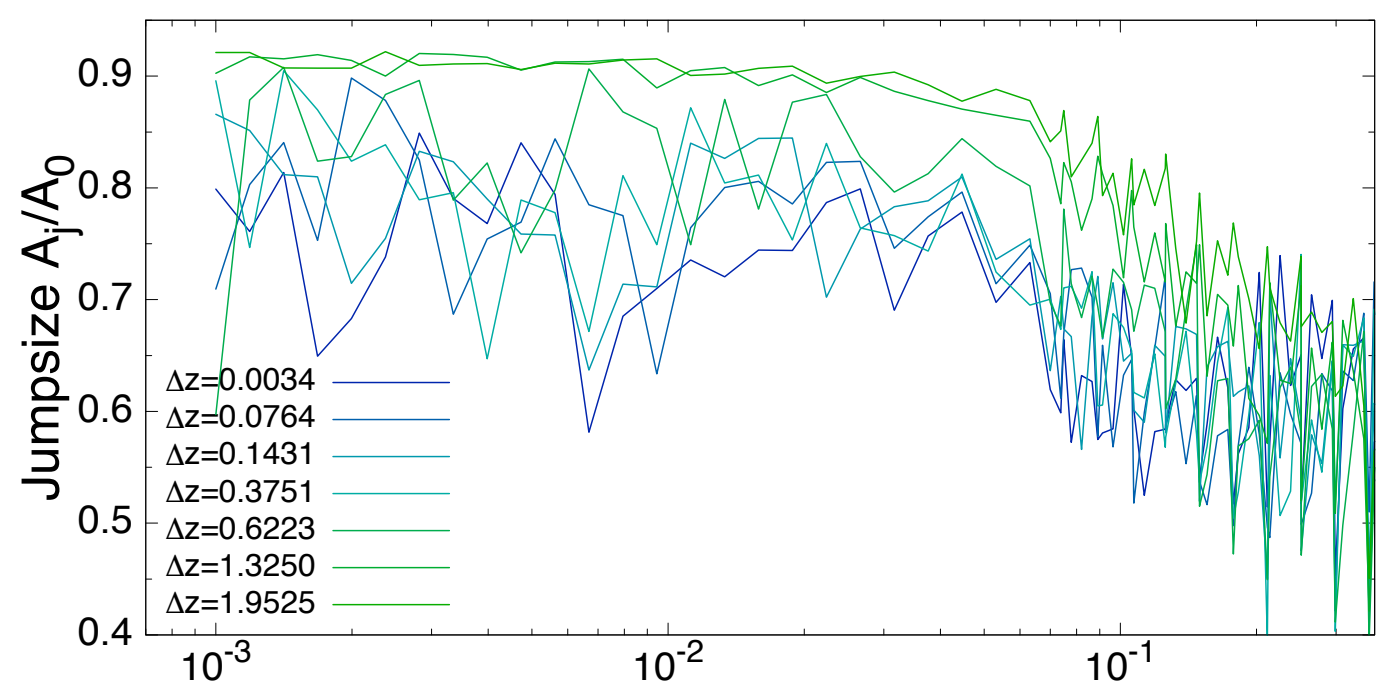

Figure 7.2: Area at jump $A_{j} / A_{0}$ at transition as a function of tension $\tau$ for different coordinations $\Delta z$. The top panel shows the jump size which we measured with the method described in the text. For a particular value of $\tau$ the data gets more noisey and it becomes next to impossible to estimate the jump size with the resolution given by the method. This is where we hypothesize that the system goes continuously from the contracted to the collapsed state of matter. This is strongly supported by visual inspection of the area development with temperature for $(\Delta z, \tau)$ around the critical point. 


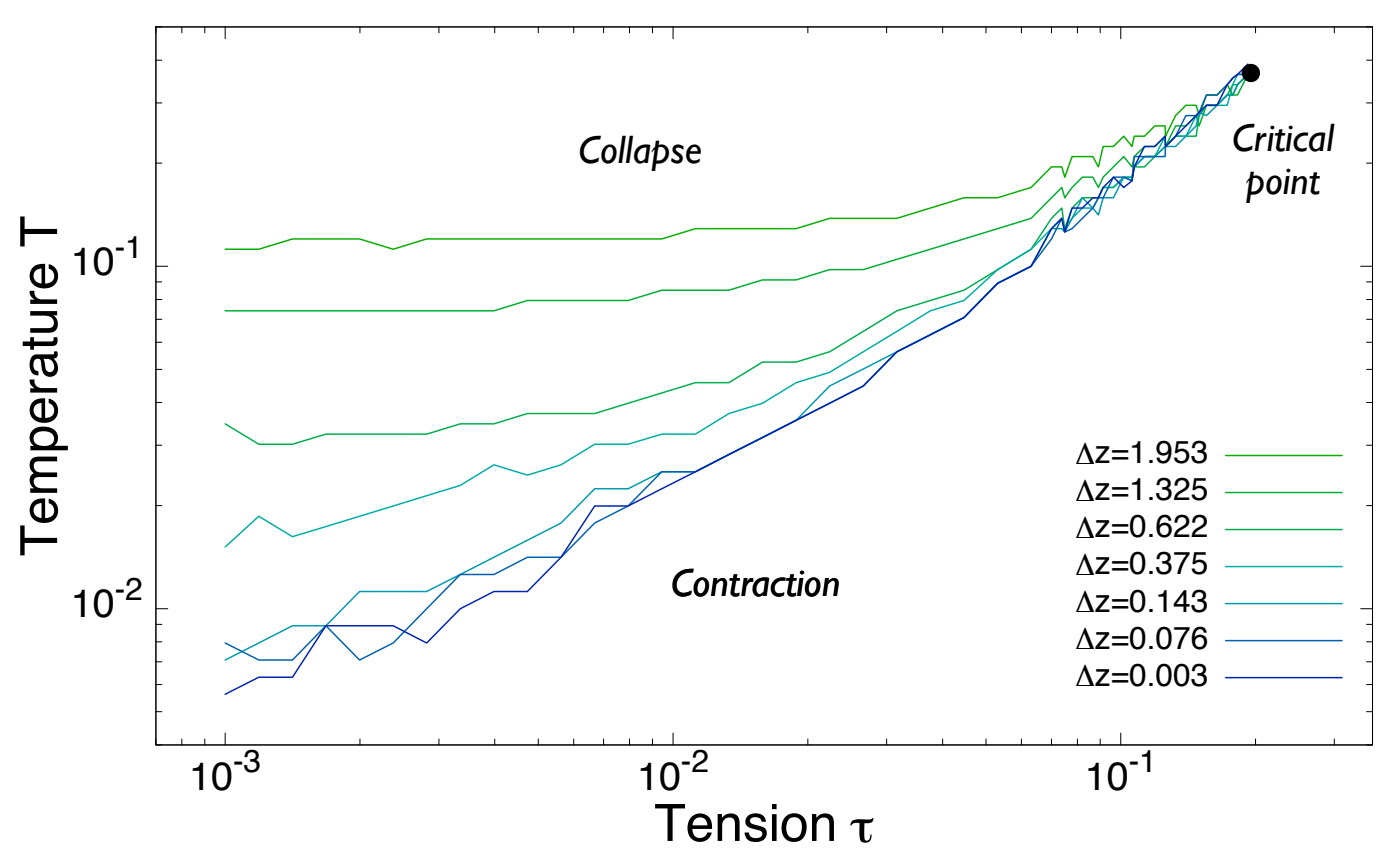

Figure 7.3: Phase diagram of spring networks obtained from jammed packings in the $T$ - $\tau$ plane. The system has two states: it either is contracted or collapsed. Lines mark the phase transition for different coordinations $\Delta z$. The system has a critical point where contraction changes into collapse continuously. Around the critical point the density of parameters was increased. Values for 67032 combinations of $(\Delta z, \tau, T)$ were used to compute the phase diagram. System size $N=3200$.

Figure 7.3 shows the phase diagram of spring networks obtained from jammed packings.

As pointed out the system has two states in which it is either contracted or collapsed, and the lines in the figure mark the phase transition for different coordinations $\Delta z$. We hypothesize a critical point where contraction goes into collapse continuously. This hypothesis is supported by visual inspection of the area development with temperature for $(\Delta z, \tau)$. Furthermore, this is a valid assumption taking into account the resolution of the method used. 


\section{Jammed Packings of Spheres}

In this chapter we will go back to jammed packings as such and investigate their response to temperature. In particular, we want to explore how jammed packings differ from spring networks, and on the other hand, what we can predict from the networks for the packings.

This study is conducted in the canonical ensemble $(N V T)$, i.e. we keep the area $A$ of the system fixed. Shear movements are excluded, too.

In the isothermal-isobaric ensemble - $N p T$ - we saw that spring networks contract with increasing temperature. In the $N V T$ ensemble this behaviour should show as a decrease in pressure. On the other hand, a dense packing cannot contract we might think. This is based on the fact that its pressure was already minimized given a certain packing fraction during the generation protocol.

One could expect that $z_{\text {eff }}$ grows with $T$ as each sphere in the packing explore more of the system and thus sees more links. This, however is not the case for low temperatures [88].

The Figure 8.1 shows how key parameters and quantities in packings and networks relate.

It is trivial but still important to remember that spring networks have a fixed connectivity, which means that the coordination is independent of temperature, i.e. $z(T)=z$. Packings have a constant packing fraction $\phi$ at fixed-volume. However, to be consistent with the earlier work on networks we will mainly use $z_{T 0}$ for packings, which is the coordination number at $T=0$. This quantity is directly comparable to spring networks and related to the packing fraction through the square root scaling $\Delta z_{T 0}^{2} \propto \Delta \phi$.

\subsection{Pressure development in $N V T$}

We want to know if the negative thermal expansion seen in spring networks transfers to jammed sphere packings. If this is the case we will see a decrease in pressure with temperature, analogue to a contraction of the system's volume in the $N p T$-ensemble. 


\begin{tabular}{|c|c|}
\hline Networks & Packings \\
\hline \hline Temperature $\mathrm{T}$ & Temperature $\mathrm{T}$ \\
\hline Volume $\mathrm{V}$ & Volume $\mathrm{V}$ \\
\hline Pressure $\mathrm{p}$ & Pressure $\mathrm{p}$ \\
\hline Coordination number $\mathrm{z}$ & Packing fraction, $T$-independent $\phi$ \\
\hline Coordination number $\mathrm{z}$ & Coordination number $z_{T 0}$ at $T=0$ \\
\hline Coordination number $\mathrm{z}$ & Effective coordination number $z_{e f f}(T)$ \\
\hline
\end{tabular}

Figure 8.1: Comparison of key quantities in networks and packings of spheres. The main difference is that networks by definition do not change their (topological) structure while packings generally do. Hence, the true constant for a packing is its packing fraction $\phi$. To be consistent with the earlier work on networks we will mainly use $z_{T 0}$ for packings, which is the coordination number at $T=0$. Furthermore, to quantify the change in structure we measure the effective coordination number $z_{e f f}(T)$, which is dependent on temperature.

We define the isochoric thermal pressure coefficient $\gamma$ :

$$
\gamma=\frac{1}{p}\left(\frac{\partial p}{\partial T}\right)_{V}
$$

To make packings and networks directly comparable, the spring networks will be loaded at $T=0$, i.e. the rest-length of a spring is set to its sum of radii of interacting spheres from which the contact was derived. In Figure 8.2 we show the pressure in sphere packings compared to loaded springs.

Close to jamming where the temperature $T$ is low packings and networks have similar pressure. With increasing temperature the springs exhibit the expected decrease in pressure, while repulsive interactions in the packings lead to an increase in $p$. The pressure in packings scales like $T^{1 / 2}$. This is due to the one-sided repulsive interaction potential for which the sphere-overlap $\delta$ scales like squareroot of temperature [88]. The sphere-overlap in volume $\delta$ is directly related to the pressure [7]:

$$
p \sim \delta^{\alpha-1}
$$

For harmonic interactions we have $\alpha=2$, which then explains the scaling of the packing's pressure.

The regime where $p \propto T^{1 / 2}$ is defined by contacts which are lost due to heat- 

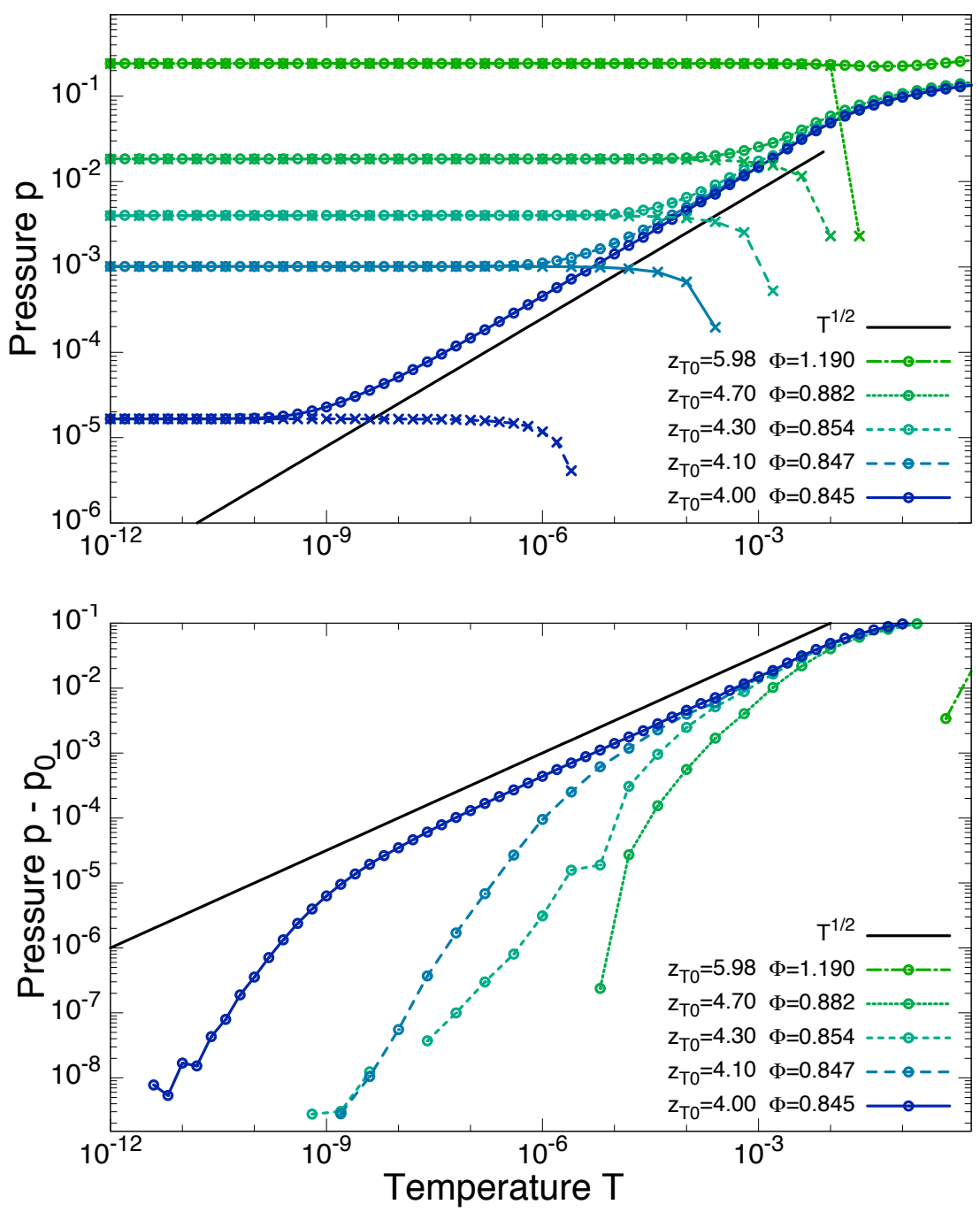

Figure 8.2: Upper panel: Pressure of sphere packings (circles) compared to the corresponding spring networks (crosses) as a function of temperature for coordination numbers at zero temperature $z_{T 0}$. Lower panel: pressure of packings compared to pressure at $T=0$. Each density corresponds to a coordination at zero temperature, which is conveniently compared to the coordination of networks where it is independent of $T$. For low temperatures close to jamming - packings and networks have similar pressure. With increasing temperature the springs exhibit the expected decrease in pressure, while repulsive interactions in the packings lead to an increase in $p$. The packing's pressure scales with temperature like $T^{1 / 2}$ (black line). System size $N=100$. 


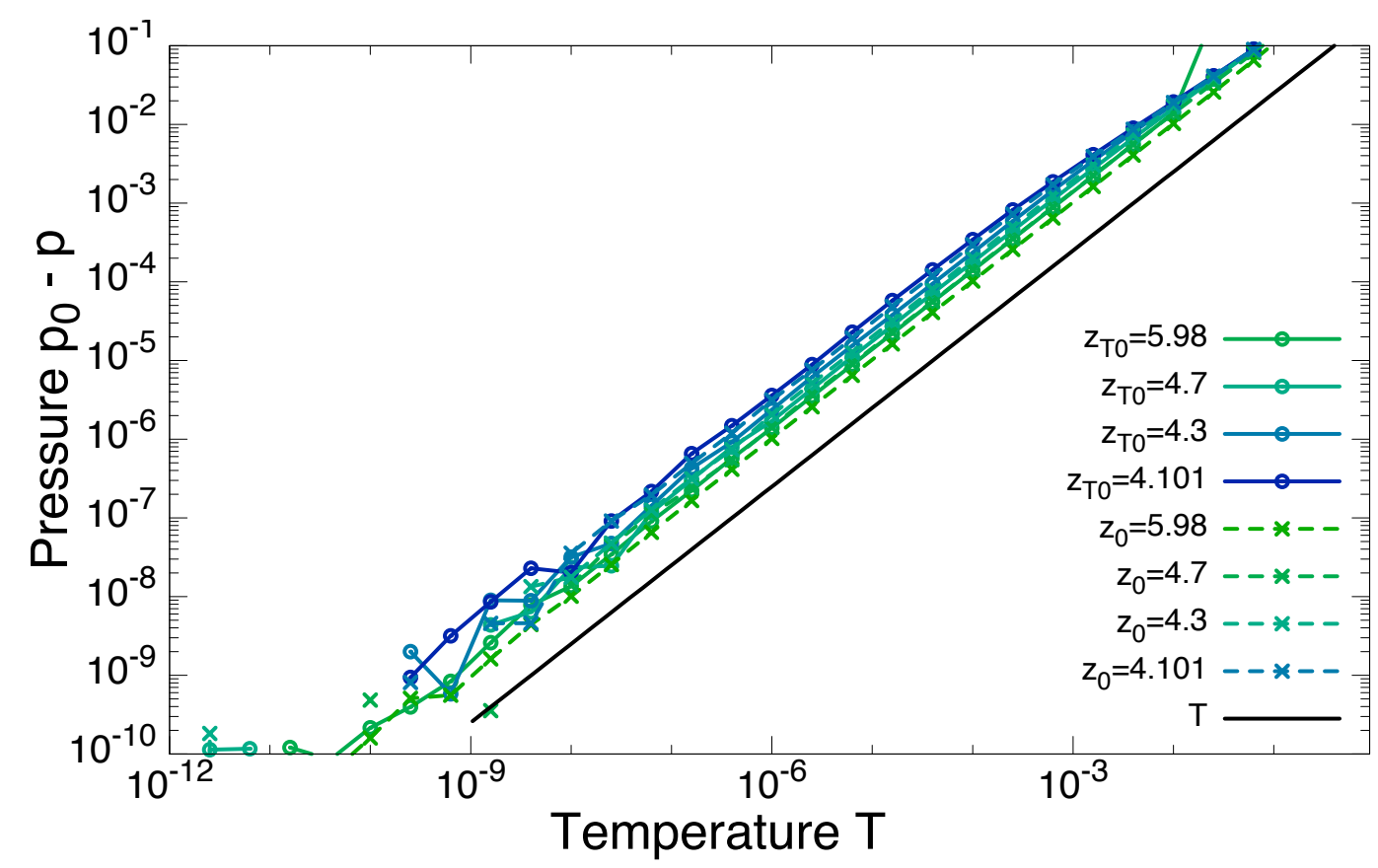

Figure 8.3: Pressure $p-p_{0}$ of loaded (circles) and relaxed (crosses) springs in NVT. (log-log) Both types of spring networks exhibit negative pressure linear with temperature $T$.

ing. This will be further discussed in section 8.2. Further increasing temperature leads to rearrangements and a less steep increase in pressure. At some point we will reach the limit of an ideal gas, where the virial pressure terms are constant and neglibile compared to pushes against the wall and the total pressure is proportional to temperature $T$.

In Figure 8.3 we show the pressure $p-p_{0}$ for fixed-volume in loaded and relaxed springs as a function of temperature $T$.

In networks contacts are permanent and have an attractive component. As expected - from the $N p T$ simulations and considerations - the networks contract. The scaling is of the pressure where temperature is negative and linear:

$$
p-p_{0} \sim-T
$$

This holds as long as there are no rearrangements: for low coordinations $(z=$ 4.101) and high temperatures $\left(T>10^{-3}\right)$ it can be seen in Figure 8.3 that the scaling becomes invalid. This is where the deplacement of vertices gets too large. Before that, springs stretch - instead of breaking contacts as packings would - 
and the attractive element in their interaction leads a decrease in pressure.

As a gedankenexperiment, the situation for jammed sphere packings should not be different from the corresponding networks with loaded springs as long as no contacts are broken. In both cases the same link-structure exists and interactions are repulsive. Hence, jammed sphere packings should exhibit a negative relative pressure $\Delta p=p-p_{0}$, too. In Figure 8.5 simulation data is shown for large coordination numbers.

Indeed, we find that packings contract for coordination numbers down to $z_{T 0}=$ 4.7, which is not to be understood as a universal value but as the limit that we determined with the simulations.

Considering that packings only exhibit repulsive contact interactions it is an intriguing result that they contract for a certain range of temperatures.

\subsection{Breaking of contacts}

Increasing the temperature leads to movements of spheres in a packing and results in broken bonds for certain finite temperatures. In order to understand this we consider the effective coordination number $z_{e f f}(T)$ of packings, which is the number of contacts averaged over snapshots of the system:

$$
z_{e f f}(T)=\frac{1}{t} \sum_{t} z_{t}(T)
$$

In this work we average over simulation time $t$ according to the described Monte Carlo sampling. The effective coordination number is a good observable to quantify the change in structure due to heating [88].

In Figure 8.6 we show the effective coordination compared to its value at zero temperature $z_{e f f} / z_{T 0}$.

One might think that as we increase $T$ the spheres in the volume explore more of their environment and form new contacts. This is not the case. The effective coordination number is lowered with increasing temperature [88]. Consistent with [88] $z_{\text {eff }} / z_{T 0}$ goes to $1 / 2$.

A change in the contact network affects the behaviour of the packing. Close to the jamming point this can be due to single links that break and results in a change of the mode spectrum [89].

The contraction we see for jammed packings happens far away from the jamming 


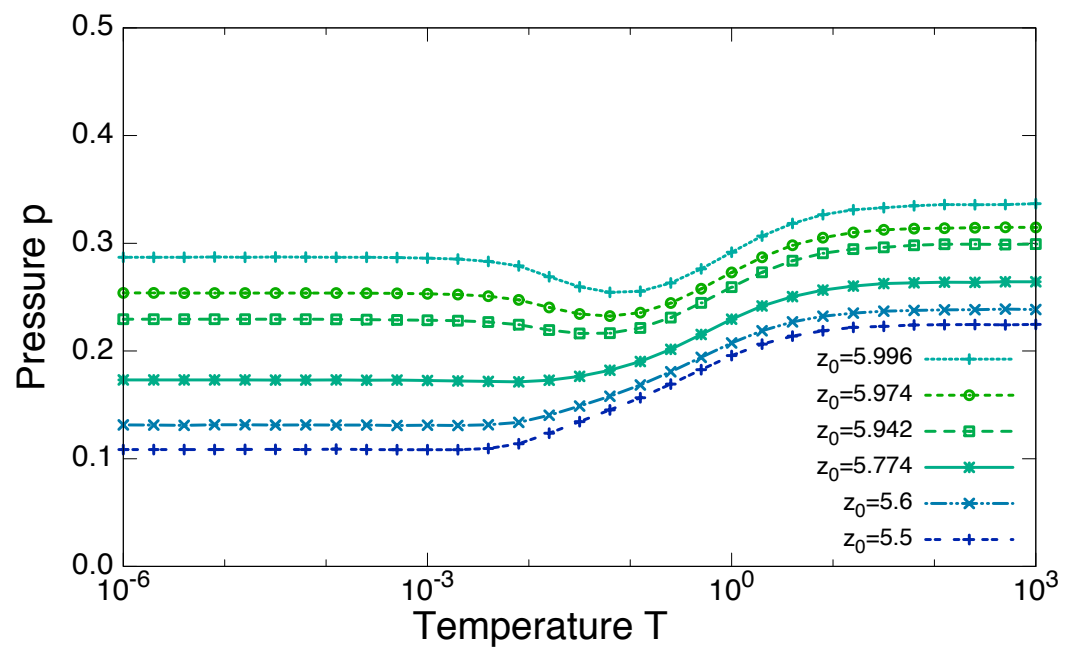

Figure 8.4: Pressure of sphere-packings as a function of temperature for different densities. Each density corresponds to a coordination at zero temperature. In order to compare more conveniently to results in spring networks we use $z_{0}$ to distinguish different sphere packings, too. Larger system size of $N=1000$.

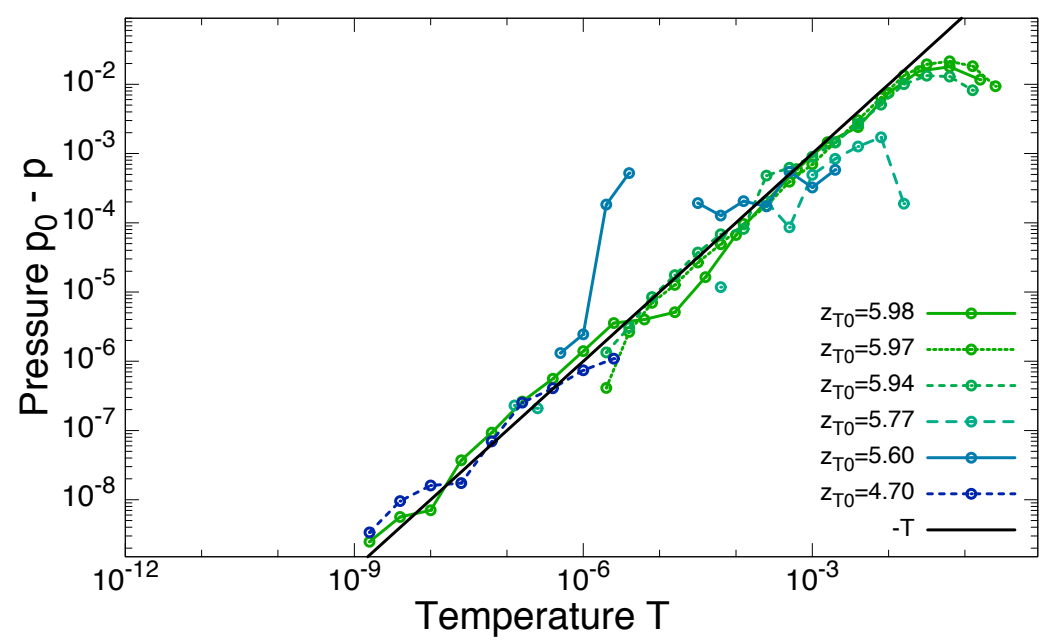

Figure 8.5: Pressure of sphere-packings compared to their pressure in the zero-temperature limes. The pressure decreases for low enough temperatures in which the contact network is close enough to the $T=0$ case. Hence, the sphere packings contract as the spring networks do. $z_{T 0}=4.70$ and $z_{T 0}=5.98$ is for a system of size $N=100$ with i.e. $10^{12}$ simulation steps. The values $z_{T 0}=5.60-5.97$ were conducted with $N=1000$ and $10^{10}$ steps. 


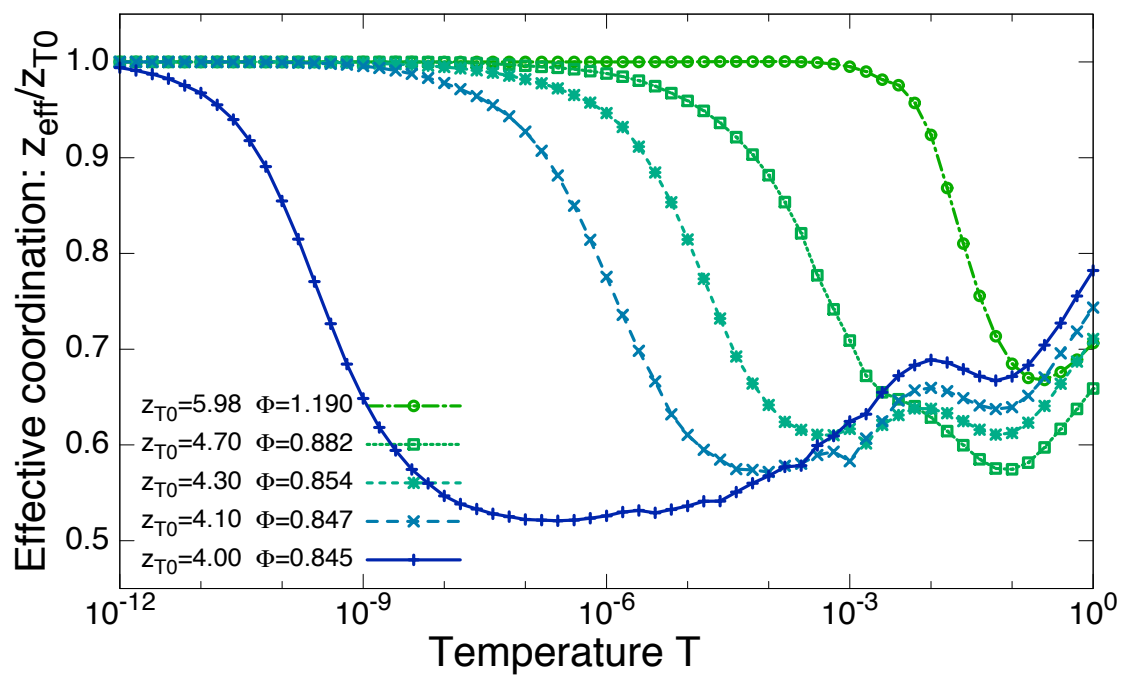

Figure 8.6: Effective coordination of sphere packings as a function of temperature compared to zero-temperature coordination $z_{T 0}$. The onset of the breaking contacts, which results in an increase of pressure, at certain temperature $T_{c}\left(z_{0}\right)$ is clearly visible. $T_{c}\left(z_{0}\right)$ increases with $z_{0}$.

point, which means an excess of links. Spring networks have a fixed contact network. Hence, thermal fluctuations cannot break links and the mode spectrum is kept intact. Spring networks show contraction over a large range of temperatures and for coordinations close to isostaticity as the mode analysis predicts.

So the key is in understanding how many links might be broken before the predictions fail. This needs to be investigated further.

\subsection{Brief note: the handling of rattlers}

We studied the effect of rattlers on the pressure of jammed sphere packings. Trivially a spring network does not contain rattlers and the decrease in pressure with $T$ is much more prevalent approaching the isostatic point. Approaching the isostatic point, however, the number of rattlers increases, see 2.3.

This is one of the reasons we investigated the effect of rattlers. For this we compared three situations: keeping rattlers, excluding rattlers and keeping them but excluding their contribution to pressure during the simulation.

Even though one might think that excluding rattlers might lead to decreasing pressure due to the freed volume, this is not the case. 


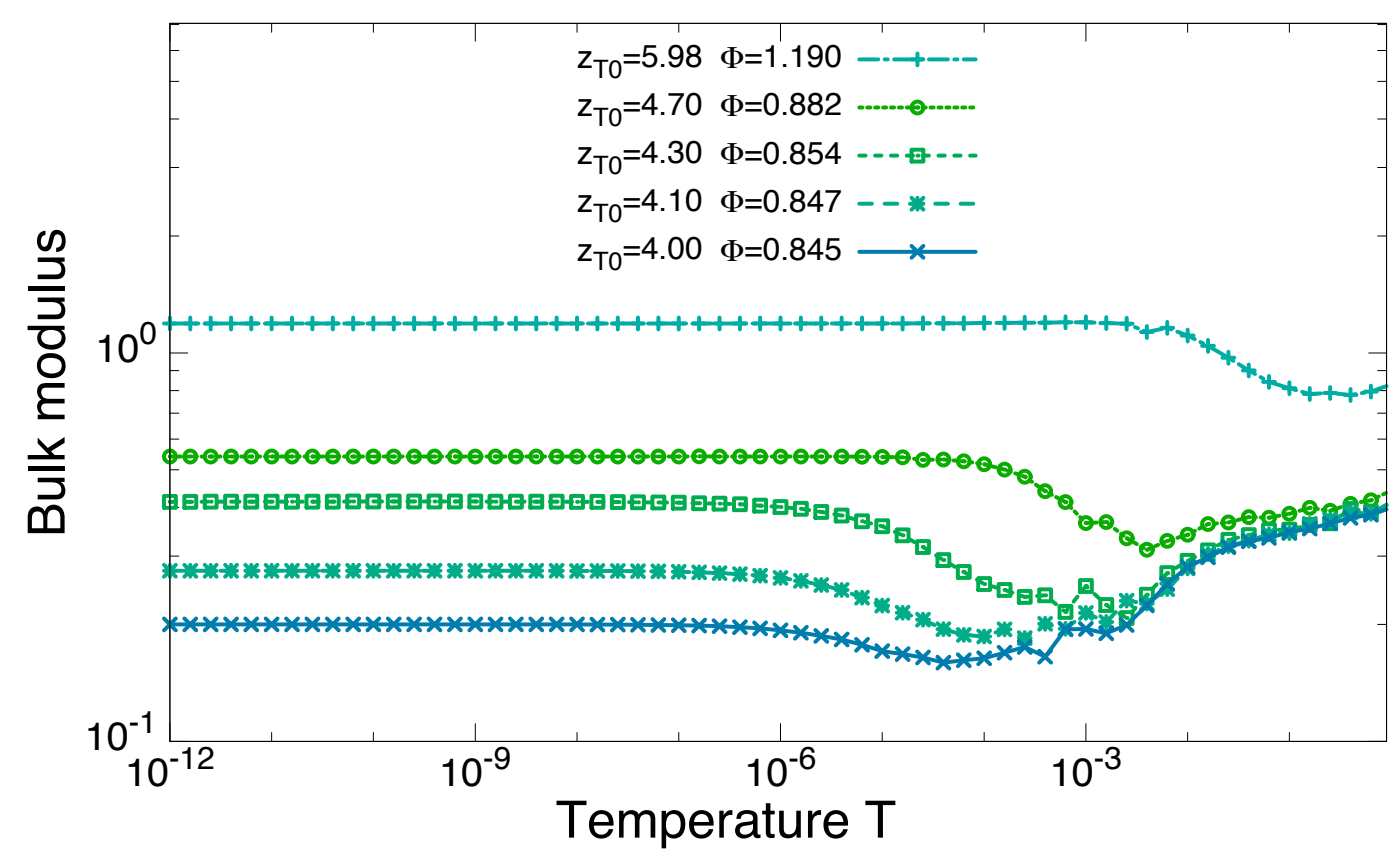

Figure 8.7: The bulk modulus of sphere packings. We see a decrease with rising temperature.

Overall we conclude that the effect of rattlers on the development of the pressure is neglibile.

\subsection{Bulk modulus}

We consider the bulk modulus of jammed packings, see Figure 8.7. It softens with increasing temperature which might because of contacts breaking due to temperature.

We want to probe if we see the same scaling behaviour as in the case of jammed spring networks in $N p T$. For this we apply the scaling to our data and show it in Figure 8.8.

Very cautious we may argue that the scaling might hold over a regime of finite temperatures and that we find the same scaling as in Chapter 5.2. Interestingly the scaling might hold even though many contacts are broken already due to the rise in pressure. This relation needs to be clarified especially as a function of the contact number $\Delta z$. 


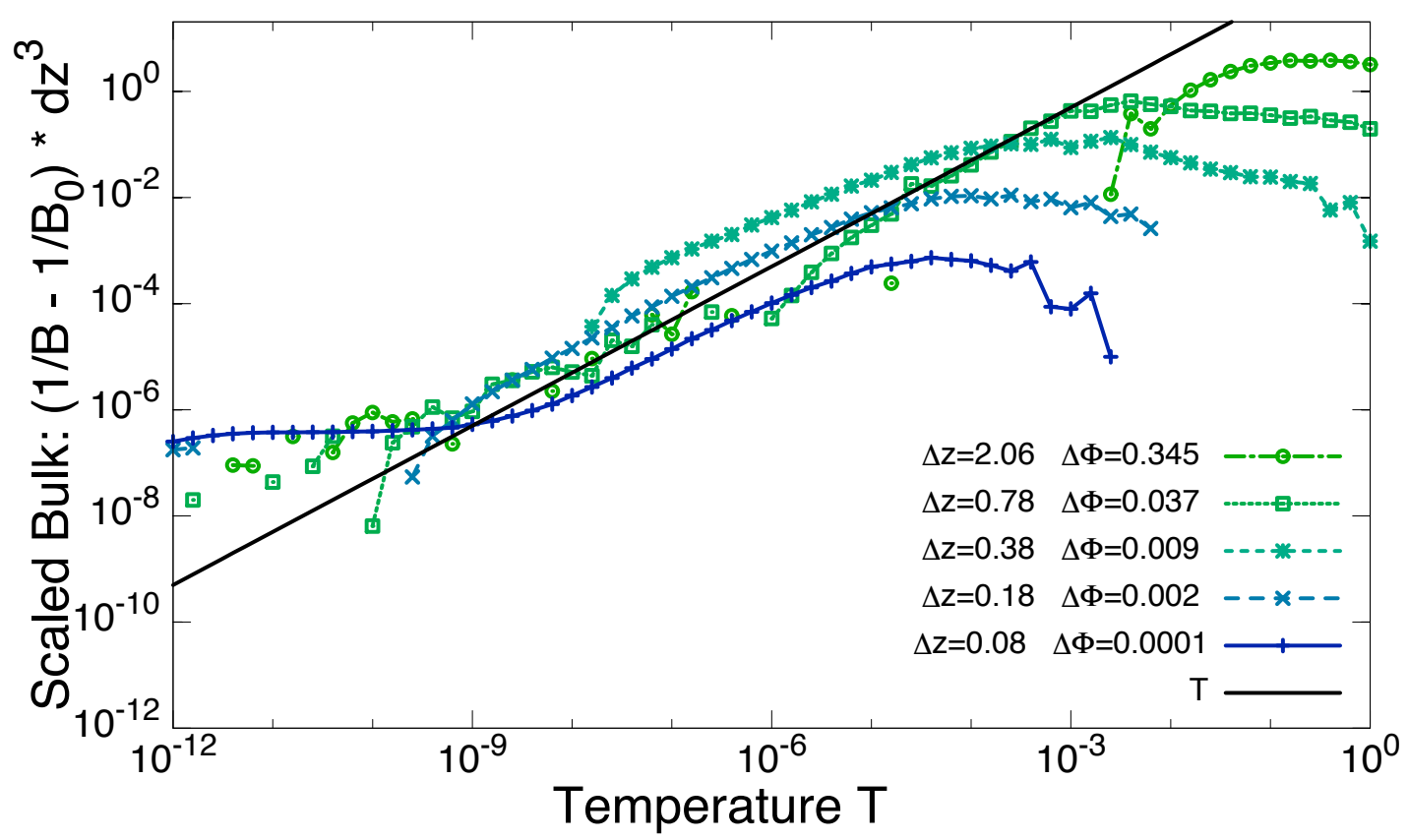

Figure 8.8: Scaling of bulk modulus with temperature T. It seems that the same scaling as in the spring networks can be recovered. Simulations for lower temperatures will further support the scaling relation.

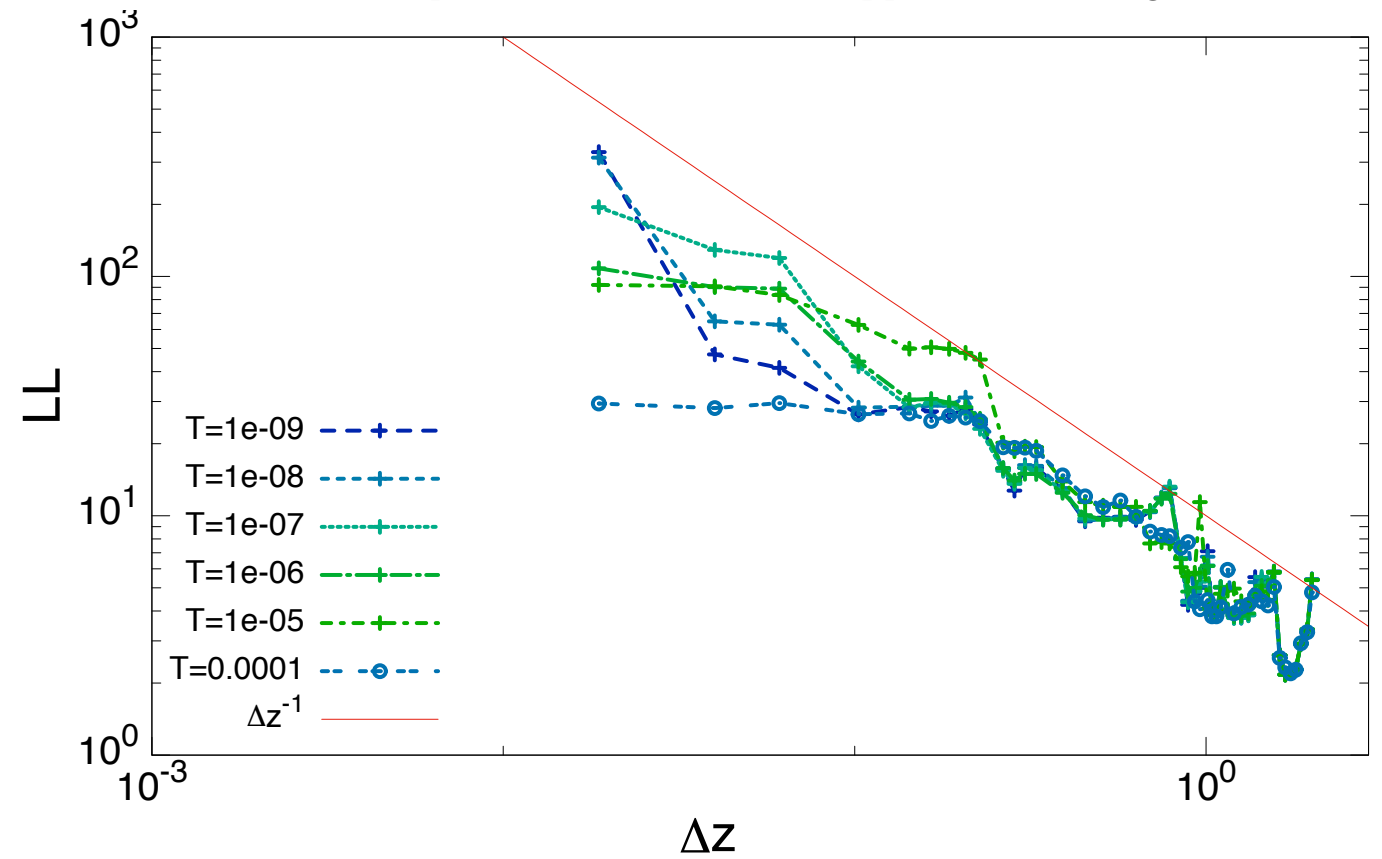

Figure 8.9: Scaling of localisation length with $\Delta z$. It seems that the same scaling like in the spring networks can be recovered: $L L \sim \Delta z^{-1}$. 


\subsection{Localisation length}

As a final quantity we probe the localisation length. In Figure 8.9 have data for $L L$ as a function of coordination number for different temperatures.

The localisation length shows the scaling $\sim \Delta z^{-1}$ which we already found in the spring networks. This is reassuring and maybe the least surprising because the movement of spheres in the packing should depend the least on contact forces, after all the volume in which they quite small and for low temperatures defined by the pressure at $T=0$. 


\section{Discussion}

Spring networks are an interesting and meaningful model system for probing the influence of temperature on the jamming transition. In this chapter we recap the tentpole results of our work and draft trajectories of future complementing and continuative research.

\subsection{Spring networks show negative thermal expansion}

Jammed spring networks contract at finite temperatures. We find that in the case of vanishing tension that the relative contraction $1-\frac{A}{A_{0}}$ scales linearly with temperature $T$ and inversely with coordination number, $\Delta z^{-1}$. Hence, the negative thermal expansion coefficient NTE in the isothermal-isobaric ensemble scales like $\Delta z^{-1}$ at vanishing tension $\tau=0$. To the best of our knowledge this statement has not been made yet.

\subsection{Spring networks exhibit a finite bulk modulus above the jamming point which softens with increasing temperature}

Athermal jammed spring networks maintain a finite bulk modulus $B$ until the jamming point $J$, where $B$ drops sharply to zero [2]. We confirm this behaviour and add that the bulk modulus softens with increasing temperature, which leads to the smearing of the otherwise sharp transition. For vanishing tension the inverse bulk modulus or compressibility relative to the athermal compressibility relates to temperature $T$ and coordination $\Delta z$ as:

$$
\frac{1}{B}-\frac{1}{B_{0}}=\frac{T}{\Delta z^{3}}
$$




\section{Discussion}

It would have been imaginable that with temperature-induced contraction the system will become stiffer. This is not the case.

\subsection{The shear modulus of spring networks stiffens with temperature}

Shear moves - like temperature - activate the anomalous modes of a jammed system $[62,90]$. Hence they determine the non-affine shear response of the material. This is known as shear dilatancy [9]. We considered the shear modulus in the canonical ensemble at finite temperatures. In the $N p T$ ensemble temperature leads to contraction. By definition this cannot happen in the canonical ensemble, where the volume is kept fixed. The resulting internal pressure leads to shear stiffening with temperature $T$ :

$$
G \approx T^{1 / 3} \Delta z^{-1}
$$

\subsection{The characteristics of spring networks are directly connected to their anomalous modes}

For the relative area contraction, the bulk modulus, the localisation length and the shear modulus we found scalings with simple exponents. The collapse on the master curve is in good agreement with the data for large ranges of temperatures $T$, distance to isostaticity $\Delta z$ and tension $\tau$.

The scalings found in the simulations are consistent with the predictions derived from the analysis of anomalous modes [61] - and vice versa. This is further support for the mode spectrum explained in $[28,29]$ being correct for finite temperatures. Furthermore, it punctuates the importance of non-affine contributions in amorphous media.

\subsection{Negative thermal expansion is a feature of most spring networks}

From the spring networks we explored we tentatively draw the conclusion that negative thermal expansion is a general feature of Hookean spring networks. The networks we considered were locally connected, which seems reasonable when 
having (amorphous) matter in mind. As a side note let us add that an early study of networks with completely randomly crossing Hookean springs showed these to be nonrigid on small deformations [83].

Tessier found traces of negative thermal expansion behaviour in fourfold connected networks [66] and Lammert [84] for the case of triangulated networks. We confirm this characteristic for pruned jammed spring networks - often called random networks $[2,64,65]$ - and pruned triangulated networks.

\subsection{The structure of spring networks strongly affects their mechanical features}

As pointed out, networks are often pruned to study how they behave with decreasing distance from the isostatic point. From Ellenbroek et al. [2] we know that the bulk modulus for random networks decreases and becomes zero at the isostatic point while it remains finite until $\Delta z=0$ in the case of jammed spring networks. We can confirm the scaling of $B \sim \Delta z^{1.5}[64]$ in the case of pruned networks. On a qualitative scale we see no strong temperature dependence of the pruned networks while jammed spring networks show the characteristic smearing of $B$ with $T$. Hence, when considering thermal fluctuations pruned networks and jammed spring networks show different behaviour.

This leaves the shear modulus as a comparable elastic modulus for jammed spring networks vs. pruned networks [2]. Dennison et al. [91] studied the shear modulus $G$ of pruned triangulated networks at finite temperatures. Their scaling variable is comparable to what we find in jammed spring networks: $\Delta z^{-2.8} T$. Their shear modulus for zero temperature is found to depend on coordination as $G \sim \Delta z^{1.4}$. In contrast, our results suggest that $G(T=0) \sim \Delta z$. Hence we want to stress the point that when studying the $\Delta z$-dependence of elastic moduli of networks with temperature it makes a difference if they are directly obtained from jammed packings or not.

In the case of the shear modulus also the protocol from which packings are obtained needs to be considered closely. From $[10,16,92]$ we learn that most jammed packings close to isostaticity obtained from compression-only protocols are unstable to shear. As a remedy they introduce a shear-stabilizing protocol. How the shear modulus develops for networks obtained from such a protocol will be subject of further studies.

One type of network we did not consider are fourfold networks with added links [93]. This could easily be added to this study. 


\subsection{Jammed spring networks expose a contraction-collapse phase transition with strong indication of a critical point}

We studied the phase transition from the regime where jammed spring networks contract to the regime where temperature becomes so high that the system collapses. This is definitely the case for $\tau>1.0$ - remember that this tension is larger than the spring constant. We find that the positive tension at certain values stabilizes the system so that the collapse transition shifts to higher temperatures. As a consequence the relative contraction is larger when the collapse happens at a certain temperature $T_{c}(\Delta z, \tau)$.

We have strong indications of a first-order phase transition with a critical point in the $p-T$-plane which is probably shared for differently coordinated networks. In $[74,94]$ triangulated networks were studied. They find a collapse transition as well but no critical point in the case of $N p T$ simulations [94]. Perhaps this has to do with the anisotropy of hexagonal networks: Their networks collapse from $C 6$ to $C 2$ while jammed spring networks are isotropic and should collapse evenly in both dimensions. They do so to the best of our knowledge on a qualitative scale. This point needs to be further investigated and quantified. Additionally, finite-size effects need to be checked and blocked in order to determine the exact position of the criticial point and the phase boundaries.

The phase diagram connects back to the jamming diagram Figure 1.3 [5], even though we do not consider active shearing as the proposed diagram does. It is an example of a phase diagram for a real jammed system.

\subsection{Spring networks contract, jammed packings do not always expand}

We studied the effect of temperature on jammed packings and in particular compared the results to those in spring networks in the canonical ensemble. Spring networks contract, or rather exhibit negative pressure in the canonical ensemble. Packings expand, but they do so close to the isostatic point and for large temperatures $T$. Packings far away from the jamming point show negative pressure before a certain number of links are broken. Then they expand, too.

This effect - to the best of our knowledge - has not been observed yet. It is especially interesting because in contrast to the spring networks the interactions in the jammed packings are merely repulsive. Within the limits of low temperatures, however, it seems that the main difference to the networks is that their link 
structure is persistent. And hence their mode spectrum.

This leads us to our last statement which predictions we can transfer from spring networks to jammed packings.

\subsection{Spring networks help in understanding thermalized jammed packings}

We could show that there is a relation between jammed packings and their spring networks at finite temperatures. This seems to go beyond the contraction for certain rather high packing fractions.

It might be conceivable that the prediction from spring networks for the bulk modulus holds over a range of temperatures for packings, too. One might draw the conclusion that the bulk modulus is less sensitive to losing contacts.

As a third observable we checked the localisation length for jammed packings and its dependence on $\Delta z$. The result gave the strongest agreement with the predictions and the simulations of spring networks.

The shear modulus is another obvious candidate to be checked. If we see shear stiffening behaviour like in the spring networks shear might even lead to new contacts. However, we need to see how stable the packings we use are actually to shear movements $[10,16,92]$. 



\section{Outlook}

This study explored many interesting aspects of jammed spring networks and jammed packings subject to finite temperature. Yet many questions are left open - or opened up when details were investigated.

The exact conditions for contraction of packings should be subject of further research. More importantly, a detailed study of scalings in packings is of high interest. Results indicate that there might be more connections between spring networks and jammed packings than initially expected. It is crucial to understand how many links can be broken before predictions fail. It seems likely that this number is different for different properties of the system.

An obvious extension of this work is to consider packings also in the isothermalisobaric ensemble which has not been done so far. It would be interesting to probe different potentials, i.e. Hertzian or attractive. In the latter, bonds do not break as easily, as in the repulsive case, which should make comparisons easier.

The jammed spring networks we presented provide a model system for a material in dependence of temperature, coordination and tension. It would be interesting to find out if this can model the behaviour of real materials. After all, the properties we showed are very interesting: negative thermal expansion, shear stiffening and a finite bulk modulus above the jamming transition which softens with temperature. Negative thermal expansion - as discussed in the introduction - is attributed to anomalous modes and prevalent in many materials.

It would be interesting to see if it is possible to prepare a packing in the laboratory so that it exhibits contraction as we see it. This is an interesting property for engineering purposes. We showed that jammed spring networks provide an interesting model system with realistic mechanical properties which is now well understood. Other types of networks exhibit negative thermal expansion as well, but are less stable towards collapse with temperature and expose a rather unrealistic behaviour of the bulk modulus.

Furthermore, it would be interesting to see if some of the results can be related to glasses, i.e. silica [95-97]. Even though jammed spring networks exhibit negative thermal expansion, as silica does, its bulk modulus stiffens with temperature. This is in contrast to the networks we considered, hence it does not seem that there is an easy connection. 


\section{Outlook}

Instead of searching for materials with similar features, maybe our insights will help to engineer new materials. It might be useful to be able to finely tune negative thermal expansion. In some of the simulations for certain packings we saw that the pressure for packings was not changing over a range of temperatures. This effect could not be reproduced in a resilient manner under clear conditions so it might just be an artefact of the energy minimisation. On the other hand it gives a hint that zero thermal expansion (ZTE) [98] is an interesting topic of research. What are the conditions for zero thermal expansion in the context of jamming and the connectivity of a packing or a network? Can a ZTE material be designed? Certainly, this is interesting for many engineering purposes.

It seems that the presented research leaves more questions open than it answered, which - with all due humbleness - is not a bad sign. 


\section{Bibliography}

[1] M van Hecke. Jamming of soft particles: geometry, mechanics, scaling and isostaticity. Journal of Physics: Condensed Matter, 22(3):033101, 2010.

[2] W. G. Ellenbroek, Z. Zeravcic, W. van Saarloos, and M. van Hecke. Nonaffine response: Jammed packings vs. spring networks. EPL (Europhysics Letters), 87(3):34004, 2009.

[3] Dirk Helbing, Illes Farkas, and Tamas Vicsek. Simulating dynamical features of escape panic. Nature, 407(6803):487-490, 092000.

[4] Kai Nagel and Michael Schreckenberg. A cellular automaton model for freeway traffic. J. Phys. I France, 2(12):2221-2229, 1992.

[5] Andrea J. Liu and Sidney R. Nagel. Nonlinear dynamics: Jamming is not just cool any more. Nature, 396(6706):21-22, 111998.

[6] Demtroeder. Experimentalphysik 1: Mechanik und Wärme. Springer, Berlin Heidelberg, 2003.

[7] Alexander O.N. Siemens and Martin van Hecke. Jamming: A simple introduction. Physica A: Statistical Mechanics and its Applications, 389(20):4255 - 4264, 2010. Proceedings of the 12th International Summer School on Fundamental Problems in Statistical Physics.

[8] Andrea J. Liu and Sidney R. Nagel. The jamming transition and the marginally jammed solid. Annual Review of Condensed Matter Physics, 1(1):347-369, 2010.

[9] Brian P. Tighe. Shear dilatancy in marginal solids. Granular Matter, 16(2):203-208, 2014.

[10] Simon Dagois-Bohy, Brian P. Tighe, Johannes Simon, Silke Henkes, and Martin van Hecke. Soft-sphere packings at finite pressure but unstable to shear. Phys. Rev. Lett., 109:095703, Aug 2012.

[11] Ludovic Berthier and Thomas A. Witten. Glass transition of dense fluids of hard and compressible spheres. Phys. Rev. E, 80:021502, Aug 2009.

[12] Zexin Zhang, Ning Xu, Daniel T. N. Chen, Peter Yunker, Ahmed M. Alsayed, Kevin B. Aptowicz, Piotr Habdas, Andrea J. Liu, Sidney R. Nagel, and 


\section{BIBLIOGRAPHY}

Arjun G. Yodh. Thermal vestige of the zero-temperature jamming transition. Nature, 459(7244):230-233, 052009.

[13] Corey S. O’Hern, Leonardo E. Silbert, Andrea J. Liu, and Sidney R. Nagel. Jamming at zero temperature and zero applied stress: The epitome of disorder. Phys. Rev. E, 68:011306, Jul 2003.

[14] Corey S. O'Hern, Stephen A. Langer, Andrea J. Liu, and Sidney R. Nagel. Random packings of frictionless particles. Phys. Rev. Lett., 88:075507, Jan 2002.

[15] F. Bolton and D. Weaire. Rigidity loss transition in a disordered 2d froth. Phys. Rev. Lett., 65:3449-3451, Dec 1990.

[16] Carl P. Goodrich, Andrea J. Liu, and Sidney R. Nagel. Finite-size scaling at the jamming transition. Phys. Rev. Lett., 109:095704, Aug 2012.

[17] Corey S. O'Hern, Leonardo E. Silbert, Andrea J. Liu, and Sidney R. Nagel. Jamming at zero temperature and zero applied stress: The epitome of disorder. Phys. Rev. E, 68:011306, Jul 2003.

[18] T. S. Majmudar, M. Sperl, S. Luding, and R. P. Behringer. Jamming transition in granular systems. Phys. Rev. Lett., 98:058001, Jan 2007.

[19] D. J. Durian. Foam mechanics at the bubble scale. Phys. Rev. Lett., 75:47804783, Dec 1995.

[20] JC Maxwell. 598. Philosophical Magazine 27, 1865.

[21] Ellák Somfai, Martin van Hecke, Wouter G. Ellenbroek, Kostya Shundyak, and Wim van Saarloos. Critical and noncritical jamming of frictional grains. Phys. Rev. E, 75:020301, Feb 2007.

[22] Mitch Mailman, Carl F. Schreck, Corey S. O'Hern, and Bulbul Chakraborty. Jamming in systems composed of frictionless ellipse-shaped particles. Phys. Rev. Lett., 102:255501, Jun 2009.

[23] Vincent J. Langlois, Stefan Hutzler, and Denis Weaire. Rheological properties of the soft-disk model of two-dimensional foams. Phys. Rev. E, 78:021401, Aug 2008.

[24] Peter Debye. Zur theorie der spezifischen wärme. Annalen der Physik. 4. Folge, Band 39, S. 789-839. Hg. von Wilhelm Wien und Max Planck., 1912.

[25] Leonardo E. Silbert, Andrea J. Liu, and Sidney R. Nagel. Vibrations and diverging length scales near the unjamming transition. Phys. Rev. Lett., 95:098301, Aug 2005.

[26] A. J. Liu, S. R. Nagel, W. van Saarloos, and M. Wyart. The jamming scenario - an introduction and outlook. Dynamical heterogeneities in glasses, colloids, 
and granular media, Oxford Univ Press, Oxford, UK, June 2010.

[27] Wouter G. Ellenbroek, Ellák Somfai, Martin van Hecke, and Wim van Saarloos. Critical scaling in linear response of frictionless granular packings near jamming. Phys. Rev. Lett., 97:258001, Dec 2006.

[28] M. Wyart, S. R. Nagel, and T. A. Witten. Geometric origin of excess lowfrequency vibrational modes in weakly connected amorphous solids. EPL (Europhysics Letters), 72(3):486, 2005.

[29] Matthieu Wyart. On the rigidity of amorphous solids. Dissertation, 2005.

[30] Leonardo E. Silbert, Andrea J. Liu, and Sidney R. Nagel. Normal modes in model jammed systems in three dimensions. Phys. Rev. E, 79:021308, Feb 2009 .

[31] Ning Xu, Vincenzo Vitelli, Matthieu Wyart, Andrea J. Liu, and Sidney R. Nagel. Energy transport in jammed sphere packings. Phys. Rev. Lett., 102:038001, Jan 2009.

[32] V. Trappe, V. Prasad, Luca Cipelletti, P. N. Segre, and D. A. Weitz. Jamming phase diagram for attractive particles. Nature, 411(6839):772-775, 06 2001.

[33] Giorgio Parisi. The discoveries of slowness. Physik Journal 10 (2011) Nr. 8/9, pages 1617-9437/11/0909-29, 2011.

[34] Andreas Heuer, Michael Kastner, Alexander Karl Hartmann, and Martin Weigel. Wanderungen in energielandschaften. Physik Journal 9 (2010) Nr. 12, 2010.

[35] W. A. Phillips. Amorphous Solids, Low Temperature Properties. Springer, Berlin, 1981.

[36] A. P. Sokolov, U. Buchenau, W. Steffen, B. Frick, and A. Wischnewski. Comparison of raman- and neutron-scattering data for glass-forming systems. Phys. Rev. B, 52:R9815-R9818, Oct 1995.

[37] Charles Kittel. Introduction to Solid State Physics, 8th Edition. Wiley, 2004.

[38] Siegfried Hunklinger. Festkörperphysik. De Gruyter Oldenbourg, 2006.

[39] R. C. Zeller and R. O. Pohl. Thermal conductivity and specific heat of noncrystalline solids. Phys. Rev. B, 4:2029-2041, Sep 1971.

[40] Carolina Brito and Matthieu Wyart. Geometric interpretation of previtrification in hard sphere liquids. The Journal of Chemical Physics, 131(2):-, 2009.

[41] Carolina Brito and Matthieu Wyart. Heterogeneous dynamics, marginal sta- 


\section{BIBLIOGRAPHY}

bility and soft modes in hard sphere glasses. Journal of Statistical Mechanics: Theory and Experiment, 2007(08):L08003, 2007.

[42] Kostya O Trachenko, Martin T Dove, Mark J Harris, and Volker Heine. Dynamics of silica glass: two-level tunnelling states and low-energy floppy modes. Journal of Physics: Condensed Matter, 12(37):8041, 2000.

[43] Kostya Trachenko, Martin T. Dove, Vadim Brazhkin, and F. S. El'kin. Network rigidity and properties of $\mathrm{sio}_{2}$ and $\mathrm{geO}_{2}$ glasses under pressure. Phys. Rev. Lett., 93:135502, Sep 2004.

[44] I P Swainson and M T Dove. Molecular dynamics simulation of alpha - and beta -cristobalite. Journal of Physics: Condensed Matter, 7(9):1771, 1995.

[45] Matthieu Wyart, Leonardo E. Silbert, Sidney R. Nagel, and Thomas A. Witten. Effects of compression on the vibrational modes of marginally jammed solids. Phys. Rev. E, 72:051306, Nov 2005.

[46] Matthieu Wyart. Rigidity-based approach to the boson peak in amorphous solids: from sphere packing to amorphous silica. ArXiv e-prints, 062008.

[47] A. I. Chumakov, G. Monaco, A. Monaco, W. A. Crichton, A. Bosak, R. Rüffer, A. Meyer, F. Kargl, L. Comez, D. Fioretto, H. Giefers, S. Roitsch, G. Wortmann, M. H. Manghnani, A. Hushur, Q. Williams, J. Balogh, K. Parliński, P. Jochym, and P. Piekarz. Equivalence of the boson peak in glasses to the transverse acoustic van hove singularity in crystals. Phys. Rev. Lett., 106:225501, May 2011.

[48] Ke Chen, Wouter G. Ellenbroek, Zexin Zhang, Daniel T. N. Chen, Peter J. Yunker, Silke Henkes, Carolina Brito, Olivier Dauchot, Wim van Saarloos, Andrea J. Liu, and A. G. Yodh. Low-frequency vibrations of soft colloidal glasses. Phys. Rev. Lett., 105:025501, Jul 2010.

[49] Georg Maret. Zweidimensionale festkörper. Physik Journal 10 (2011) Nr. 8/9, pages 1617-9437/11/0909-35,, 2011.

[50] J. B. Brown. Thermodynamics of a rubber band. American Journal of Physics, 31(5):397-397, 1963.

[51] Uni Köln (Autoren unbekannt). Gummielastizität - versuche im physikalischchemischen praktikum für fortgeschrittene. http://strey.pc.uni-koeln.de/ fileadmin/user_upload/Download/Gummielastizitaet_final_12052009.pdf, 2009.

[52] Xingyuan Shen, Christopher Viney, Erin R. Johnson, Changchun Wang, and Jennifer Q. Lu. Large negative thermal expansion of a polymer driven by a submolecular conformational change. Nat Chem, 5(12):1035-1041, 122013.

[53] Volker Heine, Patrick R. L. Welche, and Martin T. Dove. Geometrical origin 
and theory of negative thermal expansion in framework structures. Journal of the American Ceramic Society, 82(7):1793-1802, 1999.

[54] J. Paul Attfield. Condensed-matter physics: A fresh twist on shrinking materials. Nature, 480(7378):465-466, 122011.

[55] Benjamin K. Greve, Kenneth L. Martin, Peter L. Lee, Peter J. Chupas, Karena W. Chapman, and Angus P. Wilkinson. Pronounced negative thermal expansion from a simple structure: Cubic scf3. Journal of the American Chemical Society, 132(44):15496-15498, 2010. PMID: 20958035.

[56] Chen W. Li, Xiaoli Tang, J. A. Muñoz, J. B. Keith, S. J. Tracy, D. L. Abernathy, and B. Fultz. Structural relationship between negative thermal expansion and quartic anharmonicity of cubic $\operatorname{scf}_{3}$. Phys. Rev. Lett., 107:195504, Nov 2011.

[57] Mikael C. Rechtsman, Frank H. Stillinger, and Salvatore Torquato. Negative thermal expansion in single-component systems with isotropic interactions $\dagger$. The Journal of Physical Chemistry A, 111(49):12816-12821, 2007. PMID: 17988108 .

[58] Cora Lind. Two decades of negative thermal expansion research: Where do we stand? Materials, 5(6):1125-1154, 2012.

[59] G D Barrera, J A O Bruno, T H K Barron, and N L Allan. Negative thermal expansion. Journal of Physics: Condensed Matter, 17(4):R217, 2005.

[60] M. Wyart E. DeGiuli, E. Lerner. Theory of the jamming transition at finite temperature. arXiv, 2015.

[61] C. Buss, C. Heussinger, and O. Hallatschek. Thermalized connectivity networks of jammed packings. ArXiv e-prints, April 2016.

[62] Brian P. Tighe. Relaxations and rheology near jamming. Phys. Rev. Lett., 107:158303, Oct 2011.

[63] http://lammps.sandia.gov.

[64] M. Wyart, H. Liang, A. Kabla, and L. Mahadevan. Elasticity of floppy and stiff random networks. Phys. Rev. Lett., 101:215501, Nov 2008.

[65] Brian P. Tighe. Dynamic critical response in damped random spring networks. Phys. Rev. Lett., 109:168303, Oct 2012.

[66] Frédéric Tessier, David H. Boal, and Dennis E. Discher. Networks with fourfold connectivity in two dimensions. Phys. Rev. E, 67:011903, Jan 2003.

[67] Nicholas Metropolis and S. Ulam. The monte carlo method. Journal of the American Statistical Association, 44(247):335-341, 1949. PMID: 18139350. 


\section{BIBLIOGRAPHY}

[68] Finance with Monte Carlo. Ronald W. Shonkwiler, Springer-Verlag New York, 2013.

[69] M. E. J. Newman and G. T. Barkema. Monte Carlo Methods in Statistical Physics. Clarendon Press, 1999.

[70] Richard L.C. Vink. Monte Carlo Methods in Statistical Physics, Lecture Notes. Uni Goettingen, 2012.

[71] Nicholas Metropolis, Arianna W. Rosenbluth, Marshall N. Rosenbluth, Augusta H. Teller, and Edward Teller. Equation of state calculations by fast computing machines. The Journal of Chemical Physics, 21(6):1087-1092, 1953.

[72] W. W. Wood. Monte carlo calculations for hard disks in the isothermal isobaric ensemble. The Journal of Chemical Physics, 48(1):415-434, 1968.

[73] Daan Frenkel and Berend Smit. Understanding Molecular Simulation: From Algorithms to Applications (Second Edition). Academic Press, 978-0-12267351-1, 2002.

[74] Wolfgang Wintz, Ralf Everaers, and Udo Seifert. Mesh collapse in two-dimensional elastic networks under compression. J. Phys. I France, 7(9):1097-1111, 1997.

[75] Kurt Binder and Dieter W. Heermann. Monte Carlo Simulation in Statistical Physics. Springer, Berlin Heidelberg, 2002.

[76] MP Allen and DJ Tildesley. Computer Simulation of Liquids. Oxford University Press, 1991.

[77] H. Müller-Krumbhaar and K. Binder. Dynamic properties of the monte carlo method in statistical mechanics. Journal of Statistical Physics, 8(1):1-24, 1973.

[78] Fliessbach. Statistische Physik. Spektrum, 1995.

[79] Schwabl. Statistische Mechanik. Springer, 2006.

[80] T. C. Lubensky P. M. Chaikin. Principles of Condensed Matter Physics. Cambridge University Press, 2007.

[81] E M Landau, L D Lifshitz. Course of Theoretical Physics. ButterworthHeinemann, 2007.

[82] Computer Simulation in Physics and Engineering. Martin Oliver Steinhauser - De Gruyter, 2013.

[83] M. Kellomäki, J. Åström, and J. Timonen. Rigidity and dynamics of random spring networks. Phys. Rev. Lett., 77:2730-2733, Sep 1996. 
[84] Paul E. Lammert and Dennis E. Discher. Tethered networks in two dimensions: A low-temperature view. Phys. Rev. E, 57:4368-4374, Apr 1998.

[85] Donald Jacobs and Bruce Hendrickson. An algorithm for two dimensional rigidity percolation: The pebble game. Journal of Computational Physics, 137:346-365, 1997.

[86] D. J. Jacobs and M. F. Thorpe. Generic rigidity percolation: The pebble game. Phys. Rev. Lett., 75:4051-4054, Nov 1995.

[87] G. George Batrouni Michel Le Bellac, Fabrice Mortessagne. Equilibrium and Non-Equilibrium Statistical Thermodynamics. Cambridge University Press, 2004 .

[88] Thibault Bertrand, Carl F. Schreck, Corey S. O'Hern, and Mark D. Shattuck. Hypocoordinated solids in particulate media. Phys. Rev. E, 89:062203, Jun 2014.

[89] Carl F. Schreck, Thibault Bertrand, Corey S. O'Hern, and M. D. Shattuck. Repulsive contact interactions make jammed particulate systems inherently nonharmonic. Phys. Rev. Lett., 107:078301, Aug 2011.

[90] Brian P. Tighe. Critical viscoelastic response in jammed solids. arxiv.org/ abs/1205.2960, 2012.

[91] M. Dennison, M. Sheinman, C. Storm, and F. C. MacKintosh. Fluctuationstabilized marginal networks and anomalous entropic elasticity. Phys. Rev. Lett., 111:095503, Aug 2013.

[92] Eric Corwin. Getting into a proper jam. Physics, 5:97, Aug 2012.

[93] Anton Souslov, Andrea J. Liu, and T. C. Lubensky. Elasticity and response in nearly isostatic periodic lattices. Phys. Rev. Lett., 103:205503, Nov 2009.

[94] Dennis E. Discher, David H. Boal, and S. K. Boey. Phase transitions and anisotropic responses of planar triangular nets under large deformation. Phys. Rev. E, 55:4762-4772, Apr 1997.

[95] M. E. Fine, H. Van Duyne, and Nancy T. Kenney. Low-temperature internal friction and elasticity effects in vitreous silica. Journal of Applied Physics, 25(3):402-405, 1954.

[96] HAROLD T. SMYTH. Thermal expansion of vitreous silica. Journal of the American Ceramic Society, 38(4):140-141, 1955.

[97] R. Vacher, J. Pelous, F. Plicque, and A. Zarembowitch. Ultrasonic and brillouin scattering study of the elastic properties of vitreous silica between 10 and 300 k. Journal of Non-Crystalline Solids, 45(3):397 - 410, 1981.

[98] Peter Mohn. Materials science: A century of zero expansion. Nature, 
BIBLIOGRAPHY

400(6739):18-19, 071999. 
APPENDIX 



\section{Dependence on interaction potential}

\section{Anharmonic Interactions - quartic repulsion}

In order to probe the effect of anharmonic interactions we use the potential:

$$
V(d)= \begin{cases}\left(d-d_{0}\right)^{2}+Q\left(d-d_{0}\right)^{4}, & \text { if } d \leq d_{0} \\ \left(d-d_{0}\right)^{2}, & \text { if } d \geq d_{0},\end{cases}
$$

where $d$ is the length of the spring and $d_{0}$ its rest length. The parameter $\mathrm{Q}$ tunes the strength of the quartic term of the repulsion in units of the harmonic spring constant. Increasing $\mathrm{Q}$ makes the quartic term and hence the anharmonicities more and more dominant.

We want to test if the harmonic potential we used in our work is a prerequisite to observe the NTE (negative thermal expansion) effect.

\section{$\mathrm{Q}$ dependence}

Figure .1 shows the effect for different values of $\mathrm{Q}$ at different fixed temperatures.

\section{$T$ dependence}

The NTE effect prevails, perhaps even with the same $d z^{-1}$ scaling until a certain temperature-dependent strength of $Q$. The transition of the collapse shifts to lower temperatures for larger $Q$ factors. Yet the feature of negative thermal expansion prevails for anharmonicities defined by a factor $Q$ up to $10^{2}$ for temperatures below $T=10^{-5}$ for the case of $d z=5.96$. 

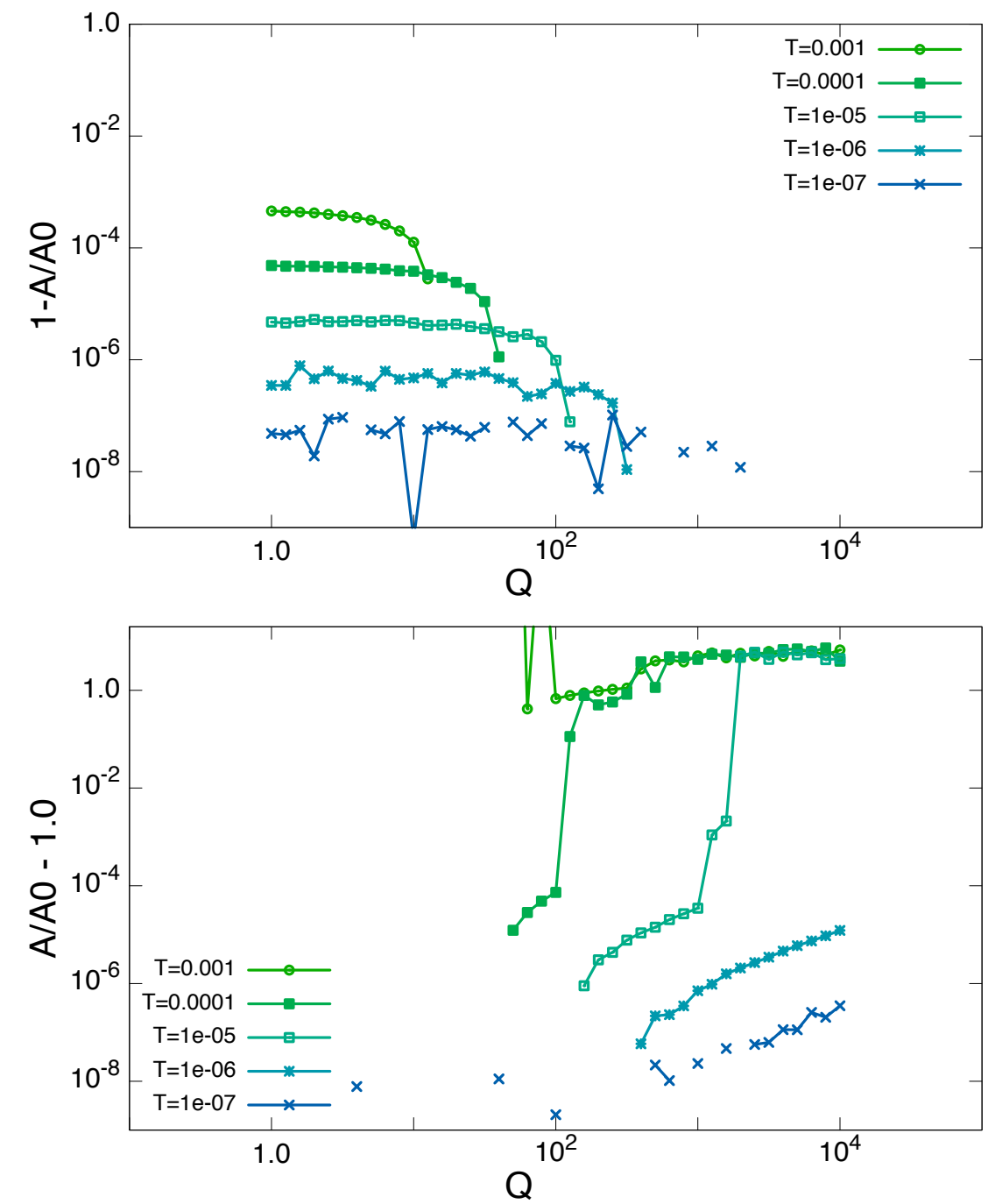

Figure .1: $\mathbf{z}=5$.96. Anharmonic potential with dependence on $Q . Q$ is the strength of repulsive quartic term. 

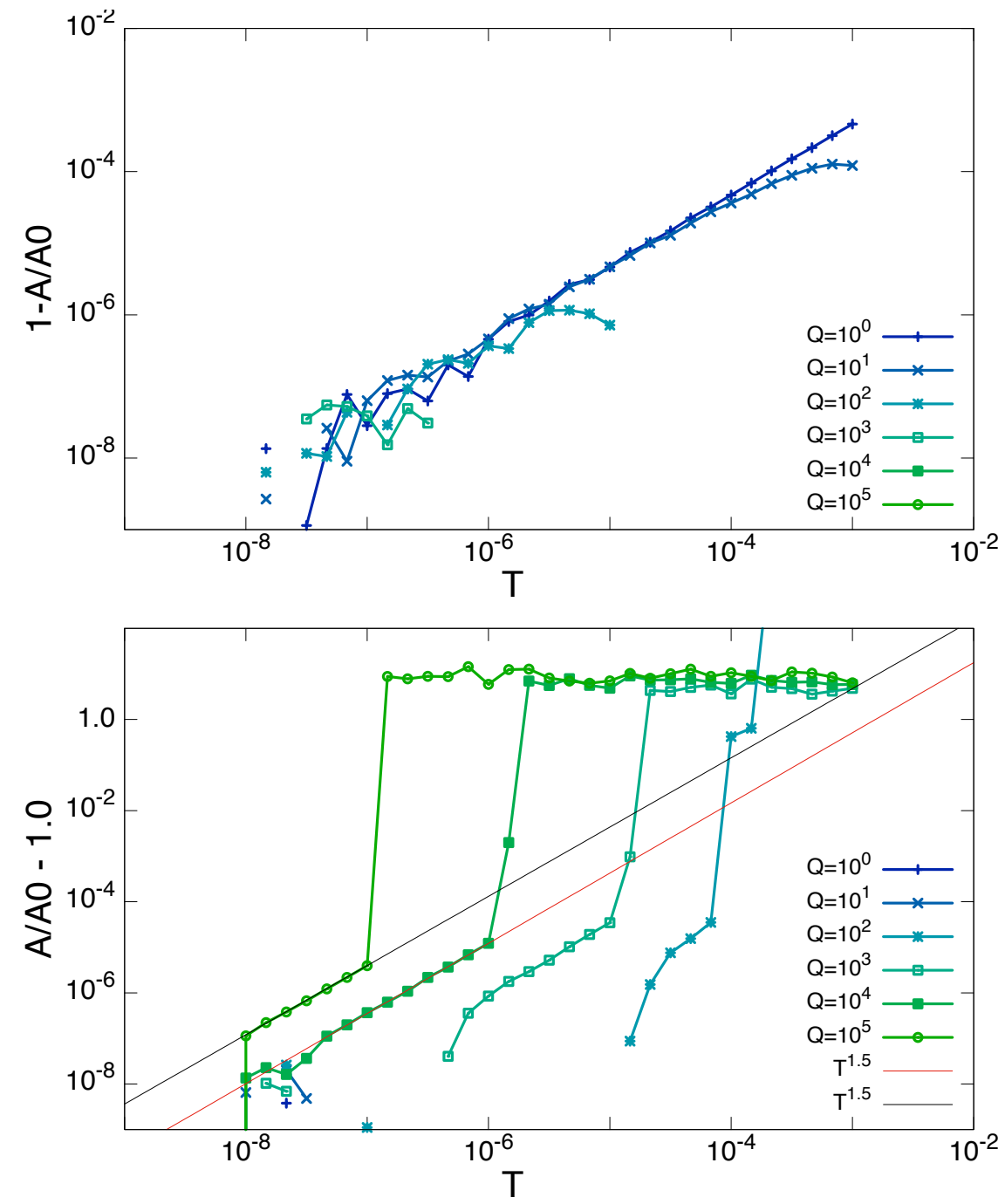

Figure .2: $\mathbf{z}=5$.96. Anharmonic potential with dependence on Temperature $T$. 


\section{Analytical approach}

We calculate the mean excitation for an oscillator $\langle x\rangle$ in the chosen, Q-dependent potential through the thermodynamic average:

$$
\langle x\rangle=\frac{\int_{-\infty}^{\infty} x e^{-\beta V(x)} d x}{\int_{-\infty}^{\infty} e^{-\beta V(x)} d x}
$$

Using the potential .1 inside the Boltzmann factor we can develop around harmonic contributions:

$$
e^{-\beta\left(x^{2}+Q x^{4}\right)}=e^{-\beta x^{2}} e^{-\beta Q x^{4}} \approx e^{-\beta x^{2}}\left(1-\beta Q x^{4} . .\right)
$$

Then we get for the numerator in $(.2)$ :

$$
\begin{aligned}
\int_{-\infty}^{\infty} x e^{-\beta V(x)} d x & =\int_{-\infty}^{0} x e^{-\beta x^{2}}\left(1-\beta Q x^{4}\right) d x+\int_{0}^{\infty} x e^{-\beta x^{2}} d x \\
& =-\beta Q \int_{0}^{\infty} x^{5} e^{-\beta x^{2}} d x \\
& =(-\beta Q)\left(-\beta^{-3}\right) \\
& =Q \beta^{-2}
\end{aligned}
$$

And for the denominator in (.2):

$$
\begin{aligned}
\int_{-\infty}^{\infty} e^{-\beta V(x)} d x & =\int_{-\infty}^{0} e^{-\beta x^{2}} d x+\int_{0}^{\infty} e^{-\beta x^{2}}\left(1-\beta Q x^{4}\right) d x \\
& \approx\left(\frac{\pi}{\beta}\right)^{1 / 2}
\end{aligned}
$$

In total we get for the mean excitation:

$$
\begin{aligned}
& \langle x\rangle=\frac{Q \beta^{-2}}{\left(\frac{\pi}{\beta}\right)^{1 / 2}} \\
& \langle x\rangle \sim Q T^{\frac{3}{2}}
\end{aligned}
$$

Which explains the temperature dependence with the characteristic exponent 1.5 in Figure .2, taking the mean excitation of the oscillator as the mean expansion of the whole system, i.e. $\langle x\rangle \sim\langle A\rangle$. 


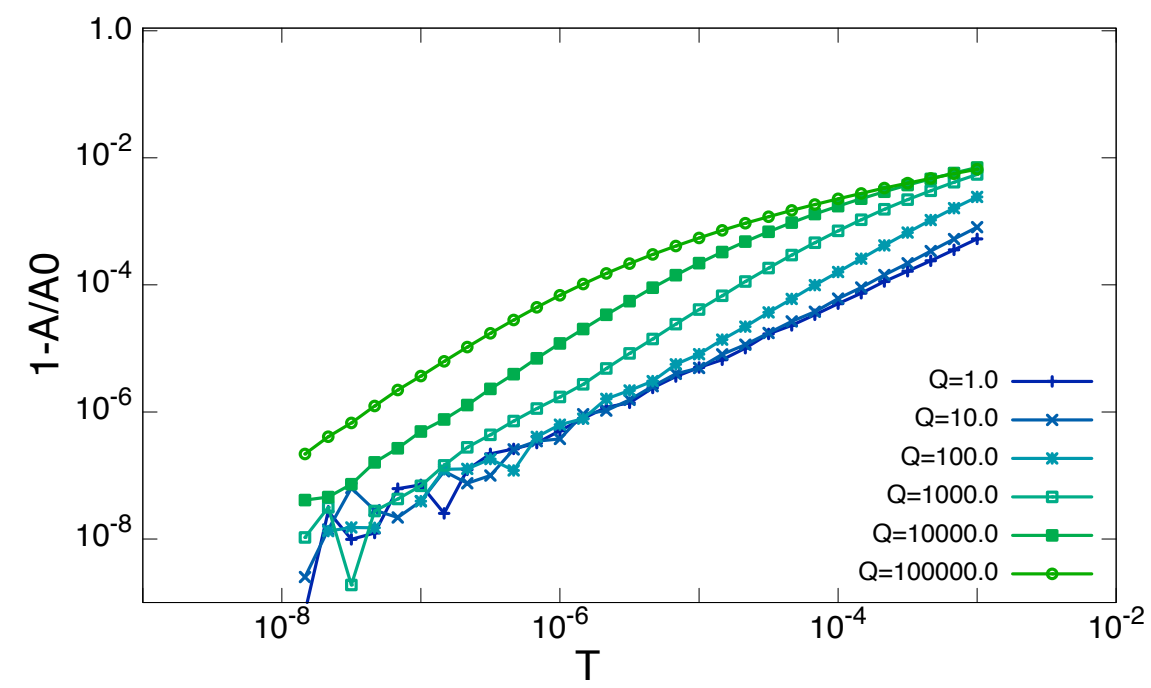

Figure .3: Quartic attraction.

\section{Anharmonic Interactions - quartic attraction}

$$
V(d)= \begin{cases}\left(d-d_{0}\right)^{2}, & \text { if } d \leq d_{0} . \\ \left(d-d_{0}\right)^{2}+Q\left(d-d_{0}\right)^{4}, & \text { if } d \geq d_{0} .\end{cases}
$$

\section{Double Quartic}

We use:

$$
V(d)= \begin{cases}Q\left(d-d_{0}\right)^{4}, & \text { if } d \leq d_{0} \\ Q\left(d-d_{0}\right)^{4}, & \text { if } d \geq d_{0} .\end{cases}
$$

In the case of the double quartic potential the NTE effect remains for $Q$ up to at least $10^{5}$ and the full range of relevant temperatures $T<10^{-3}$. 


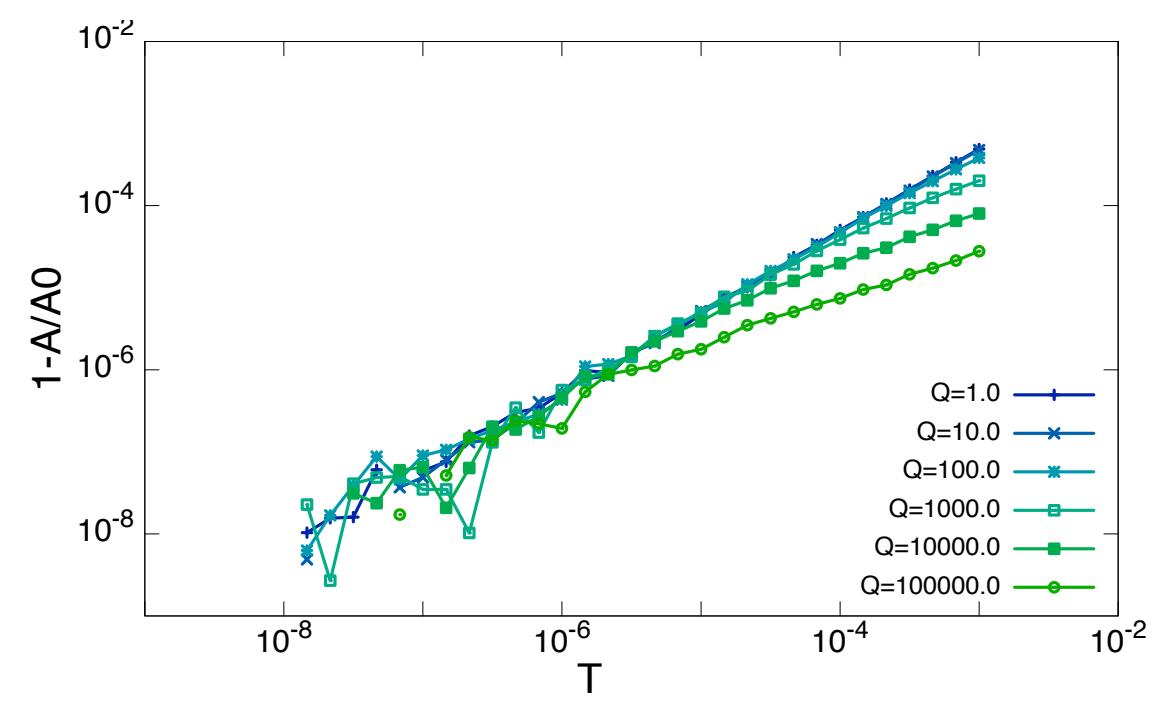

Figure .4: Double quartic potential. 


\section{Acknowledgements}

First and foremost I would like to thank Oskar Hallatschek. I am grateful that I had the opportunity to work with him on this intriguing project. The work with him was surely formative in the best sense. I have learned a lot about how to approach scientific problems. Oskar never ran out of ideas how to solve problems of all sorts whenever they occurred on this journey. He was always supportive to let me consider new paths in the project.

To no lesser extent do I want to thank Claus Heussinger. His support was fundamental for this project. The practical hints he gave were invaluable and his help was reliable at - almost literally - all times. His ideas in all aspects of the project shaped this work significantly.

I thank Florentin Wörgotter - and his great group - for being a safe haven for questions of all sorts. In every situation I could be sure of his full support and honest opinion. His experience and coolness are a great example to me.

I am most grateful to the group Biological Physics and Evolutionary Dynamics of the Max Planck Institute for Dynamics and Self-Organization for the great atmosphere at all times. Fabian, Sven, Jörn, Hedvika, Jean-François - I would like to thank them especially for being great colleagues. The same holds for Bernhard Altaner and Marco Mazza of the Dynamics of Complex Fluids department.

My special thanks go to Lukas Geyrhofer and Jens Nullmeier. They were much more than great colleagues and intellectually challenging folks at discussions on all sorts of topics. I wish and am certain that we will continue our paths as good friends.

My gratitude belongs to Barbara Kutz. Without her, Bunsenstraße would certainly have jammed at some point.

I thank the IMPRS-PBCS for financial support and Antje Erdmann for an open ear on any issue and the best administrational support imaginable. Antje's work is fundamental to providing great and fair conditions for $\mathrm{PhD}$ candidates at our graduate school.

I am most grateful to Danny Fliegner, ull and fulla. In hindsight I realize how smooth computing on the clusters was, and this thesis would have been impossible without such reliable infrastructure. 
The developers of great open source software and tools which we take for granted should be mentioned as well. I thank those who develop: gsl, python, vim, macvim, gcc, sqlite3, lammps, gnuplot, homebrew, latex, bibDesk, Skim.

I thank Carl Schwendinger-Schreck for great discussions and encouraging feedback on large parts of this work - equally, Carolina Brito.

I thank my friend Leo for being the best moral support possible for the whole duration of this work. The exact same goes for Andreas Volkhardt.

My parents Gertrude and Jan deserve their own chapter of acknowledgements. Their support on every level was beyond all question at any single point in time. The way they keep calm when things become tough at times and their unconditional love for our family are unrivalled.

I thank my brothers Eike and Sebastian and sisters-in-law Michal and Stefanie for being always highly supportive and their children for most-welcome distractions.

My teachers Hartmut Volkert (Mathematics), Rolf Engler (Physics), Bernhard Ziegler (Biology) I thank for sparking and supporting my interest in sciences and Wolfgang Nolting (Humboldt-Universität zu Berlin), Bernhard Mehlig (Göteborgs Universitet) and Eytan Domany (Weizmann Institute of Science) for being inspiring scientists and great mentors on my path through my university studies of complex systems.

More is different. 